Streptokinase resistance is present two years after initial administration of streptokinase

M B Buchalter, G Suntharalingham, I Jennings, C Hart, R J Luddington, R K Chaveraverty, P L Weissberg, T P Baglin

Department of Medicine, University of Cambridge; and Department of Haematology, Addenbrooke's Hospital, Cambridge

Thrombolytic treatment is an accepted therapy for acute myocardial infarction and streptokinase (SK) is the most commonly used agent in the United Kingdom; however, SK is antigenic and causes antibody formation that may affect the activity of a subsequently administered dose. To measure antibody mediated resistance to $S K$ in the general population and after exposure to SK or streptococcal infection, venous blood samples were taken from 30 normal people, 40 patients admitted to a coronary care unit before and on days 4 and 10 after receiving SK, 12 patients one year after receiving $S K, 12$ patients two years after receiving $S K$, and 12 patients after proven streptococcal infection. Streptokinase resistance was measured by a dilution neutralisation assay (Neut, $625 \mathrm{u} / \mathrm{ml}$ being the concentration to neutralise 1.5 million units of SK), an enzyme linked immunosorbent assay (ELISA) to measure total IgG anti-SK antibodies and a fibrin plate lysis system (FPL, in vitro clot lysis in the presence of patients' serum, $100 \%$ lysis meaning no inhibition by the patients serum, $0 \%$ meaning complete inhibition). Anti-SK activity (mean (SD) for normal people was Neut 87 (10) u/ml, ELISA 28 (6.3) $\mathrm{u} / \mathrm{ml}$, and FPL 100 (4)\% which were not different from day 0 values for the coronary care unit patients. In the coronary care unit patients anti-SK activity on day $\mathbf{0 \text { , day }}$ 4 , and day 10 was $68(6 \cdot 1) \mathrm{u} / \mathrm{ml}, 375(127)^{\star} \mathrm{u} / \mathrm{ml}$, and 3667 $(299)^{\star} \mathrm{u} / \mathrm{ml}$ for the Neut assay, $18(4.5) \mathrm{u} / \mathrm{ml}, 38(30 \cdot 7)$ $\mathrm{u} / \mathrm{ml}$, and $311(133)^{\star} \mathrm{u} / \mathrm{ml}$ for the ELISA, and $100(9) \%$, $94(8) \%$ and $17(14) \%$ * for the FPL (*indicates $\mathrm{p}<0.01 v$ day 0 ). Anti-SK activity was significantly raised (increased ELISA and reduced FPL values) in patients tested at one year (ELISA $120(25) \mathrm{u} / \mathrm{ml}^{\star}$ and FPL $43(9 \cdot 7) \%{ }^{\star}$ ), at two years (ELISA $198(44) \mathrm{u} / \mathrm{ml}^{\star}$ and FPL $31(19) \% \star$ ), and after streptococcal infection (ELISA $177(44)^{\star} \mathrm{u} / \mathrm{ml}$ and FPL $50(10) \%$ (*indicates $\mathrm{p}<0.01 v$ normal controls). At both one and two years after SK administration $66 \%$ of patients had sufficient anti-SK activity to neutralise completely a standard dose of SK.

Thus whereas anti-SK activity is low in the population receiving SK for the first time, this activity develops early after treatment and is present in most patients for up to two years after the initial dose. Readministration of SK may be ineffective for at least two years.

Frusemide-induced natriuresis is augmented by ultra-low-dose captopril but not by standard doses of captopril in chronic heart failure

J G Motwani, M K Fenwick, J J Morton, A D Struthers

Departments of Clinical Pharmacology and Medical Physics, Ninewells Hospital and Medical School, Dundee; and MRC Blood Pressure Unit, Western Infirmary, Glasgow

We have studied whether a very low starting dose ( $1 \mathrm{mg})$ of the angiotensin converting enzyme inhibitor captopril might better preserve the natriuretic response to the loop diuretic frusemide in chronic heart failure than the conventional higher dose of $25 \mathrm{mg}$ captopril, which tends to attenuate this response acutely. Ten patients with New York Heart Association grade II-III chronic heart failure and left ventricular ejection fractions of 24 to $42 \%$ were each treated on three occasions with oral frusemide plus placebo, $1 \mathrm{mg}$ captopril, or $25 \mathrm{mg}$ captopril in randomised double blind crossover fashion. As compared with placebo, $1 \mathrm{mg}$ captopril caused only slight non-significant falls in mean arterial pressure and circulating angiotensin II (AII) concentration and had no effect on glomerular filtration rate, estimated as the elimination of ${ }^{51} \mathrm{Cr}$-EDTA. By contrast, $25 \mathrm{mg}$ captopril produced a considerable fall in mean arterial pressure $(p<0.0001)$, obvious suppression of serum AII concentration ( $p<0.002)$, and a fall in glomerular filtration rate ( $p=0.0007)$. Whereas $25 \mathrm{mg}$ captopril caused moderate attenuation of frusemide induced natriuresis, $1 \mathrm{mg}$ captopril augmented the natriuretic response to frusemide by $104 \%$ ( $<<0.05 v$ placebo). In the face of frusemide induced renal arteriolar dilatation in chronic heart failure, captopril at a starting dose of $1 \mathrm{mg}$ (but not $25 \mathrm{mg}$ ) preserves enough circulating AII to maintain efferent arteriolar tone and thus glomerular filtration, while augmenting the renal tubular natriuretic response to frusemide.

In a subgroup of chronic heart failure patients with intractable fluid retention, use of ultralow dose captopril, rather than conventional doses, may more effectively facilitate the natriuretic response to frusemide.

\section{Response to candoxatrilat in patients with severe heart failure}

J Good, S Watson, M Ghalei, N Jackson, M Peters, G Frost, C Oakley, J Cleland

Department of Medicine (Cardiology), Royal

Postgraduate Medical School, Hammersmith Hospital, London

Candoxatrilat, a neutral endopeptidase inhibitor, has beneficial actions in patients with mild heart failure but there have been few studies in severe heart failure. Intravenous boluses of endoxatrilat $(50 \mathrm{mg}, 100 \mathrm{mg}$, and 200 $\mathrm{mg}$ ) and placebo were given on separate days to 12 patients with severe heart failure (ejection fraction $36(2) \%$ ) using a blinded four way crossover design. Blood pressure and urine output were measured two hourly for eight hours after each bolus. Renal blood flow and glomerular filtration rate were measured radioisotopically. Plasma hormones were measured before and three hours after dosing. There was no difference in response between the candoxatrilat doses tested. Compared with placebo, $100 \mathrm{mg}$ candoxatrilat increased urine flow (56 (11) $v 143(24) \mathrm{ml} / \mathrm{h}$, $\mathrm{p}<0.01)$ and sodium output $(3.6(1.2) v 7.6(1.5) \mathrm{mmol} / \mathrm{h}$, $\mathrm{p}<0.01)$. Diuresis was greatest up to four hours after the dose although effects persisted for at least six hours. Candoxatrilat increased urinary magnesium $(p<0.01)$ and cyclic guanosine monophosphate concentrations ( $\mathrm{p}<0.001$ ) over eight hours but not potassium. Plasma aldosterone concentration fell (178 (41) v $103(26) \mathrm{pg} / \mathrm{ml}$, $\mathrm{p}<0.01$ ) but vasoactive intestinal polypeptide concentration increased $(p<0.05)$. Overall there were small increases in glomerular filtration rate and renal blood flow, and a fall in blood pressure (NS). In four patients, urine flow increased $<75 \%$ over the first four hours (mean $25 \%$, range -17 to $73 \%$ ) with a fall in glomerular filtration rate ( 76 to $64 \mathrm{ml} / \mathrm{min}$ ). In eight patients urine flow increased 
$>90^{\circ}{ }_{0}$ over the first four hours with an increase in glomerular filtration rate $(70(11)$ to $99(9) \mathrm{ml} / \mathrm{min}, \mathrm{p}<0.05)$.

Unlike many reports with infusions of atrial natriuretic peptide, candoxatrilat causes diuresis in patients with severe heart failure.

\section{Effect of sumatriptan (GR43175) on central haemodynamics and coronary circulation}

\author{
P D MacIntyre, J D Gemmill, K J Hogg, B Bhargava, \\ W S Hillis \\ University of Glasgow
}

Sumatriptan, a selective $5 \mathrm{HT}_{1}$-like agonist is effective in the acute treatment of migraine. Its effects on coronary artery dimensions and central haemodynamics were assessed in 10 patients (six men, four women, age 52.9 (9.8)) undergoing diagnostic coronary angiography. Mean systemic arterial pressure, mean pulmonary artery pressure, hęart rate, and 12 lead electrocardiogram were recorded at five min intervals during placebo infusion followed by a $10 \mathrm{~min}$ infusion of sumatriptan to a total of 48 $\mu \mathrm{g} / \mathrm{kg}$. Coronary artery dimensions were measured using digital calipers at identified segments on hard copy end diastolic frames obtained with digital subtraction angiography at baseline, and after each infusion. There were no changes in heart rate or electrocardiographic morphology with either infusion. Baseline measurements (mean (1 SD)) were mean systemic arterial pressure $100(13 \cdot 2) \mathrm{mm} \mathrm{Hg}$, mean pulmonary artery pressure $15.2(6.3) \mathrm{mm} \mathrm{Hg}$, and mean coronary artery diameter $4.3(1.6) \mathrm{mm}$. No significant change followed placebo infusion: mean systemic arterial pressure $99(11 \cdot 6) \mathrm{mm} \mathrm{Hg}$, mean pulmonary artery pressure $16.2(6.2) \mathrm{mm} \mathrm{Hg}$, mean coronary artery diameter $4.2(1.6) \mathrm{mm}$. Sumatriptan caused a vasoconstrictive response with increase in mean systemic arterial pressure to $115(22.5) \mathrm{mm} \mathrm{Hg}(\mathrm{p}<0.001)$, mean pulmonary artery pressure to $25.5(11.2) \mathrm{mm} \mathrm{Hg}$ $(\mathrm{p}<0.001)$ and a reduction in coronary artery diameter to $3.6(1.6) \mathrm{mm}(\mathrm{p}<0.001)$ The mean percentage reduction from baseline in mean coronary artery diameter was 13.9 (9.6) $\%$ (confidence interval 10.9 to 16.9 ).

Sumatriptan, a selective $5 \mathrm{HT}_{1}$ agonist causes a vasopressor response in both pulmonary and systemic circulations with, in addition, coronary artery vasoconstriction.

\section{Autonomic reflexes stimulated by coronary balloon angioplasty: regional differences in afferent population}

\section{F Shakespeare, A Crowther, I C Cooper, D J Coltart, M M Webb-Peploe \\ Cardiac Department, St Thomas's Hospital, London}

The sensory innervation of the heart is thought to be autonomically mediated although direct stimulation of the afferents has been poorly documented in humans. The aim of the study was to stimulate the afferents directly in patients undergoing elective percutaneous transluminal coronary balloon angioplasty (PTCA) and record efferent responses. Efferent responses measured by changes in heart rate, mean arterial pressure, plethysmographic forearm blood flow and resistance were compared in groups during balloon inflation of the left anterior descending artery (LAD), right coronary artery (RCA), and circumflex artery (CX). All balloon inflations were for at least $30 \mathrm{sec}-$ onds and the maximal change recorded in that time. Ten male patients underwent PTCA to 10 lesions in the LAD artery, nine patients to 10 lesions in the RCA, and five patients to five lesions in the CX. The heart rate increased by $12 \cdot 1(5 \cdot 2) \%$ (mean (SEM)) in the LAD group and decreased by $6.1(3.0) \%$ in the RCA group ( $p<0.04)$. It did not change in the CX group. Mean arterial pressure increased by $11.7(2.9) \%$ in the LAD group and decreased by $1.2(1.9) \%$ in the RCA group (p < 0.002). In the CX group, mean arterial pressure decreased by $0.9(2.9) \%$, which was significantly different from the LAD group $(\mathrm{p}<0.02)$. Forearm blood flow decreased by $34.3(8 \cdot 8) \%$ in the LAD group and increased by $66.8(33.0) \%$ in the RCA group ( $<<0.01$ ). In the CX group, it increased by $49 \cdot 3(21.7) \%$, which was significantly different from the LAD group. Forearm resistance increased by $86.3(13.5) \%$ in the LAD group and decreased $11.3(26.8) \%$ in the RCA group ( $\mathrm{p}<0.005)$. In the CX group, it decreased by 22.7 $(17 \cdot 6) \%$, which was significantly different from the LAD group ( $\mathrm{p}<0.0004)$.

These data indicate that PTCA induces a reflex initiated by the heart. Angioplasty stimulation in the RCA and CX territory resulted in a vagally mediated reflex, and in the LAD territory, a sympathetically mediated reflex. This supports findings from animal studies that the inferior surface of the left ventricle has a predominantly vagal afferent innervation and the anterior surface a predomiantly sympathetic afferent innervation.

\section{Comparison of angiography and intravascular ultrasound in the detection of early atherosclerosis

\author{
A N S Deaner, A Cubukcu, P J Scott, A R Essop, \\ G J Williams \\ Killingbeck Hospital, Leeds
}

The presence of arterial disease is recognised by angiography which relies on the visualisation of luminal narrowing but poorly differentiates the cause of narrowing, that is, thrombus, embolisation, spasm, or atheroma. In the absence of luminal narrowing an artery is usually assumed to be disease free. Our early experience with intravascular ultrasound suggested that we could detect changes in the intima and media of arteries in vessels that were angiographically normal. In vitro work has confirmed that atheroma can be clearly seen with intravascular ultrasound and that even the earliest changes of atherosclerosis produce a typical thickening of the intima. We have carried out in vivo studies of peripheral arteries in 15 patients to assess the sensitivity of intravascular ultrasound compared with angiography. We compared the findings of digital subtraction angiography with intravascular ultrasound in 29 segments of femoral and iliac artery. A haemostatic sheath was placed in the femoral artery. A 6 french intravascular ultrasound catheter $(20 \mathrm{MHz})$ was inserted through the sheath and positioned at the iliac bifurcation. A radioopaque ruler was placed at the level of the artery. A digital subtraction angiogram was taken using a hand injection of $10 \mathrm{ml}$ of non-ionic contrast. The catheter was withdrawn at $2 \mathrm{~mm}$ intervals. Sequential images were recorded on video tape. The arteries were divided into $5 \mathrm{~mm}$ segments and the presence of stenosis or vessel wall irregularity noted from the angiogram and compared with the findings of the intravascular ultrasound images taken at the same positions within the artery. The images were regarded as abnormal 
if intimal thickening, plaque, or calcification were present. Of 29 segments studied 11 were angiographically abnormal. Twenty three segments were abnormal when studied by intravascular ultrasound including all the angiographically abnormal segments. Only $48 \%$ of abnormal segments as shown on intravascular ultrasound were thus detected by angiography.

Intravascular ultrasound is able to show atherosclerotic changes in angiographically normal arteries. Not only were very early intimal changes visualised by intravascular ultrasound but quite mature sizable plaques were frequently missed by angiography because of compensatory atrophy of the media underlying the plaque. Luminal encroachment occurs late in the development of atheromatous arterial disease, a fact that should be taken into account particularly in studies of atheroma regression.

\section{Doppler derived transmitral velocity indices: changes occurring during alteration of ventricular preload}

C M Bellamy, P A Dodds, E D Grech, K F Robotham, M A Fox, B M Fabri, R A Perry

The Cardiothoracic Centre, Liverpool

It has been postulated that Doppler derived transmitral blood velocity indices reflect changes in left ventricular diastolic function. The relation, however, between left ventricular filling pressure, diastolic function, and transmitral Doppler indices remains uncertain. To examine this relation we studied 22 patients undergoing coronary artery bypass surgery and who had left ventricular ejection fractions $>40 \%$. Transmitral blood velocity was measured at varying filling pressures by transoesophageal echocardiography after opening of the pericardium and before initiation of cardiopulmonary bypass. Transmitral velocities were recorded in a basal state and after fluid loading. The maximum early diastolic filling velocity (Emax); the maximum velocity at atrial contraction (Amax); the E/A velocity ratio; the $\mathrm{E}$ wave maximum velocity/total diastolic time velocity integral (E/TVI); the mean acceleration slope ( $\mathrm{E}$ accslope) and time ( $\mathrm{E}$ acctime) to maximum $\mathrm{E}$ wave velocity; and the mean deceleration slope (E decslope) and time ( $E$ dectime) from peak $E$ wave velocity linearly extrapolated to base line were recorded in both loading conditions. The total increase in pulmonary capillary wedge pressure was from $5.6 \mathrm{~mm} \mathrm{Hg} \mathrm{(95 \%} \mathrm{CI} 4.5$ to 6.7$)$ to 10.0 $\mathrm{mm} \mathrm{Hg} \mathrm{(8.7} \mathrm{to} \mathrm{11.4)} \mathrm{(p} \mathrm{<} \mathrm{0.0005).} \mathrm{Emax} \mathrm{increased} \mathrm{from}$ $39.5 \mathrm{~cm} / \mathrm{s}$ to $59.5 \mathrm{~cm} / \mathrm{s}(\mathrm{p}<0.0001)$; Amax increased from $56.1 \mathrm{~cm} / \mathrm{s}$ to $67.9 \mathrm{~cm} / \mathrm{s}(\mathrm{p}<0.0001)$; the $\mathrm{E} / \mathrm{A}$ ratio increased from 0.73 to $0.91(\mathrm{p}<0.0001)$; $E$ accslope increased from $502 \mathrm{~cm} / \mathrm{s}^{2}$ to $640 \mathrm{~cm} / \mathrm{s}^{2}(\mathrm{p}<0.001)$; E acctime increased from $89.9 \mathrm{~ms}$ to $100.5 \mathrm{~ms}$ ( $\mathrm{p}<0.025$ ); and E desclope increased from $-186 \mathrm{~cm} / \mathrm{s}^{2}$ to $-279 \mathrm{~cm} / \mathrm{s}^{2}$ ( $p<0.003) ; \mathrm{E}$ dectime and E/TVI showed no significant change.

Changes in left ventricular filling pressure can significantly alter Doppler derived transmitral velocity indices and therefore this needs to be taken into account when trying to relate transmitral blood flow and diastolic function.

\section{Ventricular tachyarrhythmias after myocardial} infarction: what does surgery offer?

\section{J P Bourke, C J Hilton, J M McComb, S S Furniss,} J C Doig, R W F Campbell

Cardiothoracic Unit, Freeman Hospital, Newcastle upon Tyne

Abolition of arrhythmia is the ultimate goal of treatment in patients with recurrent ventricular tachyarrhythmias after myocardial infarction. Whereas map guided endocardial resection can achieve this, it remains underused because many view the overall risk of surgery for these patients as unacceptably high. The aim of this analysis was to define the risk of endocardial resection for clinically recognisable patient subgroups by relating outcome to the clinical context in which surgery was performed. In the period 1980-91 endocardial resection was offered to all patients referred to this unit with life threatening drug refractory ventricular tachyarrhythmias regardless of age, time from acute myocardial infarction, or ventricular function. One hundred patients underwent endocardial resection, which was conducted in a uniform way throughout the study period. Mean age of patients was 56 (10) years, 81 were men and the myocardial infarction was anterior in 71 . Mean left ventricular ejection fraction was $28 \%$ (range 5 to $50 \%$ ) and 60 had a left ventricular aneurysm. The mean number of major ventricular tachyarrhythmic episodes per patient was 11 (range 2 to 200) and drug failures 4 (2). Emergency surgery was required for intractable ventricular tachyarrhythmias in 28 patients, and 32 of the total had surgery within eight weeks ("early") of acute myocardial infarction. Endocardial resection was performed in all. In addition aneurysmectomy was performed in 57, cryoablation of lesions in 26, and antiarrhythmic ventriculotomies in 11 . Twenty five patients died within 30 days of surgery, 21 in low output cardiac failure. There have been no postoperative deaths from arrhythmias and only six patients required antiarrhythmic drug treatment. Outcome within 30 days of endocardial resection was critically related to the clinical context in which surgery was undertaken. Thus mortality for elective endocardial resection more than eight weeks after myocardial infarction, "early" post-myocardial infarction, emergency endocardial resection, and "early" emergency were $18 \%, 34 \%, 46 \%$, and $56 \%$ respectively. The high overall mortality reflects the type of patients undergoing endocardial resection at this unit. Actuarial survival rates for the total group at one, three, and five years after surgery were $70 \%, 68 \%$, and $66 \%$ respectively.

Surgery offers a cure of ventricular tachyarrhythmias at a risk proportional to the patient's preoperative risk of sudden death. It has a unique role in patients with intractable ventricular tachyarrhymia and has yet to achieve its full potential.

\section{Discrimination of ventricular tachycardia from exercise induced sinus tachycardia by pulsatile right ventricular pressure monitoring}

S C Heald, Y Bashir, V E Paul, N J Randall, K A Collins, D E Ward, A J Camm

St George's Hospital, London

The increasing complexity of implantable cardioverter defibrillators (ICDs) with the introduction of tiered treat- 
ment devices has expanded their application to include patients with slower pace terminable arrhythmias; however, rate criteria alone are not always sufficient to differentiate physiological tachycardia from haemodynamically stable ventricular tachycardia (VT). We have studied the use of right ventricular pulse pressure (RVPP) monitoring in distinguishing exercise induced sinus tachycardia from VT. The RVPP was recorded from 11 patients with recurrent VT (age 35-70; left ventricular ejection fraction $20-65 \%$, coronary heart disease 8/11) during treadmill exercise testing and induced VT. Pressure signals were obtained via a percutaneous micromanometer catheter (Telectronics Pacing Systems), digitised at $1 \mathrm{kHz}$ with a bandwidth of dc-250 Hz, and stored on optical disc before analysis. The RVPP and maximum $\mathrm{dP} / \mathrm{dt}(\mathrm{dP} / \mathrm{dt}$ max) during peak exercise and sustained haemodynamically stable VT were expressed as a percentage of resting sinus rhythm values. Peak heart rate during exercise ranged from 78 to 187 beats/min. Heart rate during VT ranged from 139 to $225 \mathrm{bpm}$. Mean RVPP fell to a greater extent than $\mathrm{dP} / \mathrm{dt}$ max during induced VT (to $63(33)^{\circ}{ }_{0} v 83(49)^{\circ}{ }_{0}$ ) but both increased greatly during exercise $\left(176(46)^{\circ}{ }_{0} v 234\left(79^{\circ}{ }_{0}\right)\right.$. With a $30 \%$ fall compared with baseline sinus rhythm as a cut-off, RVPP diagnosed VT correctly in $9 / 11$ patients (sensitivity $82 \%$, specificity $100^{\circ}{ }_{0}$ ) whereas $\mathrm{dP} / \mathrm{dt} \max$ only diagnosed VT correctly in $4 / 11$ patients (sensitivity $36 \%$, specificity $100 \%$ ).

Preliminary results suggest that VT may be discriminated from physiological tachycardia with a high degree of specificity using measurement of RVPP and $\mathrm{dP} / \mathrm{dt} \max$, but that the greater sensitivity of RVPP may be more suitable for clinical application. In the future, ICDs incorporating RVPP monitoring may allow increased use of antitachycardia pacing where previously distinction between VT and sinus tachycardia was not possible.

\section{Fractionation of paced ventricular electrograms in hypertrophic cardiomyopathy is associated with an increased risk of sudden death}

\author{
R C Saumarez, A Panagos, $M$ de Belder, I Simpson, \\ J Stewart, W McKenna, A Camm \\ St George's Hospital Medical School, London
}

Myofibrillar disarray in hypertrophic cardiomyopathy may cause dispersion of activation throughout the myocardium and this dispersion may form the substrate of ventricular fibrillation. Variations in myocyte dimensions may cause differences in conduction velocities and refractory periods and myocyte disarray may provide a number of conduction paths between two sites that may be recruited or blocked according to the refractory state of the intervening tissue. This hypothesis was tested in 37 patients with hypertrophic cardiomyopathy, of whom four had out of hospital ventricular fibrillation, five had ventricular tachycardia on ambulatory monitoring, 15 had a family history of sudden death, and four controls were studied by pacing one site in the right ventricle and recording electrograms from three other right ventricular sites. Intraventricular conduction curves were obtained with S1S2 coupling intervals decreasing in $1 \mathrm{~ms}$ steps from $479 \mathrm{~ms}$ to ventricular effective refractory period. These curves were repeated by pacing each right ventricular site in turn. The electrograms were high pass filtered to emphasise fractionation and two parameters: the point at which latency increased by $0.75 \mathrm{~ms}$ over a change of $20 \mathrm{~ms}$ in the S1S2 coupling interval and the increase in electrogram width between a coupling interval of $350 \mathrm{~ms}$ and ventricular effective refractory period were extracted. Linear discriminant analysis showed that patients with ventricular fibrillation, ventricular tachycardia, or family history of sudden death had increased electrogram width and early increase in latency and form a group that was distinct from low risk patients and controls $(p<0.0000001)$. Patients with ventricular fibrillation had the most pronounced electrogram widening and early increase in latency, and fell into a distinct group compared with the remaining patients $(p<0.0001)$.

This novel technique shows a consistent electrophysiological abnormality in hypertrophic cardiomyopathy and warrants prospective testing as a predictor of the risk of sudden death.

\section{Inhibition in the human heart by long duration} subthreshold pulses: spatial and temporal effects

\section{T Connelly, A D Cunningham, E Rowland}

Royal Brompton National Heart and Lung Hospital, London

Termination of tachycardias by non-propagated stimuli has been reported, but the mechanism of this effect is not clear. We have shown that long duration subthreshold cathodal constant current pulses can produce large degrees of inhibition in the human heart, and this might be a possible mechanism for termination of tachycardia. We investigated further the effect of inhibition in 12 patients undergoing electrophysiological studies. Constant ventricular pacing ( $\mathrm{S} 1 \mathrm{~S} 1=600 \mathrm{~ms}$ ) was performed, and effective refractory period was determined with a premature beat (S2) with an amplitude of twice the late diastolic threshold. The S1S2 interval was set at effective refractory period + $10 \mathrm{~ms}$, and a cathodal conditioning pulse ( $\mathrm{Sc}$, duration = S1S2) was introduced, beginning synchronous with $S 1$, and increased in amplitude until $S 2$ failed to capture. The duration of Sc was then reduced in steps of 5 to $10 \mathrm{~ms}$, leaving a gap between $S c$ and $S 2$. Inhibition was shown to occur across gaps of up to 50 ms (mean $39 \mathrm{~ms}$ ) with Sc amplitudes below late diastolic threshold in twelve patients; with further increases in Sc amplitude, inhibition could be produced more than $100 \mathrm{~ms}$ beyond the end of Sc. In a further eight patients we investigated whether an inhibitory effect could be produced at an adjacent pole on the same catheter; $S 1$ and S2 were delivered as already described, and Sc (duration 10 to $20 \%$ longer than S1S2) was introduced at another point 1 to $5 \mathrm{~mm}$ away. Subthreshold values of Sc did not inhibit capture by S2; even when Sc amplitude was increased up to five times late diastolic threshold, no inhibition could be produced at a point $1 \mathrm{~mm}$ away.

These studies suggest that termination of tachycardia by subthreshold pulses is only feasible with a catheter positioned very close to a critical point on a re-entrant circuit. Termination of tachycardia by a non-propagated impulse is more likely to occur by local capture. 


\section{Relation between QT intervals and epicardial monophasic action potential}

\author{
P D Higham, C J Hilton, C J Griffiths, S S Furniss, \\ R W F Campbell \\ University Department of Cardiology, Freeman \\ Hospital, Newcastle upon Tyne
}

To test the hypothesis that QT dispersion (QT max minus QT min) measures risk of arrhythmia by reflecting dispersion of ventricular recovery at the body surface monophasic action potential, studies were performed in eight patients undergoing routine cardiac surgery.

Monophasic action potentials were recorded from 10 left and two right ventricular sites with a reference electrode used to measure activation. Recordings were made in sinus rhythm and then with left ventricular pacing (to increase dispersion of ventricular recovery). Simultaneous 12 lead electrocardiograms were recorded at $50 \mathrm{~mm} / \mathrm{s}$. Mean dispersion of ventricular recovery time (activation time plus repolarisation time) in sinus rhythm was 53 (12) $\mathrm{ms}$ and increased to $86(26) \mathrm{ms}(\mathrm{p}=0.01)$ with pacing. Mean QT dispersion in sinus rhythm was 43 (10) $\mathrm{ms}$ and also increased on pacing to $92(27) \mathrm{ms}(\mathrm{p}=0.001)$. There was a positive correlation between the change in dispersion of ventricular recovery time produced by pacing and the change in QT dispersion (Spearman's $\rho=0.76, \mathrm{p}=0.02$ ). Dispersion of ventricular recovery by pacing was almost entirely due to increased dispersion of activation time (mean increase $=46(26) \mathrm{ms}, \mathrm{p}=0.001$ ).

This study correlates body surface electrocardiographic QT measurements with perioperative monophasic action potentials in humans and shows a close relation between the two. Changes in QT dispersion induced by ventricular pacing correlate well with changes in the dispersion of ventricular recovery. As hypothesised QT dispersion does reflect dispersion of ventricular recovery.

\section{Dobutamine is the most physiological stress for echocardiographic assessment of regional wall motion in multivessel coronary artery disease}

\author{
C A MacRae, I A Simpson, S Gibson, J F Sneddon, \\ A J Camm \\ St George's Hospital, London
}

Stress echocardiography is being evaluated for the detection of reversible wall motion abnormalities in ischaemic heart disease before and after revascularisation. To assess the degree to which a variety of stresses reproduce the wall motion abnormalities of physiological exercise we studied a group of patients with resting left bundle branch block and angiographically proven multivessel coronary artery disease in whom the target lesion could not be determined by conventional angiographic or exercise electrocardiographic criteria. All patients underwent echocardiography during maximal treadmill exercise, incremental atrial pacing, infusion with adenosine $(0 \cdot 15$ $\mathrm{mg} / \mathrm{Kg}$ ), high dose dipyridamole $(0.84 \mathrm{mg} / \mathrm{Kg})$, and incremental doses of dobutamine to maximal predicted heart rate or ischaemic endpoints. All pharmacological agents were well tolerated. Two subjects developed nonsustained ventricular tachycardia with dobutamine but both had histories of angina related ventricular tachycardia. There was no evidence of rate or ischaemia related changes in conduction. Overall regional wall motion indices were assessed on an established numerical grading of 1 to 4(1-normal, 2-hypokinetic, 3-akinetic, 4-dyskinetic) using a 21 segment system. Echocardiograms were analysed by two independent observers without knowledge of the stress mode or angiographic findings. Overall regional wall motion indices at rest were $1.87(0 \cdot 77)$ and increased significantly during exercise $(2 \cdot 22(0 \cdot 7))$ and dobutamine infusion $(2.28(0.75)) \quad(\mathrm{p}<0.05)$ but did not alter significantly with atrial pacing $(1.89(0.81))$ or dipyridamole infusion $(1.73(0 \cdot 65))$ and actually fell during adenosine infusion $(1.73(0.6))$ (NS). Stress induced regional wall motion abnormalities all corresponded to a region of myocardium subtended by a stenosed epicardial coronary artery and no individual segment score altered in a direction against the trend for a particular stress.

Only dobutamine produces equivalent physiological stress to treadmill exercise in patients with multivessel coronary artery disease as judged by overall regional wall motion indices and individual segment behaviour.

\section{Prognostic value of dobutamine echocardiography in patients with high pretest likelihood of} coronary artery disease

\section{P Mazeika, A Nadazdin, C M Oakley \\ Clinical Cardiology Unit, Hammersmith Hospital, London}

To investigate the usefulness of inducible regional asynergy on dobutamine stress echocardiography (DE) as a non-invasive predictor of future cardiac events. Fifty one symptomatic patients (age 54 (9), 45 men) with suspected coronary artery disease were studied using incremental doses up to $20 \mu \mathrm{g} / \mathrm{kg} / \mathrm{min}$. Pretest likelihood of coronary artery disease was $79 \cdot 7$ (SE 5.6)\% before, and $83.4(5 \cdot 2) \%$ after exercise electrocardiography with probability analysis based on age, sex, and symptoms. Cross sectional images were analysed by scoring an 11 segment model (normo or hyperkinesis $=1$, hypokinesis $=2$, akinesis $=$ 3 , dyskinesis $=4$ ) and gave good interobserver agreement $(\kappa=0 \cdot 77$ ). During 24 (4) months (range 19 to 32) of follow up 23 patients had cardiac events (one myocardial infarction, nine unstable angina, 10 coronary bypass surgery, three coronary angioplasty) and 28 were event free. Age, proportion with baseline asynergy (8/23 v6/28), and echocardiographic ejection fraction (modified Simpson's rule; measurable in 33) were similar in these 2 groups (all NS). A positive DE was seen in $17 / 23(74 \%$ ) with and $8 / 28(29 \%)$ without events ( $p=0.003,95 \%$ CI 14 to 69$)$; $5 / 6(83 \%)$ patients with involvement of three segments had events. Myocardial infarction or unstable angina occurred in $8 / 25(32 \%)$ with a positive, and $2 / 26(8 \%)$ with a negative $\mathrm{DE}(\mathrm{p}=0 \cdot 07)$. Concordance between exercise electrocardiography and DE was $73 \%$, and both exercise duration (389 (195) v $517(237) \mathrm{s}, \mathrm{p}<0.05)$ and time to diagnostic ST shift (291 (192) $v 447$ (212) s, p = 0.05) were shorter in those with inducible asynergy.

These data suggest that patients with probable coronary artery disease who have a positive $\mathrm{DE}$ are at higher risk of an adverse outcome. Thus DE may aid selection for coronary angiography and prognostic revascularisation, particularly in patients unable to exercise. 
Inotropic versus vasodilator agents for diagnostic stress echocardiography: a prospective study in 100 patients undergoing coronary arteriography

T Marwick, T Baudhuin, A M D'Hondt, J Melin, J M Detry

St Luc Hospital, University of Louvain, Brussels

Both inotropic and vasodilator agents have been combined with echocardiography for the diagnosis of coronary disease. The purpose of this study was to establish the optimal stress by comparing dobutamine echo (DE) and adenosine echo (AE), in the same patients and conditions. One hundred patients without evidence of previous infarction, undergoing coronary arteriography for clinical indications, were studied prospectively by $\mathrm{DE}$ and $\mathrm{AE}$ at the time of arteriography. Dobutamine was infused during clinical, electrocardiographic, and echocardiographic monitoring, in three-minute increments from five to $40 \mathrm{mcg} / \mathrm{kg} / \mathrm{min}$. Adenosine was infused under the same conditions, initially at $0.10 \mathrm{mg} / \mathrm{kg} / \mathrm{min}$, with three-minute stages at 0.14 and $0.18 \mathrm{mg} / \mathrm{kg} / \mathrm{min}$. In each case, the stress protocol was terminated at completion (peak dose, $>85 \%$ age predicted maximum heart rate, or appearance of severe ischemia), or the development of intolerable side effects. Digitised echo images before, during, and after stress were stored in a quad-screen, cine-loop display, and were evaluated qualitatively by independent observers. The DE protocol was completed in 68 patients and that for $\mathrm{AE}$ in 70 patients (NS). The commonest limitation for DE was hypotension $(n=15)$, whereas that for AE was dyspnoea $(n=14)$. All side effects were reversible and no serious complications occurred. The peak heart rate achieved at DE exceeded that at $\mathrm{AE}(111(24) v 92(15), \mathrm{p}<0.001)$ as did the increment in heart rate during the test $(20$ (5) $v 14$ (3) $\mathrm{p}<0.001)$, and the peak rate pressure product achieved $\left(42.7(20.1) v 21.6(10.9) \times 10^{3}, \mathrm{p}<0.001\right)$. In 62 patients with significant coronary disease (defined by the quantitation of $>50 \%$ stenosis in a major epicardial vessel), the sensitivity of DE was $84 \%$, and that of $\mathrm{AE}$ was $56 \%$ $(\mathrm{p}<0.001)$. This difference was more prominent in the 32 patients with single vessel disease $(81 \% v 50 \%, \mathrm{p}<0.01)$ than in those with multivessel disease $(87 \% v 63 \%$, $\mathrm{p}=0.07)$. The specificity of DE was $82 \%$, and that of AE was $92 \%$ (NS).

Thus DE has a significantly higher sensitivity than AE for the detection of coronary disease, and is the test of choice for pharmacological stress echo.

\section{A comparison of exercise echocardiography and technetium-99m MIBI SPECT in three dimensional images for identification of myocardial ischaemia}

\section{P A Joseph, K Robinson, P Crean, G Gearty, $M$ Walsh} Trinity Cardiology, St James' Hospital, Dublin

Exercise echocardiography is useful in the detection of myocardial ischaemia but has not been compared to technetium-99m metoxyisobutylisonitrile single photon computed tomography in three-dimensional images (SPECT). Fifty eight patients ( 37 men, 21 women; mean age 55.9 (9.4)) with recurrent episodes of angina of effort were studied. Abnormalities of left ventricular wall motion and thickness on exercise echocardiography and perfusion defects on SPECT suggesting myocardial ischaemia were compared with coronary angiography. The results obtained with both of these techniques were then compared with each other. The findings on exercise echocardiography correlated with those on coronary angiography in $52(89.7 \%$ ) of the 58 patients ( $<<0.0001$, $\kappa=0.817)$. Exercise echocardiography detected 42 $(87.5 \%)$ of 48 patients with significant ( $>75 \%$ stenosis) coronary artery disease in at least one vessel on coronary angiography. All of 10 patients with normal coronary angiograms had normal findings on exercise echocardiography (specificity $100 \%$ ). The findings of exercise SPECT correlated with those on coronary angiography in $35(60.3 \%)$ of 58 patients $(\mathrm{p}<0.0001, \kappa=0.344)$. Exercise SPECT detected $34(70 \%)$ of 48 patients with significant coronary artery disease. Ten out of 10 with normal coronary angiograms had normal exercise SPECT results (specificity $100 \%$ ). The concordance of exercise echocardiography with exercise SPECT in identifying myocardial ischaemia was $63.8 \%(p<0.0001, \kappa=0.384)$.

Exercise echocardiography is a highly sensitive and specific non-invasive technique useful in the evaluation of patients with ischaemic heart disease. Its greater sensitivity may render it a more useful investigation than SPECT in the detection of underlying coronary stenoses.

Simultaneous dipyridamole stress echocardiography and technetium-99m MIBI SPECT after uncomplicated infarction: comparison of prognostic values with exercise testing

A J McNeill, P M Fioretti, A Salustri, M M A Pozzoli, C C Broekema, A E M Reijs, E M El-Said, J R T C Roelandt

Thoraxcenter, Erasmus University, Rotterdam, The Netherlands

To compare the frequency of "ischaemia" seen during simultaneous dipyridamole echocardiography and technetium $99 \mathrm{~m}$ metoxyisobutylnitrile single photon emission computed tomography (SPECT), and compare their prognostic values with exercise electrocardiography, 90 patients with uncomplicated myocardial infarction underwent pre-discharge dipyridamole testing and those fit had maximal bicycle ergometry. Dipyridamole $(0.84 \mathrm{mg} / \mathrm{kg})$ was infused intravenously with continuous echo recording. Two minutes after dipyridamole, $370 \mathrm{MBq}$ Tc-99m MIBI was injected intravenously. Stress SPECT images were acquired one hour later and rest images acquired 24 hours after stress. SPECT reversible perfusion defects occurred in $49 / 90(54 \%)$ patients and echocardiographic new wall motion abnormalities in $31 / 88(35 \%)$ patients with assessable echoes $(\mathrm{p}<0.02)$. Of 66 patients fit for exercise testing, $33(50 \%)$ had ST depression and 28 had a negative test (no ST depression with achievement of $\geqslant 100 \mathrm{~W}$ workload). Eighty six patients were reviewed at a mean of 47 weeks after myocardial infarction; death had occurred in five of 30 with positive and one of 54 with negative dipyridamole echo $(p=0.02)$, in four of 47 with positive and two of 39 with negative SPECT $(p=0.43)$ and in none of the 25 patients with negative exercise tests.

After uncomplicated myocardial infarction, dipyridamole echocardiography or SPECT is a feasible alternative to exercise testing and dipyridamol echocardiography is a better predictor of subsequent cardiovascular death than SPECT. 


\section{Abnormalities of left ventricular wall motion in ischaemic heart disease: a comparison of Doppler echocardiography and magnetic resonance velocity mapping}

S P Karwatowski, S J Brecker, G Z Yang,

$M$ St John Sutton, $S$ R Underwood

Royal Brompton National Heart and Lung Hospital, London

Abnormal ventricular relaxation is an early marker of ischaemic heart disease. Diastolic flow through the mitral valve is a global measure of ventricular filling affected by ventricular relaxation and this is commonly assessed by Doppler ultrasonography. Regional abnormalities may be even more sensitive and we have compared regional measurements of myocardial velocity measured by magnetic resonance velocity mapping with mitral flow measured by Doppler ultrasonography. Eighteen patients (mean age 62 , range $42-78,16$ men) with coronary artery disease were studied after discontinuing cardiac medication. Twelve had suffered previous myocardial infarction. Doppler indices measured were peak mitral flow velocity during early diastole ( $\mathrm{E}$ vel) and atriosystolic contraction (A vel), isovolumic relaxation time (IVRT), and time to $E$ vel. The velocity ratio (E/A ratio) and peak rapid acceleration were derived. Cine magnetic resonance mapping of myocardial velocity perpendicular to a basal short axis plane was performed on the same day as the Doppler study and at similar heart rates. Velocity time curves were constructed in each of 16 sectors with measurement of the peak and mean early diastolic velocity and the number of segments with less than $50 \%$ of peak velocity $(\mathrm{N})$. These parameters thus measured the severity and extent of abnormality respectively. There was a strong inverse correlation between $N$ and $E$ vel $(r=-0.72, p<0.001)$ and between $\mathrm{N}$ and $\mathrm{E} / \mathrm{A}(\mathrm{r}=-0.68, \mathrm{p}<0.01)$, and a strong positive correlation between mean peak myocardial velocity and $\mathrm{E}$ vel $(r=0.8, p<0.001) ; A$ vel and IVRT did not correlate well.

Thus we have defined a new measure of regional diastolic ventricular function and have shown that regional abnormalities of wall motion during early diastole have a significant effect on left ventricular filling.

\section{Objective evaluation of skeletal muscle counterpulsation in a mock circulation model}

S S Shah, D V E Cumming, K Nishimura, C T Bowles, C W Pattison, J R Pepper, M H Yacoub

Royal Brompton National Heart and Lung Hospital, London

It is important to assess the haemodynamic benefit of extra-aortic skeletal muscle counterpulsation before its clinical use. This has been investigated in 12 animals (sheep) using a mock circulation model. The model consists of a closed system of two Windkessel compliance chambers joined together by a latex tube, around which the muscle can be wrapped in a manner similar to that in vivo. In each animal, the left latissimus dorsi muscle was electrically stimulated at a standard stimulation regimen: pulse amplitude three volts, burst frequency $35 \mathrm{~Hz}$, burst duration $240 \mathrm{~ms}$, pulse width $240 \mu \mathrm{s}$, in the 1:4 mode. The time course of stimulation extended to five months: two weeks (two animals); one month (three animals); three months (two animals); four months (two animals); five months (two animals). The length of the wrap was eight $\mathrm{cm}$ in all animals. The results show that counterpulsation at $1: 4$ and 1:2 modes is more effective than in the $1: 1$ mode. After one month of conditioning, the mean volume displacement $\left(\mathrm{cm}^{3}\right)$ was $18.4(0.4)$ in the $1: 4$ mode, $17.9(0.4)$ in the $1: 2$ mode, and $15.0(0.5)$ in the $1: 1$ mode, and the average peak pressure augmentation $(\mathrm{mm} \mathrm{Hg}$ ) was 17.5 $(0.9), 17 \cdot 1(0.5)$, and $14.4(0.5)$ respectively. A similar trend was found at the other time courses. Stimulation resulted in muscle fibre type transformation; at one month there was conversion to a $75 \%$ mixed fibre type population expressing fast and slow isoforms. Fibre type transformation was accompanied by loss of power from 14.8 Watts $/ \mathrm{kg}$ (unconditioned muscle) to 8.9 Watts $/ \mathrm{kg}$ (after five months of conditioning). The development of fatigue resistance was confirmed by the fact that in all animals the conditioned muscle completed a vigorous 30 minute fatigue test ending with a stimulation regimen of $5 \mathrm{~V}, 85 \mathrm{~Hz}$ in the $1: 1$ mode.

The results suggest that extra-aortic skeletal muscle counterpulsation does have the capacity to provide effective haemodynamic benefit and this may have a role in the treatment of patients with end stage cardiac failure.

\section{Long-term follow up of patients requring intensive in-hospital support who underwent urgent cardiac transplantation \\ D Mulcahy, M Fitzgerald, C Wright, M H Yacoub, K Fox \\ Royal Brompton National Heart and Lung Hospital, London; and Harefield Hospital, Middlesex}

There is concern about referring severely ill patients, who require active in hospital support, for cardiac transplantation, especially with the shortage of donor organs. Twenty six of 33 patients requiring such support with intravenous infusions of cardiac drugs and diuretics (32), in addition to balloon counterpulsation (16), peritoneal dialysis (7), pacing (3), and ventilation (2) as required, underwent urgent cardiac transplantation between January 85 and December 86 . Twenty (78\%) patients survived to hospital discharge, five more died at 10 weeks and 33, 36, 48 and 72 months. Fifteen patients remain alive and well at a mean of 63.5 months (range 56 to 78 months) after urgent cardiac transplantation $(57.7 \%$ of those undergoing cardiac transplantation). Ten are working full time. They had a mean pretransplant ejection fraction of $11 \%$, and a mean of 2.7 infusions (range 1 to 5) of inotropic/vasodilator drugs. Three required balloon counterpulsation and four emergency resuscitation at some point during admission before transplantation. Sixty six per cent had derangement in liver function, with $>50 \%$ having renal dysfunction.

The long term results of severely ill patients undergoing urgent cardiac transplantation are very good, both in terms of survival and quality of life. Patients not requiring renal dialysis or balloon counterpulsation before transplantation seem to do particularly well. There seems little reason to exclude those who require inotropic and other supports or those who have evidence of non-critical hepatic or renal dysfunction from cardiac transplantation. 
Predictive value of pulmonary haemodynamic measurements in patients accepted for cardiac transplantation

\author{
J C Doig, K Nimkhedkar, C J Hilton, J H Dark, \\ R S Bexton \\ University Department of Cardiology, Freeman \\ Hospital, Newcastle upon Tyne
}

This study investigated the predictive value of pulmonary haemodynamic measurements on survival after, or while awaiting, cardiac transplantation. In patients with raised resistances, the acute response to pharmacological manipulation was assessed to determine whether successful lowering of resistances conferred an improved survival potential. Right heart catheterisation was performed in $\mathbf{7 8}$ patients accepted on to our transplant programme. Transplant survivors $(S, n=58$ ) were compared with patients dying within 30 days of operation $(\mathrm{TxD}, \mathrm{n}=8)$ and on the waiting list (WLD, $\mathbf{n}=12$ ). Levels of pulmonary hypertension (right ventricular systolic: $\mathrm{S} 49$ (14), TxD 54 (12), WLD 58 (16) $\mathrm{mm} \mathrm{Hg}$, mean pulmonary artery: S34 (10), TxD 35 (9), WLD 40 (12) mm Hg) were not significantly different, nor predictive of outcome. Transpulmonary gradient (TPG) was higher in patients who died (S9 (4), TxD 11 (3), WLD 13 (7) $\mathrm{mm} \mathrm{Hg}, \mathrm{p}=0.015$ ) and TPG $\geqslant 12$ was predictive of mortality, $\mathrm{p}=0.016$, with sensitivity (sens) $58 \%$, specificity (spec) $76 \%$, predictive accuracy (pa $71 \%$ ). Pulmonary arteriolar resistances (PAR) were lower in survivors (S 2.4 (1.3), TxD 3.2 (2.9), WLD 3.6 (2.3) Units (U), $\mathrm{p}=0.008)$ and PAR $\geqslant 4$ was predictive of mortality, $\mathrm{p}=0.006$; sens $42 \%$, spec $91 \%$, pa $78 \%$. Indices of PAR were lower in survivors (S $4 \cdot 2(2 \cdot 1)$, TxD 5.4 (3.2), WLD $6.5(3.9) \mathrm{U} \times$ sq.m, $\mathrm{p}=0.005)$ and PAR index $>8$ predicted mortality, $\mathrm{p}=0.014$; sens $32 \%$, spec $93 \%$, pa $77 \%$. All groups had similar cardiac indices, mean right atrial, and pulmonary artery wedge pressure measurements. Thirteen patients had raised PAR $>4 \mathrm{U}$. Seven underwent acute pharmacological manipulation, and PAR fell below 4 $\mathrm{U}$ in four patients, two of whom died. In total, eight patients with PAR $>4$ were transplanted and three died $(38 \%)$.

This study shows the importance of pulmonary haemodynamic indices in the assessment of potential cardiac transplant recipients. Patients with TPG $\geqslant 12 \mathrm{~mm} \mathrm{Hg}$, $P A R \geqslant 4 \mathrm{U}$, or PAR index $\geqslant 8 \mathrm{U} \times \mathrm{sqm}$ had reduced survival potential irrespective of whether transplantation was performed. In patients with raised PAR, the initial value is of greater predictive value than the response to pharmacological manipulation.

\section{Surgical management of end stage heart disease: is conventional surgery still an option?}

F Ciulli, T A H English, N Caine, P Mullins, J Parameshwar, P M Schofield, J Wallwork, S R Large Transplant Unit, Papworth Hospital, Cambridge

In a selected group of patients referred for cardiac transplantation, conventional cardiac surgery may still represent an important alternative method of treatment. We audited our experience of this approach between January 1979 and June 1991 . Of the 1398 referrals for cardiac transplantation, 52 patients $(3.7 \%)$ were considered suitable for conventional cardiac surgical intervention, while 433 underwent cardiac transplantation. Of the 52 patients treated with conventional cardiac surgery, $40(76.9 \%)$ underwent coronary artery bypass grafting, including 15 left ventricular aneurysmectomies, $10(19.2 \%)$ cardiac valve replacements, and two $(3.8 \%)$ had valve replacement and coronary artery bypass graft procedures. In hospital mortality ( $<30$ days) occurred in seven $(13.5 \%$ ) patients in the valve replacement group and two $(3.8 \%)$ in the coronary artery bypass group. The overall actuarial survival for the patients undergoing heart transplantation at one, three, and five years respectively was $75 \%(2 \cdot 1), 69 \%(2 \cdot 4)$ and $62 \%(2 \cdot 8)$. The overall actuarial survival for the patients undergoing conventional surgery at one, three, and five years was $80 \cdot 2 \%(5 \cdot 6), 69 \cdot 2 \%(6 \cdot 7)$, and $55 \cdot 8 \%(7 \cdot 6)$. Of the 45 hospital survivors after conventional surgery, 12 patients $(26.7 \%)$ eventually received transplantation at a mean of 28.8 months $(25.4)$. The one, three, and five year actuarial survival for the $\mathbf{4 0}$ patients who had coronary artery bypass surgery is $89.6 \%(4.9), 80.8 \%(6.6)$, and $63.0 \%(8 \cdot 7)$. Three patients of the $10(33.3 \%)$ in the valve replacement group died within 30 days of surgery and four died between five and 19 months after surgery and one patient underwent transplantation at 24 months; the remaining two are well at 119 and 61 months. New York Heart Association (NYHA) classification improved in most hospital survivors after conventional surgery (NYHA class preoperatively, III $=20$ patients $(38 \%), I V=32(62 \%)$ and postoperatively, I $=12$ patients $(43 \%), \quad$ II $=14$ $(50 \%)$, III $=$ two patients $(1 \%))$.

In a selected group of patients referred for transplantation, conventional surgery may represent a suitable option offering improved survival and functional state.

Accelerated coronary sclerosis in children after cardiac transplantation: what role do oral steroids have?

R Radley-Smith, M H Yacoub

National Heart and Lung Institute, London; and

Harefield Hospital, Harefield, Middlesex

Since August 1984, 98 children under the age of 15 years have undergone cardiac transplantation at Harefield Hospital. Their ages were between seven days and 14.8 years (mean 6.4 years). The indications were cardiomyopathy in 71 , congenital heart disease in 25 and Kawasaki's disease in two. After transplantation, patients were maintained on cyclosporin A and azathioprine only. Routine steriods were not given. Acute rejection in this group was detected by non-invasive methods and routine biopsies were not performed. Sixty one patients have been followed up for more than one year ( 1.2 to 7.4 years) and $58(95 \%)$ have undergone routine cardiac catheterisation and selective coronary angiography, irrespective of age, on 137 occasions. Right heart pressures and left ventricular end diastolic pressure was normal in all patients. In an attempt to diagnose accelerated coronary sclerosis, the coronary angiograms were reviewed by at least two independent observers. Only 1/137 angiogram was judged to be abnormal. This occurred six years after transplantation. One child, who did not undergo his routine angiogram at 12 months, died at 18 months from coronary occlusion and another child who died of chicken pox, six months after a "normal" angiogram was found to have coronary sclerosis at post mortem. Both these children were receiving steroids for conditions unrelated to their transplant. None of the other four late deaths were found to have coronary sclerosis at post mortem. Risk factors for coronary sclerosis were reviewed. There was no correlation with the number of rejection episodes but $2 / 4$ children receiving routine steroids, as opposed to $1 / 57$ not on steroids, developed this life threatening complication.

In the absence of routine steroids, accelerated coronary sclerosis is rare in children after cardiac transplantation. 
Creatine kinase (CK-MB) isoforms as a non-invasive marker of significant acute allograft rejection after heart transplantation

A Caforio, $\mathrm{S} H$ Jennison, $M$ Hossein-Nia, $\mathrm{P}$ Brown, C M Corbishley, A J Murday, D W Holt, W J McKenna Cardiac Transplant Programme, St George's Hospital, London

Frequent routine surveillance by right ventricular endomyocardial biopsies is necessary in cardiac transplant patients in their first postoperative year, to detect significant acute cardiac allograft rejection. Biopsy procedures carry an associated morbidity, a tendency to sample old biopsy sites, and inconvenience to the patient. Detection of the creatine kinase (CK)-MB2 isoform in plasma, as a sensitive marker of myocardial cell injury, is now possible. Measurement of the $\mathrm{MB} 2 / \mathrm{MB} 1$ ratio has been used in the early diagnosis of acute myocardial infarction, when the total $\mathrm{CK}-\mathrm{MB}$ isoenzyme activity is within the normal range. This study assessed CK-MB isoform measurement as an early non-invasive marker of allograft rejection after cardiac transplantation. Plasma samples were collected from 16 orthotopic heart transplant recipients before endomyocardial biopsy. The CK-MB isoforms were determined by high resolution agarose gel electrophoresis and quantified by densitometric scanning. 9/16 patients had 10 episodes of allograft rejection; the total $\mathrm{CK}-\mathrm{MB}$ isoenzyme activity during this time was not raised (median $3.6 \mathrm{U} / 1$ (range 2.2 to 10.6 )) but the $\mathrm{MB} 2 / \mathrm{MB} 1$ ratios increased (median 2.4 , range 1.0 to 6.6 ), compared with no rejection (median 1.2 , range 0.3 to 2.3 , p $<0.003$, Mann-Whitney $U$ test). Moreover, the $M B 2 / M B 1$ ratio had increased before histological changes seen on biopsy in 6/9 patients (mean 10 days), indicating that either myocardial cell injury was present but had been missed by biopsy, or was developing before histological changes were apparent.

Total CK-MB isoenzyme activity is not raised during allograft rejection, but the $M B 2 / M B 1$ ratio is significantly increased, suggesting that its measurement as a predictive non-invasive marker of allograft rejection warrants further evaluation.

Effects of physical training on skeletal muscle metabolism in chronic heart failure: experimental and human study

\section{$S$ Adamopoulos}

Department of Cardiovascular Medicine, John Radcliffe Hospital and MRC Biochemical and Clinical Magnetic Resonance Unit, University of Oxford

Muscle deconditioning has been considered one of the possible mechanisms for the intrinsic skeletal muscle metabolic changes in chronic heart failure (CHF). To investigate the effects of physical training on the skeletal muscle metabolism we studied 12 patients (human study, HS) with ischaemic stable $\mathrm{CHF}$ and 32 female Wistar rats (experimental study, ES) after coronary artery ligation $(n=22)$ or sham operation $(n=10)$. Six weeks after the operation infarcted rats were allocated randomly to either another 6 weeks of training on a treadmill $(T, n=11)$ or non-training (NT, $\mathrm{n}=11$ ). Muscle $\mathrm{pH}$, inorganic phosphate $(\mathrm{Pi})$, phosphocreatine $(\mathrm{PCr})$ utilisation, and ADP levels were evaluated before and after 8 weeks of home based bicycle exercise training in patients in a crossover randomised order and 12 weeks after the operation in both infarcted and sham operated rats. Serial ${ }^{31} \mathrm{P}$ spectra of the calf muscle were obtained at rest, throughout plantar flexion at incremental workloads until exhaustion and during the recovery period (HS) and during sciatic nerve stimulation $(1 \mathrm{~Hz})$ at both supramaximal and $200 \mathrm{~g}$ tension (ES). Rats were divided into congestive (C) and noncongestive (NC) groups according to lung/body weight ratio and enzymatic assays were performed in the contralateral leg to measure glutamate pyruvate transferase (GPT) and the mitochondrial oxidative enzymes citrate synthase (CS) and $\beta$-hydroxyacyl CoA dehydrogenase (BOAC).

Training produced an increase $(p<0.002)$ in incremental plantar flexion exercise time. PCr utilisation and ADP levels during exercise were reduced significantly ( $p<0.003$, ANOVA for repeated measures) by training at all matched submaximal workloads and at peak exercise. The PCr and ADP recovery half times were significantly faster after training $(p<0.05)$. Compared with all other groups the NT C rats produced more acid at $200 \mathrm{~g}$ $(p<0.05$, ANOVA) and at maximal tension $(p<0.01)$ and had lower PCr at $200 \mathrm{~g}(\mathrm{p}<0.01)$. The NT C group had significantly lower CS in skeletal muscle than the T C or $\mathrm{T}$ NC and sham groups ( $p<0.001)$. There was a significant increase in GPT in the training group compared with the non-training group $(p<0.03)$. There were no differences in BOAC.

I conclude that (a) skeletal muscle metabolic abnormalities in CHF depend on both congestive failure and physical deconditioning and (b) physical training can reverse the excessive $\mathrm{PCr}$ depletion and reduce ADP levels during exercise and can accelerate the rate of $\mathrm{PCr}$ resynthesis and ADP disappearance during recovery, indicating an improvement in the impaired oxidative metabolism of skeletal muscle seen in CHF.

Measurement of renal blood flow by continuous renal vein thermodilution: a new technique for the investigation of human cardiovascular reflexes

\section{G A Haywood}

Department of Cardiological Sciences, St George's Hospital Medical School, London

This study tested the hypothesis that cutting the cardiac ventricular afferent nerve fibres at heart transplantation results in abnormal cardiovascular reflex responses to exercise in heart transplant recipients. In animals the regulatory effects of the cardiac afferent fibres exert their primary influence on the renal circulation. A continuous thermodilution technique applied in the left renal vein was developed and validated. This allowed rapid measurements of changes in renal blood flow to be made in 30 subjects (10 heart transplant recipients, 10 patients with NYHA class II heart failure, and 10 control subjects) during exercise. Measurements by the thermodilution technique correlated well with absolute blood flow in an in vitro model for blood flows ranging from 55 to $885 \mathrm{ml} / \mathrm{min}$ $(r=0.99)$, with renal arterial blood flow measured by electromagnetic flow probe in a pig model $(r=0.92)$, and with simultaneous measurements of renal blood flow by corrected iodohippurate clearance in humans $(r=0.8)$. The percentage fall in renal blood flow at peak exercise was significantly greater in heart transplant recipients than in controls $(45(5) \% v 32(4) \% \mathrm{p}<0.05)$, as was the percentage increase in renal vascular resistance (transplants $116(19) \% v$ controls $78(17) \%, p<0.05)$. Renal haemo- 
dynamics during exercise in the heart failure group were not significantly different from those in controls.

Continuous renal vein thermodilution is a useful technique for the investigation of human cardiovascular reflexes. Ventricular cardiac denervation resulting from heart transplantation alters cardiovascular reflexes during exercise, resulting in increased vasoconstrictor drive to the kidneys. This mechanism may contribute to renal impairment after heart transplantation.

\section{Gap junctions in normal, ischaemic and hypertrophied human ventricular myocardium: a quantitative and descriptive study of gap junction intercellular communication pathways}

\section{N S Peters \\ National Heart and Lung Institute, and University College, London}

Antibodies raised to a portion of the cardiac gap-junctional protein were used in an immunohistochemical procedure in combination with laser scanning confocal microscopy to localise gap junctions with a clarity not previously possible through thick volumes of human myocardium. To explore the structural basis for electromechanical dysfunction in myocardial ischaemia, infarction, and hypertrophy this technique was applied to study the organisation of gap junctions in ventricular myocardial samples obtained during surgery. Gap junctions in normal human working left ventricular myocardium are confined to the intercalated discs, with a gap-junctional surface density of 0.00508 $\mu \mathrm{m}^{2} / \mu \mathrm{m}^{3}$ myocardial volume. The right ventricle has similar characteristics. The border zones of healed myocardial infarcts have a considerably disturbed gap junction distribution. There is a reduced gap junction surface density in ischaemic ventricular myocardium $(p=0.02)$ and in hypertrophy $(p=0.05)$. When the larger cell volume of these pathological tissues is taken into account gapjunctional content per cell shows a significantly reduced value only when ischaemic ventricle is compared with normal ventricle $(p=0.02)$, suggesting that the reduced surface density is a feature of ischaemia, and not simply hypertrophy, in the ischaemic heart.

These disturbances of the gap-junctional organisation may account for abnormalities of impulse propagation in ischaemic and hypertrophied ventricular myocardium and may contribute to arrhythmic tendency and mechanical inefficiency.

Clinical applications of molecular genetic analysis in familial hypertrophic cardiomyopathy

\section{Hugh Watkins}

Cardiology Division, Brigham and Women's Hospital, and Harvard Medical School, Boston, Massachussetts, USA, and Department of Cardiological Sciences, St George's Hospital Medical School, London

Familial hypertrophic cardiomyopathy (FHC) in two families has been shown to be caused by cardiac myosin heavy chain (MHC) gene missense mutations. However, the disease is genetically and clinically heterogeneous and neither the fraction of FHC caused by myosin mutations nor accurate indicators of disease prognosis have been identified. To address these issues a technique incorporating the poly- merase chain reaction and RNase protection assays was developed for screening the $\beta$ MHC gene for mutations. Twenty four probands from unrelated families with FHC were studied. Seven $\beta$ cardiac MHC gene mutations that cause FHC in 11 families were identified. All were missense mutations clustered in the head and head/rod junction of the molecule; six mutations involved a change in charge of the altered residue. In affected individuals specific mutations were associated with significantly different life expectancy. In the individuals with the two most severe mutations the mean age at death was 33 years. The one mutation that did not affect the charge was associated with near normal survival.

This new technique makes a genetic diagnosis possible in approximately $50 \%$ of families with FHC, regardless of family size. Definition of the disease-causing mutation allows preclinical diagnosis and provides important prognostic information.

Similar prevalence of enterovirus genome within the myocardium of patients with idiopathic dilated cardiomyopathy and controls shown by the polymerase chain reaction

P J Keeling, S Jeffrey, A L P Caforio, R Taylor, M J Davies, G F Bottazzo, W J McKenna

St George's Hospital Medical School, London

Enteroviral infection remains an important hypothesis for the pathogenesis of idiopathic dilated cardiomyopathy. Recent studies have suggested that with highly sensitive and enteroviral-specific techniques enteroviral genome can be detected within the myocardium of 10 to $20 \%$ patients with idiopathic dilated cardiomyopathy: however, these studies have failed to provide adequate control data. In this study we used an enteroviral-specific gene amplification polymerase chain reaction technique to detect enteroviral genome within the myocardium taken from 50 patients with dilated cardiomyopathy, 41 with other forms of heart disease, and 34 from coroners' necropsy cases. Enteroviral genome was detected in $6 / 50(12 \%)$ patients with dilated cardiomyopathy and 13/75 (17\%) of the controls (NS). No differences were found in dilated cardiomyopathy patients with or without myocardial enteroviral genome in respect of age, duration of symptoms, proportion of patients with a premorbid acute viral illness, excess alcohol consumption, New York Heart Association functional class, measures of left ventricular function, or endomyocardial histology. Within the control group enteroviral genome was detected in $3 / 15(20 \%)$ patients with ischaemic heart disease, $2 / 19(10.5 \%)$ with valvar heart disease, $1 / 5(20 \%)$ with specific heart muscle disease, $0 / 2(0 \%)$ with congenital heart disease, and $7 / 34(20.6 \%)$ cases of sudden death. During 2 to 52 month follow up (mean 22) 15/44 $(34 \%)$ patients without myocardial enteroviral genome and $2 / 6(33 \%)$ with myocardial enteroviral genome died suddently or required orthotopic heart transplantation for progressive heart failure.

Enteroviral genome was detected within the myocardium in a similar proportion of patients with idiopathic dilated cardiomyopathy and controls. This finding does not support the hypothesis that persistent enteroviral infection is of pathogenic or prognostic importance in dilated cardiomyopathy but is most consistent with enterovirus being a common environmental pathogen. 
Mis-sense mutations in myosin suggest a pathogenetic mechanism in familial hypertrophic cardiomyopathy

\section{H Watkins, A Rosenzweig, J G Seidman, C E Seidman, W J McKenna}

St George's Hospital Medical School, London; and Brigham and Women's Hospital, Boston, USA

Cardiac myosin heavy chain (MHC) gene mutations have been implicated as the cause of familial hypertrophic cardiomyopathy (FHC), but neither the types of cardiac MHC mutations that can cause FHC, nor the mechanism by which these mutations cause disease, has been defined. Previously identified cardiac MHC gene mutations suggest very different mechanisms by which altered MHC polypeptides produce this disease. Affected persons in two families have a mis-sense mutation in a highly conserved residue of the $\beta$ cardiac MHC gene while affected individuals in another family have an $\alpha / \beta$ cardiac MHC hybrid gene. We have now systematically screened the $\beta$ cardiac MHC genes of 24 unrelated individuals with FHC and identified seven $\beta$ cardiac MHC gene mutations that cause FHC in 11 of the families. All seven of these mutations are mis-sense mutations in the head or head and rod junction regions of $\beta$ cardiac MHC. Moreover, affected persons with the previously identified $\alpha / \beta$ hybrid MHC gene were found to have a coexistent mis-sense mutation that is itself responsible for FHC. Six of the seven mutations involve a charge change in the encoded amino acid and so are particularly likely to cause regional conformational change in the polypeptide. The identification of multiple new FHC-causing mis-sense mutations clustered within the MHC polypeptide suggests that mutationally altered $\mathrm{MHC}$ peptides act as "poison polypeptides." Analogous mis-sense mutations in a nematode MHC gene are known to produce a dominant phenotype of paralysis by incorporation of mutant heavy chains into assembling thick filaments which then disrupt sarcomere assembly. The presence of multiple different mis-sense mutations in the $\beta$ MHC gene suggests a relatively high mutational rate, such that most FHC may be attributable to relatively recent mutations, rather than to a founder effect. This is in keeping with the significant premature mortality in FHC, which will tend to remove disease-causing mutations from the population, whereas new mutations account for the persistence of FHC.

A high incidence of de novo myosin mutation may account for some of the apparently sporadic hypertrophic cardiomyopathy found in people with no known affected relatives.

\section{Myocardial nitric oxide production could contribute to cardiac depression in endotoxaemia and allograft rejection}

\section{A M Shah, H G Evans, M J Lewis}

Cardiovascular Research Group (Departments of Cardiology and Pharmacology and Therapeutics), University of Wales College of Medicine, Cardiff

Endothelium derived relaxing factor (EDRF or nitric oxide (NO), produced by constitutive NO synthase) released from endocardial endothelium in isolated cardiac preparations raises myocardial cyclic guanosine monophosphate concentrations (GMP) and shorten contractile twitch duration without major effect on early systolic parameters, for example unloaded shortening velocity $\left(\mathrm{V}_{\max }\right)$. A different, calcium independent NO synthase enzyme inducible by cytokines, for example interleukin- $1 \beta$ (IL-1), has recently been isolated in mammalian myocytes.
The effects of IL- 1 on contraction and cyclic GMP content in isolated ferret papillary muscles $(\mathrm{n}=33$; with $1 \mu \mathrm{M}$ acebutolol and $10 \mu \mathrm{M}$ indomethacin) were therefore studied. After three hours incubation, IL-1 $(10 \mathrm{ng} / \mathrm{ml})$ reduced time to peak isometric tension (tPT) by $14.0(3.0) \%$ (mean (SE) and peak tension (PT) by $6.9(1.6) \%$, and increased cyclic GMP by $95.8 \%(p<0.05 ; n=6)$, without altering $V_{\max }$. These effects were independent of the endocardium. The contractile effects of IL-1 were inhibited (tPT -3.5 $(2.0) \%$ and $-3.7(1.4) \%$ respectively (NS; $n>6)$ ) in the presence of $\mathrm{L}-\mathrm{N}^{\mathrm{G}}$-monomethylarginine (L-NMMA $(50 \mu \mathrm{M})$ for $30 \mathrm{~min})$ or dexamethasone $((3 \mu \mathrm{M})$ for 3 hours), which both inhibit inducible NO synthase. The IL-1 induced increase in cyclic GMP was $10.4 \%(n=6)$ in the presence of L-NMMA and $52.1 \%(n=6, p<0.05)$ in the presence of dexamethasone.

Thus IL-1, probably by inducing myocardial NO synthase, mimics the characteristic EDRF effect of shortening the duration of myocardial contraction, which could reduce stroke volume and contribute to cardiac depression in immune mediated shock, for example, endotoxaemia and allograft rejection.

\section{Beneficial effects of growth hormone replacement therapy on myocardial function}

\author{
M Shahi, S Besh yah, P Sharp, D Johnston, R A Foale \\ St Mary's Hospital, London
}

Patients with hypopituitarism who have replacement therapy with thyroxine and adrenal steroids but not growth hormone have increased cardiovascular mortality. A recent report has found that a substantial number of these patients have impaired myocardial function, in particular a reduced exercise time, electrocardiographic abnormalities on exercise, and abnormal left ventricular diastolic function. To assess whether growth hormone replacement therapy $(0.06 \mathrm{IU} / \mathrm{kg}$ of body weight given subcutaneously once daily) improves these abnormalities, we conducted a double blind, placebo controlled study on 26 adults with hypopituitarism for six months ( 12 men, 14 women; mean age 45 (12) years). Cardiac structure and function were assessed with cross sectional echocardiography and Doppler ultrasound, and treadmill exercise tolerance was assessed with the Bruce protocol. During the six month study period there were no changes in left ventricular mass index in either the placebo group $\left(88(18) \mathrm{g} / \mathrm{m}^{2}\right.$ at baseline, $92(14) \mathrm{g} / \mathrm{m}^{2}$ at six months) or the growth hormone treated group $\left(96(21) \mathrm{g} / \mathrm{m}^{2}\right.$ and $\left.94(22) \mathrm{g} / \mathrm{m}^{2}\right)$. Of the indices of left ventricular diastolic function, isovolumic relaxation time improved in the growth hormone treated group (102 (15) ms at baseline, 92 (11) ms at six months, $\mathrm{p}<0.05)$ but not in the placebo group (99 (17) ms and 93 (13) ms, $p>0.03)$. No difference was found in the indices of left ventricular filling (E:A ratio) in either group. Exercise duration increased in the growth hormone treated group $(8.4(1.9) \mathrm{min}$ at baseline, $9.4(1.5) \mathrm{min}$ at six months, $\mathrm{p}<0.04)$ but not in the placebo group $(8.7(4.4) \mathrm{min}$ and $8.7(4 \cdot 1) \mathrm{min}, \mathrm{p}>0.9)$. No change was found in the ratepressure product in either group after six months. Four patients in the growth hormone treated group and three in the placebo group developed planar ST depression $>0.1 \mathrm{mV}$ during exercise testing at baseline which did not change in either group after six months.

These findings suggest that growth hormone replacement therapy even over a short period of time has several potentially beneficial cardiac effects in patients with hypopitituitarism and may possibly in the long-term reduce their increased cardiovascular mortality. 
Effects of type 1 diabetes on left ventricular size and function: Doppler echocardiographic study of identical twins

\author{
S S S Lo, R D G Leslie, M G St John Sutton \\ Diabetic Unit, Westminster Hospital; and Royal \\ Brompton Heart and Lung Hospital, London
}

Heart failure occurs more frequently in diabetic than nondiabetic patients despite a similar extent of coronary artery disease, suggesting the presence of a specific diabetic heart disease. In order to elucidate the nature of this heart disease previously described in heterogeneous populations of diabetics, we assessed left ventricular size and function by 2D-Doppler echocardiography in 36 young (mean 26, range 10 to 37 years) normotensive identical twin pairs discordant for type 1 diabetes (mean duration 14 years). End diastolic volume (EDV), end systolic volume (ESV) and left ventricular mass were calculated from echo images and indexed. Diastolic function was measured as: the ratio of transmitral blood flow velocities during early and late left ventricular filling (E/A), time-velocity integrals of early ( $E$ integral) and late (A integral) left ventricular filling, and percentage of diastolic filling contributed by atrial systole (A\%). Diabetic twins, compared with nondiabetic twins, had lower EDV and ESV (72 (20) $v 76$ (19) $\mathrm{ml} / \mathrm{m}^{2}, \mathrm{p}<0.001$ and $\left.31(8) v 36(14) \mathrm{ml} / \mathrm{m}^{2}, \mathrm{p}<0.02\right)$ and lower $\mathrm{E} / \mathrm{A}$ ratio $(1.4(0.3) v 1.6(0.3), \mathrm{p}<0.0005)$. Although the total time-velocity integrals of diastolic filling were similar between the twins, the diabetic twins had higher A integral (55 (18) v 47 (10), p < 0.005) and higher A\% (32 (5) $v 29(5) \%, p<0.005)$. Left ventricular mass, stroke volume, and relative wall thickness were similar between diabetic and non-diabetic twins. The smaller left ventricular size and altered left ventricular diastolic function in the diabetic twins were not causally related to disease duration, microvascular complications, or glycaemic control.

Young normotensive type 1 diabetics have decreased left ventricular chamber volume and subclinical diastolic dysfunction but retain normal systolic function, consistent with the existence of a specific diabetic heart disease.

\section{The duration and nature of isovolumic relaxation determine the Doppler $A / E$ ratio in left ventricular disease}

\section{S J D Brecker, C H Lee, D G Gibson Cardiac Department, Royal Brompton National Heart and Lung Hospital, London}

It is widely recognised that loading conditions, age, and heart rate influence peak mitral flow velocity and the Doppler A/E ratio. To assess possible additional effects of abnormal relaxation, we examined left ventricular (LV) filling with Doppler and $M$ mode echocardiography in 47 patients with ischaemic heart disease (IHD), 35 with LV hypertrophy ( $\mathrm{LVH}$ ), and in 26 normal people. We digitised $M$ modes and measured incoordinate relaxation as \% change in $\mathrm{LV}$ dimension before mitral valve opening, isovolumic relaxation time (IVRT), and rates of $\mathrm{LV}$ dimension increase and posterior wall thinning. Left ventricular end diastolic pressure was measured at cardiac catheterisation. The $\mathrm{A} / \mathrm{E}$ correlated with age in normal subjects $(r=0.74)$, to a lesser extent in LVH $(r=0.41)$, but not significantly in IHD. In LVH and those patients with ischaemic heart disease without $\mathrm{LV}$ dilatation, A/E was correlated both with IVRT $(r=0.67$ and 0.68 respectively), and with incoordinate relaxation $(r=0.64$ and 0.58 ). In those ischaemic heart disease patients with LV dilatation (end diastolic dimension $>6 \mathrm{~cm}$ ), the influence of incoordination was lost and IVRT became the dominant influence upon $\mathrm{A} / \mathrm{E}(\mathrm{r}=0.83)$ (all $\mathrm{p}$ values $<0.01)$. This was despite considerable incoordinate relaxation being evident in this subgroup. Stepwise regression confirmed that weak correlations of $\mathrm{LV}$ end diastolic pressure and $R R$ interval with $A / E$ became insignificant once IVRT had been taken into account. In 16 patients who had two examinations on different occasions, changes in IVRT were matched by changes in $A / E$, predicted by the relation derived from the group as a whole.

The influence of filling pressure and $R R$ interval on $A / E$ in patients with $\mathrm{LV}$ disease acts through an effect on IVRT. Age is an important influence in normal people, but this effect is attenuated in LVH and lost in IHD. Thus the filling pattern is effectively determined before mitral valve opening from two discrete properties of IVRT: its duration, and degree of incoordination during it, when cavity size is normal.

\section{Breathlessness in microvascular angina}

\section{A C Tweddel, R Carter, I Hutton}

Royal Infirmary, Glasgow

In patients with microvascular angina, there is some evidence from studies of plethysmography, of widespread microvascular abnormalities. As well as exertional chest pain, all of these patients complain of breathlessness, with no evidence of airways obstruction or resting left ventricular dysfunction. Progressive exercise testing was performed in 11 age and sex matched controls and 11 patients (two men), in whom the diagnosis of microvascular angina was established on the basis of exertional chest pain, abnormal thallium scans, and an attenuated myocardial flow response to a vasodilator challenge, with angiographically entirely normal epicardial vessels. Symptom limited exercise was performed with on line ventilation and expired gas analysis, measuring minute by minute ventilation, oxygen consumption and carbon dioxide production, and arterial blood gas values, using a transcutaneous system. Anaerobic threshold was calculated by curve fitting a plot of oxygen consumption against carbon dioxide production. Compared with controls $49.73 \mathrm{SD}(7.3)$ in patients with microvascular angina, anaerobic threshold was $41.55(5.82), p<0.016$ ( $\%$ predicted $\mathrm{Vo}_{\mathrm{cmax}}$ ) but still within normal limits. Maximal oxygen consumptions, as percentage of predicted, was reduced $59.73(16.51)$ compared with 86.55 $(4.91) \%, p<0.003$ and the mean ventilatory response $\mathrm{VE} / \mathrm{VCO}_{2}$ was significantly increased $35.20(8.01) v 26.49$ (3.08) $\left(1 / 1 \mathrm{CO}_{2}\right.$ output), $\mathrm{p}<0.016$.

These results suggest that in patients with microvascular angina on maximal exercise there is significant "wasted ventilation," consistent with areas of the lung being underperfused and accounting for the sensation of breathlessness. 


\section{Coronary arterial endothelium dependent vasodilator responses in syndrome $X$}

D R Holdright, D Clarke, D C Lindsay, P A Poole-Wilson, $\mathrm{K}$ M Fox, P Collins

National Heart and Lung Institute, Royal Brompton

National Heart and Lung Hospital, London

Certain features in patients with syndrome $\mathrm{X}$ (SX)namely, chest pain, positive exercise test, and angiographically normal coronary arteries-are suggestive of transient myocardial ischaemia. A functional abnormality at the level of the resistance vessels, possibly due to impaired endothelium dependent vasodilatation, may explain the syndrome. With an intracoronary Doppler probe to measure coronary blood velocity and quantitative angiography to determine coronary artery diameter, endothelium dependent responses to acetylcholine (ACh) and substance $P(S P)$ were assessed in 10 SX patients and nine controls (atypical chest pain, negative exercise test, and angiographically normal epicardial coronary arteries). Intracoronary infusions of $\mathrm{ACh}\left(10^{-7} \mathrm{M}\right.$ and $\left.10^{-6} \mathrm{M}\right)$ and $\operatorname{SP}(5,10$, and $25 \mathrm{pmol} / \mathrm{min})$ were given. Flow (area $\times$ velocity) was determined before infusion and at peak response to produce a flow ratio (peak:baseline flow). Epicardial area was not significantly altered by $\mathrm{ACh}$, but was increased with SP by (mean (SEM)) $51 \cdot 2(17 \cdot 3) \%$ (in the SX patients, $p<0.05)$, and $53.1 \quad(7.3) \%$ (in the controls, $\mathrm{p}<0.05)$. Flow ratios were greater with $\mathrm{ACh}$ $(2.71(0.32$ in the SX patients, $p<0.01)$ and $2.88(0.42)$ (in the controls, $\mathrm{p}<0.05)$ ) than with SP $(1.88(0.13)$ (in the SX patients, $\mathrm{p}<0.05$ ) and $2.42(0.43)$ (in the controls, $\mathrm{p}<0.05)$ ). There was no significant difference between SX patients and controls with either drug.

Endothelium dependent vasodilator responses are not impaired in SX patients compared with a control group, and this highlights the different effects of ACh and SP on the coronary bed. If a functional abnormality exists then other mechanisms must be considered.

\section{Effect of hyperventilation and mental stress on coronary blood flow in patients with syndrome $X$}

A Chauhan, P Mullins, S Thuraisingham, G Taylor, A Jawhari, M C Petch, P M Schofield

Cardiac Unit, Papworth Hospital, Cambridge

The mechanism of chest pain in patients who present with chest pain, a positive exercise test, and normal coronary arteries (syndrome $\mathbf{X}$ ) is not clear. Syndrome $\mathbf{X}$ is a heterogeneous disorder and the various proposed theories include abnormalities of coronary vasodilator reserve in some patients whereas psychological, hyperventilation, and oesophageal factors have been reported in others. We investigated the effect of hyperventilation and mental arithmetic stress in a group of 18 syndrome $X$ patients. All cardiac medications were stopped 48 hours before the tests. All patients first underwent hyperventilation and mental stress test on the ward 24 hours before the cardiac catheter. With a standard femoral approach a $3.6 \mathrm{~F}$ intracoronary Doppler catheter (Schneider, UK) was positioned in the proximal left anterior descending coronary artery. The resting coronary blood flow velocity was measured with the patient in the supine position and breathing normally. Hyperventilation followed by mental arithmetic stress test was performed in the first 10 patients and the order was reversed in the remaining eight. During the tests performed on the ward, 10 patients experienced their usual chest pain on hyperventilation and 10 patients during or soon after the mental stress test but none of these episodes produced appreciable ischaemic electrocardiographic changes. Seven of the 10 patients in both groups experienced chest pain with both hyperventilation and mental stress. This exact pattern was repeated during the cardiac catheter with hyperventilation and mental stress. Hyperventilation produced a significant increase in heart rate (77 (SD) (17) $v 85$ (16) $\mathrm{p}=0.001$ ) but no significant change in systolic blood pressure. The rate-pressure product increased significantly from 9267 (2216) to 10528 (3045) $(p=0.009)$. Coronary blood flow velocity, however, decreased significantly from $7.6(4.9) \mathrm{cm} / \mathrm{s}$ to $6.6(5) \mathrm{cm} / \mathrm{s}$ ( $\mathrm{p}=0.02$, paired $t$ test). On mental stress test there was no significant difference in heart rate $(78(16) v 80(16))$, systolic blood pressure (132 (24) $v 135(26)$ ), or coronary blood flow velocity $(8 \cdot 3(5 \cdot 2) v 7 \cdot 4(4 \cdot 5))$.

In syndrome $X$ patients although both hyperventilation and mental stress can produce chest pain, only hyperventilation appears to significantly reduce coronary blood flow.

\section{Effects of hormone deficiency on cardiovascular risk markers in women}

\section{J C Stevenson, S I J Whitcroft, B Lees, I F Godsland, D Crook \\ Wynn Institute for Metabolic Research, London}

Loss of ovarian function increases the incidence of cardiovascular disease in women. The effects, however, of gonadal steroid deficiency and replacement are not fully understood. One mechanism of action may be through changes in lipids and lipoproteins. In a cross sectional study of 553 healthy women with clearly defined menopausal state, we measured fasting lipids and lipoproteins. There was an age related increase in total cholesterol, triglyceride, low density lipoprotein (LDL) cholesterol, and high density lipoprotein subfraction $3\left(\mathrm{HDL}_{3}\right)$ cholesterol, and a decrease in high density lipoprotein subfraction 2 $\left(\mathrm{HDL}_{2}\right)$ cholesterol concentrations. There was also an independent increase in total cholesterol, triglyceride, and LDL cholesterol, and a decrease in $\mathrm{HDL}_{2}$ cholesterol concentration associated with the menopause. Thus loss of ovarian function appears to adversely affect lipids and lipoproteins. This may be mediated in part through changes in body fat distribution. We have previously shown that android fat correlates positively with triglyceride and LDL cholesterol, and negatively with $\mathrm{HDL}$ and $\mathrm{HDL}_{2}$ cholesterol concentrations. We have now measured regional body fat mass by dual energy $x$ ray absorptiometry in 131 healthy non-obese women. We found that total fat mass is higher in postmenopausal than in premenopausal women ( $p<0.01$ ), but there is also a significantly greater proportion of android (upper body segment) fat ( $p<0.001$ ) with a lesser proportion of gynoid (lower body segment) fat $(p<0.001)$. This redistribution of body fat would be in keeping with the adverse lipid changes found.

Thus loss of ovarian function at the menopause is associated with adverse changes in body fat distribution and lipids and lipoproteins. These changes in risk markers may in part explain the increased incidence of cardiovascular disease in postmenopausal women. 
Non-invasive monitoring of rejection: soluble interleukin- 2 receptor concentrations may predict subsequent acute allograft rejection in the transplanted heart

S H Jennison, A L P Caforio, T Fashola, S Riley, J Poloniecki, C M Corbishley, D W Holt, A J Murday, W J McKenna

Cardiac Transplant Programme, St George's Hospital, London

We have shown that endothelial major histocompatibility complex class II expression in endomyocardial biopsies predicts subsequent significant acute cardiac rejection with cellular infiltration and myocytolysis. Activation of T cells is accompanied by the elaboration of Interleukin-2 and its subsequent binding to a specific receptor complex (sIL2R). Monoclonal antibodies to sIL2R have been developed as anti-rejection agents. Studies in humans have so far failed to show a correlation between sIL2R and rejection, perhaps because of coincident infection, or liver or renal dysfunction. We analysed sIL2R concentrations at the time of biopsies, and excluded these aforementioned cases, to see if clinically significant rejection could still be predicted. Two hundred and thirty biopsies and concurrent sIL2R concentrations were obtained from 33 orthotopic heart transplant recipients in their first six postoperative months who were treated with cyclosporin, azathioprine, and prednisolone (the last was stopped after three months). Concentrations of sIL2R during acute infection, cholestasis (bilirubin $>25 \mu \mathrm{mol} / \mathrm{l}$ ), or a serum creatinine $>350 \mu \mathrm{mol} / 1$ were excluded for 98 samples. Nineteen episodes of acute rejection occurred in 18 patients. Whereas the sIL2R concentration was moderately related to the concurrent degree of rejection (ANOVA, $p=0.02$, regression $p=0.005$ ), it was more strongly related to the degree of rejection that developed 1 to 6 weeks later (ANOVA, $p=0.003$, regression $p=0.0008$ ). Expressed as a percentage change from the initial sIL2R concentration taken at the first postoperative biopsy, sIL2R strongly predicted the appearance of acute rejection one to six weeks later (regression $p=0.0001$ ).

This shows that rising sIL2R concentrations are part of a non-specific immune activation process, but may have a potential role as a predictor of clinically significant rejection in the absence of infection, cholestasis, or severe renal dysfunction.

\section{Differences in the fatty acid composition within human aortic plaques}

C V Felton, D Crook, C McCue, $M$ Davies, M F Oliver Wynn Institute for Metabolic Research, London; and British Heart Foundation Cardiovascular Pathology Unit, St George's Hospital, London

The fissuring of an atherosclerotic plaque may be a crucial event in the onset of acute myocardial infarction. The lipid composition of these complex lesions may in part determine why some plaques fissure and others remain intact. Analysis of samples from 57 non-ulcerated plaques indicated no significant difference in total saturated, monounsaturated, or polyunsaturated fatty acids between the centre and edges of these lesions. At the edge of 25 ulcerated plaques we found significant increases in the proportions of stearate, dihomo- $\gamma$-linolenate, arachidonate, and docosahexadecanoate. There was an overall increase in saturated fatty acids $(\mathrm{p}<0.001)$ and a decrease in $n-6$ polyunsaturated fatty acids $(p<0.001)$. In all plaques, the ratio of polyunsaturated:saturated fatty acids was lower at the edge compared with the centre. The fatty acids at the edge and in the centre of all plaques contained roughly $40 \%$ polyunsaturated and $35 \%$ monounsaturated fatty acids. The composition of unsaturated fatty acids in nonulcerated plaques was similar to that seen in plasma. In ulcerated plaques, however, this similarity between plaque and serum fatty acids only held for $n-3$ polyunsaturated fatty acids.

Factors such as high concentrations of relatively unstable polyunsaturated fatty acids at the periphery of advanced plaques (an area subject to considerable haemodynamic stress) and the potential formation of hydroperoxy derivatives of polyunsaturated fatty acids may help explain the prevalence of fissures at the edge of atheromatous plaques.

Improved cerebral protection with alpha-stat acid base regulation after coronary artery bypass surgery

R L Patel, D N James, D J Chambers, S Newman, G E Venn

Departments of Cardiothoracic Surgery and Cardiac Surgical Research, Rayne Institute, St Thomas's Hospital, London

It is well recognised that after coronary artery bypass surgery a variable degree of postoperative cerebrocortical dysfunction occurs. It seems likely that many of the imperfections and alterations in cerebral blood flow (CBF) during cardiopulmonary bypass (CPB) may be due to changes induced by altered acid-base management of the patien during CPB. Seventy patients undergoing elective coronary artery bypass surgery were randomised to undergo surgery using one of two differing, but widely practised, $\mathrm{CPB}$ acid base protocols; that of alpha-stat and pH-stat. CBF was measured during surgery using xenon-133 isotope clearance technique. Cerebral oxygen metabolism was measured as the cerebral metabolic rate for oxygen $\left(\mathrm{CMRO}_{2}\right)$ and matching of cerebral blood flow to oxygen demand by the brain as the cerebral extraction ratio for oxygen $\left(\mathrm{CERO}_{2}\right)$. The patients were investigated at six weeks after surgery for changes in neuropsychological function. During hypothermic $\left(28^{\circ} \mathrm{C}\right) \mathrm{CPB}$, cerebral blood flow was significantly $(\mathrm{p}<0.001)$ greater in the $\mathrm{pH}$-stat group (41 ml $\mathrm{ml}^{-1} 100 \mathrm{~g}^{-1} \mathrm{~min}^{-1} ; 95 \%$ CI 39 to 43 ) than the alpha-stat group $\left(24 \mathrm{ml}^{-1} 100 \mathrm{~g}^{-1} \mathrm{~min}^{-1} ; 95 \% \mathrm{CI} 22\right.$ to 26). The values for the $\mathrm{CERO}_{2}$ indicated a degree of mismatch of cerebral perfusion and demand during CPB in both alpha and pH-stat groups $(0.25,95 \% \mathrm{CI} 0.22$ to 0.28 and $0 \cdot 12,95 \%$ CI $0 \cdot 11$ to $0 \cdot 14$ respectively). This mismatch was far more pronounced in the $\mathrm{pH}$-stat group than the alpha-stat group, indicative of a greater degree of disruption in cerebral autoregulation in the $\mathrm{pH}$-stat group. Neuropsychological assessment showed a significantly (Fisher's exact test, $\mathrm{p}=0.02$ ) higher proportion faring less well at six weeks in the $\mathrm{pH}$-stat group than in the alpha-stat group $(17 / 35,48 \cdot 6 \% ; 95 \%$ CI 32 to $65 \cdot 1 \%$, and $7 / 35,20 \%$; $95 \%$ CI 6.7 to $33.2 \%$ respectively).

The alpha-stat managed group displayed less disruption of cerebral autoregulation during cardiac surgery than the $\mathrm{pH}$-stat group. This was accompanied by a reduction in the incidence of postoperative cerebral dysfunction. This study has shown that acid base management is an important consideration during cardiopulmonary bypass in terms of cerebral impairment after coronary artery bypass surgery. 


\section{Inhibition of thrombus formation in vivo by urokinase linked to a monoclonal antibody which recognises damaged endothelium}

M J Underwood, S Pringle, D P de Bono Department of Cardiology, Glenfield General Hospital, Leicester

Saphenous vein grafts are subject to early thrombotic occlusion, a process related to endothelial injury incurred during vein harvesting. A monoclonal antibody (P14G11) that binds to damaged endothelial cells has been linked to urokinase. This conjugate was investigated in vivo. Wistar rats were anaesthetised, a $2 \mathrm{~cm}$ segment of vena cava dissected free, and the endothelium damaged by clamping for five minutes. Test substances were introduced for 15 minutes. After 30 minutes the cava was opened and the thrombus weighed. Test substances were saline (control), urokinase $(200 \mathrm{IU} / \mathrm{ml})$, IgG-urokinase conjugate $(200 \mathrm{IU} / \mathrm{ml})$, and P14G11 antibody-urokinase conjugate $(200 \mathrm{IU} / \mathrm{ml})$. Mean weights of thrombus (mg (SE)) were: group 1, control $(\mathrm{n}=8) 17.6(2.8) ;$ P14G11-urokinase $(\mathrm{n}=8) 4.2(1 \cdot 2)$; urokinase $(\mathrm{n}=8) 16.3(3.7)$. Group 2 control ( $n=7) 14.4$ (3.8); IgG-urokinase $(n=7) 16 \cdot 1$ (4.7). The P14G11 conjugate reduced thrombus formation $v$ control and urokinase groups ( $p<0.02$ (Wilcoxon test)). No difference was found between the IgG conjugate and control groups.

The P14G11 conjugate may have a role in preventing local thrombosis in vein grafts after coronary artery bypass grafting.

\section{Pacing thresholds of epicardial electrodes}

P Kallis, N Batrick, F Bindi, A Chatzis, B E Keogh, J Mascaro, A J Murday, D J Parker, E E J Smith, $T$ Treasure

St George's Hospital, London

Failure of epicardial wires after cardiac surgery is potentially hazardous. We compared the variation of pacing thresholds in 60 patients using two commercially available pacing wires at different epicardial sites after coronary surgery with either cardioplegia or ventricular fibrillation. In 30 patients a bare, braided pacing wire (DW) was placed on the right ventricle and a medtronic localised epicardial electrode (MW) on each ventricle. In another 30 patients the DW wire was placed on the right atrium and an MW electrode was placed on each atrium. Pacing thresholds were measured at the time of placement, and at one, six, and 12 hours and daily for five days. The pacing thresholds at implantation were (mean volts (SD)) MW $0.65(0.48)$ and DW $1.28(1.0)$, in the atria and MW $0.63(0.53)$ and DW $0.93(0.45)$ in the ventricles. On the fourth postoperative day the pacing thresholds were $\mathrm{MW}$ $1.04(0.49)$ and DW $2.33(1.57)$ in the atria and MW 1.19 $(1.21)$ and DW $2.08(1 \cdot 15)$ in the ventricles. These changes in pacing thresholds were analysed by repeated measures analysis of variance which confirmed that the thresholds of both types of wire increased significantly with time ( $\mathrm{p}<0.001)$, but this deterioration was more pronounced with the braided wire both on the atrium $(p<0.005)$ and the ventricle $(p<0.05)$. The pacing threshold patterns were not affected by chamber side (left or right) or mode of myocardial preservation. The braided ventricular wire failed to capture after 24 hours in $9 / 30$ patients whereas the epicardial electrode captured in all instances $(p<0.001)$.
There was no significant difference in failure rate between the two wires in the atrial position.

Localised epicardial electrodes have consistently lower pacing thresholds than the braided wires on both the atria and ventricles and are less prone to failure on the ventricles.

\section{Early experience with intravenous oxygenation}

\author{
P Kallis, N M Al-Saady, E D Bennett, T Treasure \\ St George's Hospital, London
}

We have used intravenous oxygenation in eight patients (four men) with acute respiratory failure (acute respiratory distress syndrome four, pneumonia three, and fibrosing alveolitis one). Their median age was 39 (range 14 to 76 ). At the time of referral for intravenous oxygenation all patients were on inotropic support, five had pneumothoraces and two were on haemofiltration. Ventilatory data were: ventilated for 2 to 14 days (median six), $\mathrm{FiO}_{2} 70$ to $100 \%$ (100), positive inspired pressure 34 to $95 \mathrm{~cm} \mathrm{H}_{2} \mathrm{O}$ (49), positive end expiratory pressure 5 to $15 \mathrm{~cm} \mathrm{H}_{2} \mathrm{O}$ (12), minute volume 7 to $29 \mathrm{dl} / \mathrm{kg} \mathrm{min}(15), \mathrm{PaO}_{2} 5.4$ to $26 \mathrm{KPa}$ $(8 \cdot 5)$, and $\mathrm{PaCO}_{2} 3.7$ to $23 \mathrm{KPa}(8)$. The device was inserted via the femoral vein and positioned in the right atrium and both venae cavae. Intravenous oxygenation was used for six hours on 12 days (4), the $\mathrm{O}_{2}$ transfer achieved was 68 to $140 \mathrm{ml} / \mathrm{min}(85)$ and the $\mathrm{CO}_{2}$ removed was 40 to $106 \mathrm{ml} / \mathrm{min}(59)$. It was possible to decrease the ventilator settings with a significant increase in the $\mathrm{PaO}_{2}$ by 48 hours ( $p<0.05$ by analysis of variance) but the fall in $\mathrm{PaO}_{2}$ did not reach significance. Two patients survived and six died from multisystem failure with three being hypoxic at the time of death. Five patients had post mortem examinations and no complications related to intravenous oxygenation occurred.

Intravenous oxygenation is not at present as efficient as extra corporeal membrane oxygenation but does not have the detrimental effects on the mechanisms of coagulation that preclude the use of extra corporeal membrane oxygenation for more than a few days.

\section{Effect of continuous warm blood cardioplegia versus crystalloid cardioplegia in the isolated blood perfused rat heart}

Y Qiu, M Galinanes, D J Hearse

Cardiovascular Research, The Rayne Institute, St Thomas's Hospital, London

Continous warm blood cardioplegia (CWBC), by avoiding hypothermia and ischaemia, has been suggested to improve the protection of myocardium during cardiac surgery. With the isolated blood perfused rat heart, we compared the protective effects of CWBC with those of continuous warm $\left(37^{\circ} \mathrm{C}\right)$ crystalloid cardioplegia and the hypothermic $\left(20^{\circ} \mathrm{C}\right)$ multidose crystalloid cardioplegia. Three groups of heart ( $n=6 /$ group) were arrested with: (a) CWBC (mixture of rat blood with Fremes' solution in a ratio of 4:1) infused at $37^{\circ} \mathrm{C}$ at the rate of $0.8 \mathrm{ml} / \mathrm{min}$ throughout the period of arrest; $(b)$ multidose St Thomas's cardioplegic solution No 1 infused at $20^{\circ} \mathrm{C}$ every $30 \mathrm{~min}$ for two min; and $(c)$ continuous warm $\left(37^{\circ} \mathrm{C}\right)$, glucose enriched, crystalloid cardioplegic solution (mixture of Krebs' buffer with Fremes' solution in a ratio of 4:1) infused at 
$0.8 \mathrm{ml} / \mathrm{min}$. After three hours of arrest, the hearts were reperfused at $37^{\circ} \mathrm{C}$ with arterial blood from a support rat at a constant flow rate of $2.5 \mathrm{ml} / \mathrm{min}$. After $40 \mathrm{~min}$ of reperfusion, the perfusion pressure (an index of coronary resistance) was $60(6), 50(5)$, and $88(9) \mathrm{mm} \mathrm{Hg}$ in groups $(a),(b)$ and $(c)$ respectively. The left ventricular developed pressure (LVDP) and end diastolic pressure (LVEDP) were assessed at a range of left ventricular volumes (30 to $300 \mu \mathrm{l}$ ) with an intraventricular balloon. At a ventricular volume of $210 \mu \mathrm{l}$, the LVDP was 104 (5), 74 (3), and 64 (7) $\mathrm{mm} \mathrm{Hg}$ in groups $(a),(b)$, and $(c)$ respectively; and the LVEDP was 18 (3), 9 (2), and 33 (7) $\mathrm{mm} \mathrm{Hg}$, respectively. These results indicated that CWBC provided a greater protection of systolic function than the other two groups. The ventricular compliance was best preserved in hearts receiving hyperthermic St Thomas's Hospital cardioplegic solution No 1 .

The worst preservation of ventricular compliance and coronary vasculature were seen in the heart receiving continuous warm crystalloid cardioplegia.

Role of internal thoracic artery in patients undergoing urgent and semi-urgent surgery for complete coronary revascularisation

\section{R M Eloakley, W H Turkie, D J M Keenan}

University Department of Cardiothoracic Surgery, Manchester Royal Infirmary, Oxford Road, Manchester

Ninety eight patients aged 29 to 86 (mean 58 ) underwent coronary artery bypass grafting on an urgent or semiurgent basis for unstable angina $(64.3 \%)$, evolving infarction (19.4\%), critical left main coronary artery (LMS) stenosis ( $>90 \%$ narrowing) $(13.3 \%)$, and failed angioplasty $(3.1 \%) ; 11.2 \%$ had had previous coronary artery bypass grafting. Preoperative angiography showed significant disease ( $>50 \%$ narrowing) in the left main coronary $(17.3 \%)$, left anterior descending (95.9\%), circumflex $(84.7 \%)$, and right coronary artery $(82.7 \%)$; $78.8 \%$ had impaired left ventricular function (ejection fraction $<50 \%$ ). Of these $11 \%$ had an ejection fraction of $<30 \%$. The number of grafts ranged between one and 10 (mean six) and $90.8 \%$ had at least one internal mammary artery (of these, $32.8 \%$ had bilateral internal mammary arteries); $9 \cdot 2 \%$ received vein grafts only, seven of whom were haemodynamically unstable, and in two the internal mammary arteries were unsuitable; $13.3 \%$ had an additional cardiac procedure. Early mortality was $3.1 \%$ (95\% confidence intervals $(0.64-8.69)$ ) (two were cardiac related). Five had electrocardiographic evidence of perioperative infarction. All patients were reviewed at six weeks. Two had recurrent angina; $82.7 \%$ were in New York Heart Association functional class I, 12.2\% in class II, and $2 \%$ in class III.

Provided patients are not in cardiogenic shock, single or bilateral internal mammary arteries with their known long term advantages can be used in patients undergoing urgent complete revascularisation with an acceptable mortality and morbidity.
Effect of propafenone and verapamil on the inward calcium current in isolated cardiac myocytes

\section{J S Gill, F Lu, W J McKenna, A J Camm}

Department of Cardiological Sciences, St George's

Hospital Medical School, London

Propafenone is a potent class $1 \mathrm{c}$ agent for the treatment of supraventricular and ventricular arrhythmias. Its major action is on the fast inward sodium current with reduction of the maximal rate of depolarisation of the action potential. There is increasing evidence that slow action potentials and afterdepolarisations, both of which are dependent upon calcium entry, have important roles in arrhythmogenesis. Experiments on multicellular cardiac preparations have suggested that propafenone may block calcium currents in the myocardium. This study examines the effects of propafenone on the inward calcium current $\left(I_{C a}\right)$ in isolated cardiac myocytes and compares these with the classical calcium channel antagonist, verapamil. Single guinea pig ventricular cardiac myocytes were obtained by enzymatic digestion. Calcium currents were studied using the whole cell clamp method (external solution composition (mM) $\mathrm{NaCl} 112, \mathrm{NaHCO}_{3} 24, \mathrm{NaH}_{2} \mathrm{PO}_{4} 1$, $\mathrm{KCl} 5 \cdot 4, \mathrm{MgCl}_{2}, 1$ HEPES 5, glucose $10 ; \mathrm{pH} 7 \cdot 4$ with $\mathrm{NaOH}$ : pipette solution $\mathrm{CsCl} 130$, HEPES $15, \mathrm{MgCl}_{2} 1$, $\mathrm{Na}_{2} \mathrm{ATP}_{2} 2$, EGTA $1 ; \mathrm{pH} 7.2$ with $\mathrm{CsOH}$ ). Sodium currents were inhibited by a $100 \mathrm{~ms}$ prepulse from -80 to $-40 \mathrm{mV}$ and potassium currents by intracellular caesium. Known concentrations of drug were added by perfusion into the recording bath. Propafenone inhibited the $\mathrm{I}_{\mathrm{C}}$ over 10 to 20 minutes in these myocytes. The dose response curve was measured at 10 different concentrations of propafenone from $1 \times 10^{-8} \mathrm{~mol} / 1$ to $1 \times 10^{-3} \mathrm{~mol} / 1(\mathrm{n}=3$ to 11 cells (mean $=5$ ) for each dose). The concentration of propafenone for $50 \%$ maximal block of $I_{\mathrm{Ca}}$ was $1.5 \times 10^{-6} \mathrm{~mol} / \mathrm{l}$. Propafenone did not exhibit use dependent block of the calcium current. Propafenone at concentrations of $1 \times 10^{-6} \mathrm{~mol} / 1$ blocked the $I_{C a}$ by $42 \cdot 1$ $(4 \cdot 7) \%$ (SD) $(n=11)$ whereas verapamil at a dose of $1 \times 10^{-6} \mathrm{~mol} / 1$ blocked the $\mathrm{I}_{\mathrm{Ca}}$ by $48.6(7 \cdot 7) \%(\mathrm{n}=8)$. Verapamil showed both tonic and use dependent block of the $\mathrm{I}_{\mathrm{Ca}}$.

Propafenone is a powerful calcium channel blocker at clinically therapeutic concentrations of the drug; its mechanism of action, however, differs from that of verapamil.

Naloxone has non-receptor mediated Class I and Class III antiarrhythmic activity and attenuates the rate of extracellular potassium accumulation during myocardial ischaemia

K G Oldroyd, M N Hicks, $S$ M Cobbe

Department of Medical Cardiology, Glasgow Royal Infirmary

Opioid peptide release during myocardial ischaemia may contribute to early ischaemic arrhythmias. Naloxone and other opioid receptor antagonists possess antiarrhythmic activity in animal models of coronary artery occlusion but this may be due to non-receptor mediated effects rather than opioid receptor blockade. We have investigated the cardiac electrophysiological effects of naloxone in isolated 
myocardium from New Zealand White rabbits. In superfused papillary muscles, naloxone $(n=7)$ produced a dosedependent parallel prolongation of action potential duration (APD) and effective refractory period (ERP), a class III effect. Mean (SEM) values were 216 (11) $\mathrm{ms}$ and 210 (11) ms respectively at baseline, $240(14) \mathrm{ms}$ and 232 (13) $\mathrm{ms}$ at $10^{-6} \mathrm{~mol} / \mathrm{1}^{-1}(\mathrm{p}<0.001)$, and $327(8) \mathrm{ms}$ and $320(10) \mathrm{ms}$ at $10^{-4} \mathrm{~mol} / 1^{-1}(p<0.00001)$. Similar effects were seen with another opioid antagonist (nalmafene $n=7$ ) and an agonist (morphine $n=7$ ). Under hyperkalaemic conditions, naloxone $(n=7)$ reduced the maximum upstroke velocity of the action potential $\left(\mathrm{dV} / \mathrm{dt}_{\max }\right)$ and produced postrepolarisation refractoriness, a class I effect. In arterially perfused interventricular septa, both dl-naloxone (active at opioid receptors $n=7$ ) and $d$-naloxone (inactive $n=6$ ) significantly prolonged APD and ERP compared with control $(n=7)$. During global ischaemia the class III effects were gradually lost but the shortening in APD was more marked than the reduction in ERP leading to the development of postrepolarisation refractoriness. Both compounds reduced the accumulation of extracellular potassium: control $0.28(0.01) \mathrm{mmol} / \mathrm{1}^{-1}$ $\min ^{-1}, \mathrm{~d}$ l-naloxone $0.18(0.01) \mathrm{mmol} / \mathrm{l}^{-1} \mathrm{~min}^{-1}$, and $\mathrm{d}-$ naloxone $0.20(0.02) \mathrm{mmol} / 1^{-1} \mathrm{~min}^{-1}, \mathrm{p}=0.003$. The fall in $\mathrm{dV} / \mathrm{dt}_{\max }$ during ischaemia was enhanced compared with control.

Attenuation of the rate of rise of extracellular potassium concentration during ischaemia and non-opioid receptor mediated cardiac electrophysiological effects may explain the antiarrhythmic activity of naloxone.

\section{Stretch-induced electrical dispersion: evidence in humans from a new in vivo model}

\section{P Taggart, P M I Sutton, R John, M J Lab, S Horner, R H Swanton \\ Middlesex Hospital, UCMSM and Charing Cross \\ Hospital, London}

Recent experimental evidence has highlighted the importance of diastolic stretch in the initiation of ventricular arrhythmias resulting from mechano-electric interacrion. We have developed a model for the study of this mechanism in humans using the presence or absence of the atrial contribution to ventricular filling to alter diastolic stretch in the human left and right ventricle. Duration of monophasic action potential was measured simultaneously from the left and right ventricular endocardium as a measure of repolarisation and dispersion of repolarisation between the two regions. Seventeen patients were studied (aged to 38 to 69, median 49). After routine coronary angiography, the two monophasic action potential catheter electrodes and two pacing electrodes were positioned (right atrium and right ventricle). In a total of 34 observations, changing from steady state right ventricular pacing to atrioventricular sequential pacing (thereby incorporating the atrial contribution to ventricular filling) raised peak systolic pressure from 112 (2) (mean (SEM)) $\mathrm{mm} \mathrm{Hg}$ to 142 (2). The right ventricular action potential duration changed in 27 observations ( $p<0.02$ ) by up to $20 \mathrm{~ms}$ and the left ventricular action potential changed by a maximum of $42 \mathrm{~ms}(n=28 ; p<0.025)$. The overall difference in duration of monophasic action potential between the two sites (that is, the electrical dispersion between the two regions) altered by a median of $12 \mathrm{~ms}$ (ranges: $0-4 \mathrm{~ms}$ $(n=9), 6-10(n=6), 12-20(n=14), 20-42(n=5))$.

Our results have shown that alteration in diastolic stretch using the atrial component to ventricular filling alters duration of action potential at both right and left ventricular sites and dispersion between the two regions. Thse findings are consistent with mechano-electric feedback whereby alteration in ventricular loading (diastolic stretch) alters the duration of the action potential (and hence refractoriness). The model that we have employed is applicable to the study of stretch induced arrhythmogenic mechanisms in patients with coronary heart disease.

Clinical and haemodynamic performance of the $19 \mathrm{~mm}$ Carpentier-Edwards supra-annular aortic valve

P Kallis, J F Sneddon, L A Simpson, A Fung,

J R Pepper, E E J Smith

St George's Hospital, London

The clinical and haemodynamic performance of the $19 \mathrm{~mm}$ Carpentier-Edwards supra-annular aortic valve (CE-SAV) is unknown compared with the larger valves. Over four years we implanted $19 \mathrm{~mm} \mathrm{CE-SAV}$ into 21 patients (20 women), mean age $75(1.2)$ (range 59-86) and mean body surface area $1.6(0.03) \mathrm{m}^{2}$ (range 1.3 to 1.7 ). There were four deaths, one at surgery and three late non-cardiac deaths. Follow up of the 17 survivors for a mean of 20 (3.1) months (range 2-42) found symptomatic improvement in all 17 (all are now in New York Heart Association functional class I and II). There were no valve related complications and no patient required long term anticoagulation. Echocardiographic and Doppler studies were used to assess the in vivo haemodynamic profile of the valve. Mean postoperative aortic valve gradient was $34 \cdot 1$ (2.7) $\mathrm{mm} \mathrm{Hg}$ (range 19-52). Functional valve orifice area was $1 \cdot 1(0.09) \mathrm{cm}^{2}$ (range $\left.0 \cdot 6-1 \cdot 8\right)$. Mean cardiac output was $3.92(0.17) 1 / \mathrm{min}$ (range $3 \cdot 2-5 \cdot 1)$ with a mean cardiac index of $2.5(0 \cdot 11) 1 / \mathrm{min} / \mathrm{m}^{2}$ (range $\left.2 \cdot 1-3 \cdot 2\right)$.

Aortic valve replacement with the $19 \mathrm{~mm} \mathrm{CE}-S A V$ has a low operative mortality and offers major clinical benefits despite moderate transprosthetic gradients. This approach provides an alternative management strategy in elderly patients who would otherwise require low profile mechanical valves or aortic root enlargement.

\section{Ambulatory pulmonary artery pressure monitoring in pulmonary hypertension: diurnal variation and effects of exercise and daily activities}

J S R Gibbs, H Purcell, A Sullivan, C Wright, J Sparrow, K Fox, M Yacoub

Royal Brompton National Heart and Lung Hospital, London

Ambulatory pulmonary monitoring was used to investigate the pulmonary haemodynamics of primary pulmonary hypertension in six patients. These patients (age 38, range 17-54) who were awaiting heart-lung transplantation underwent 24 hour ambulatory pulmonary artery pressure recordings with a micromanometer tipped catheter incorporating an in vivo calibration system, and a digital recorder. During this period patients undertook submaximum exercise walking up and down stairs, and walking on the level, and maximum exercise on a treadmill. Four of these patients underwent simultaneous non- 
invasive ambulatory systemic blood pressure recording. The mean 24 hour pulmonary artery pressure $(\mathrm{mm} \mathrm{Hg}$ (SD)) was 101 (25)/47 (12). The mean day time pressure was $99(24) / 47$ (12) and mean night time pressure was 107 (28)/50 (14). From lying to standing the pressure fell from 106 (33)/45(14) to 81(26)/37 (15.9). At peak exercise walking on the level the pressure was 127 (39)/63 (18), on the stairs $129.5(39) / 56(16)$, and on the readmill $136(35) / 62$ (17). Patients rated the stairs as the most severe exercise. Although pulmonary artery pressure appeared primarily to be related to the patients' activities, two patients sustained an unprovoked episode of systolic pressure exceeding 200 $\mathrm{mm} \mathrm{Hg}$ lasting about one minute. Blood pressure monitoring showed that the 24 hour mean was not significantly different from pulmonary artery pressure. The mean day time blood pressure was $101(23) / 68(16)$ and the mean night time pressure was $94(16) / 62(9)$. At peak exercise the blood pressure walking was $85(34) / 69(14)$, on the satirs 88 (34)/65 (17), and on the treadmill 95 (30)/59 (14).

Pulmonary artery pressure was at systemic level and tended to rise at night to the same level as lying during the day. Pulmonary artery pressure rose on exercise to a level at which there was no significant difference between submaximum and maximum exercise. Pulmonary artery pressure became suprasystemic at night and on exercise, and was associated with a fall in blood pressure. These findings may have implications with regard to the mechanisms involved in regulating pulmonary artery pressure in these patients as well as therapeutic implications.

\section{Role of transoesophageal echocardiography in cardiac surgical intensive care}

\author{
A C H Pell, C Campanella, S Prasad, A R J Cale, \\ A J Duncan, G R Sutherland \\ Departments of Cardiac Surgery and Cardiology, \\ Western General Hospital, Edinburgh
}

Transoesophageal echocardiography (TEE) is becoming established as a valuable intraoperative procedure. Its role, however, in the cardiac surgical intensive care unit is less well defined. To investigate this we reviewed all TEE studies performed in a cardiac surgical intensive care unit over a six month period $(n=50)$. Indications for emergency TEE were suspected prosthetic valve dysfunction $(n=5)$, intracardiac source of embolism $(n=2)$, or aortic pathology $(n=3)$, unexplained postoperative hypotension/ cardiac failure $(n=10)$, and major thoracic trauma $(n=7)$. TEE was only performed if precordial echocardiography had failed to give a definitive diagnosis. In 23 patients, intraoperative TEE monitoring of left ventricular function during coronary artery bypass surgery was continued electively in the intensive care unit postoperatively. In thoracic trauma, TEE showed a pericardial effusion $(n=1)$, a large trapped right atrial fat embolus $(n=1)$, and significant myocardial wall motion abnormalities consistent with myocardial contusion $(\mathrm{n}=2)$, and excluded aortic dissection $(n=7)$. In unexplained postoperative hypotension or cardiac failure, TEE showed severe global impairment of left ventricular function $(n=1)$, a pericardial effusion $(n=2)$, major regional wall motion abnormalities consistent with myocardial ischaemia or infarction $(n=3)$, patch dehiscence $(n=1)$, and no abnormality $(n=3)$. In suspected prosthetic valve dysfunction, TEE showed paraprosthetic leakage $(n=3)$ and obstructed mitral prosthesis $(n=1)$, and helped exclude infective endocarditis. Neither of the patients investigated for postoperative neurological deficit had evidence of intracardiac thrombus or patent foramen ovale. In suspected aortic pathology, TEE showed a new thoracic aortic dilatation $(n=1)$ and excluded aortic dissection $(n=2)$. Elective postoperative monitoring showed a new regional wall motion abnormality in one patient with severe hypotension and persistent regional wall motion abnormalities in two patients. It also assisted in the diagnosis of hypovolaemia or impaired left ventricular function and excluded other complications in patients with moderate hypotension $(n=4)$. Thus TEE gave important diagnostic information in $24 / 27$ patients studied acutely and $7 / 23$ patients monitored electively.

TEE is a valuable diagnostic procedure in the cardiac surgical intensive care unit and may be of assistance in routine monitoring after coronary artery bypass surgery.

\section{AIDS-associated heart disease relates to opportunistic infection rather than to the HIV virus: a study of 113 consecutive patients}

\section{F Akhras, S Dubrey, C Wood, B Gazzard, M Noble Cardiovascular Department, Westminster Hospital, Page Street, London}

One hundred and thirteen consecutive homosexual men mean age 37 (range 23-57) with HIV infection were prospectively studied by Doppler echocardiography. Ninety five patients had the AIDS syndrome (group A) for 19 (12) months and 18 patients had HIV infection without opportunistic infections (group B) for 43 (28) months. Doppler echocardiography was normal in 38 patients $(40 \%)$ of group A and in 14 patients $(78 \%)$ of group B. Pericardial effusion was found in 40 patients (43\%) of group $A$ and in only two patients of group B. Left ventricular dilatation, or dysfunction, or both was found in 17 patients $(18 \%)$, aortic root dilatation and aortic regurgitation in seven patients, and an intracardiac echogenic mass in six patients $(6 \%)$ of group A, but were not documented in group B. Mitral valve prolapse, or mitral regurgitation, or both was found in 26 and two patients, isolated right ventricular dysfunction and dilatation in three and one patients of group A and group B respectively. Pneumocystis carinii occurred in $57(60 \%)$; cytomegalovirus in $32(34 \%)$; mycobacterium in $21(22 \%)$; toxoplasmosis in eight $(8 \%)$; and Kaposi sarcoma in $36(38 \%)$ patients. Forty one patients in group A had a cardiac presentation; of these nine had cardiomegaly with clinical signs of heart failure and a further nine patients had tachyarrhythmias compared with only one patient with such presentation in group B. Despite the CD4 lymphocyte count (\%) being significantly lower in group A than in group B $(5 \cdot 1(5 \cdot 4) v 15 \cdot 5(5 \cdot 8), \mathrm{p}<0.0001$, (normal 27 to 49)), statistical analysis showed that the CD4 count is not correlated to the presence of pericardial effusion, left ventricular dysfunction, right sided cardiac enlargement, or the duration of HIV infection in both groups.

Although often not diagnosed clinically, cardiac involvement in patients with AIDS is a clinical reality. Pericardial effusion, cardiomyopathy, and left ventricular dysfunction appear to have a high prevalence in male homosexual patients with AIDS. These echocardiographic abnormalities are associated with clinically apparent intercurrent infections, which appear to be the culprit for cardiac involvement, rather than the HIV virus or the severity of infection as reflected by the CD4 count. These findings have important implications for future therapeutic studies in patients with AIDS related heart disease. 


\section{Is HIV cardiomyopathy caused by selenium deficiency?}

\section{A J Jacob, G S Fell, A G Bird, R P Brettle, N A Boon Department of Cardiology, Royal Infirmary, Edinburgh; and Trace Metals Unit, Royal Infirmary, Glasgow}

Cardiomyopathy due to selenium deficiency is endemic in the Keshan district of China. It may also occur in the context of human immunodeficiency virus (HIV) infection. To test this hypothesis, we have measured serum selenium concentrations in nine HIV positive patients with cardiomyopathy and nine HIV positive controls with echocardiographically normal hearts matched for age, sex, risk group, body mass index, and CD4 count (a measure of the severity of HIV disease). Cardiomyopathy was defined as global hypokinesia of the left ventricle and fractional shortening of less than $28 \%$. Selenium assay was performed using carbon atomic absorption spectroscopy. The normal ranges for serum selenium concentrations and $\mathrm{CD} 4$ count in our laboratory are 0.86 to $1.6 \mu \mathrm{mol} / 1$ and 800 to 1500 cells $/ \mathrm{mm}^{3}$ respectively. The patients with HIV cardiomyopathy had a mean CD4 count of $25 \cdot 8(50 \cdot 3) \mathrm{cells} / \mathrm{mm}^{3}$, serum selenium concentration $0.36(0 \cdot 19) \mu \mathrm{mol} / 1$, and body mass index of $19.31(3.53) \mathrm{kg} / \mathrm{m}^{2}$. Those with normal hearts had a mean CD4 count of $40.4(40 \cdot 1)$ cells $/ \mathrm{mm}^{3}$, serum selenium concentration $0.59(0.27) \mu \mathrm{mol} / 1$, and body mass index of $19.00(2 \cdot 87) \mathrm{kg} / \mathrm{m}^{2}$. Only the selenium values were significantly different between the two groups $(\mathrm{p}<0.05)$.

Selenium deficiency is common in late stage HIV infection. Patients with HIV cardiomyopathy have a significantly lower serum selenium concentration than HIV positive patients matched for stage of disease and body mass index. This suggests that selenium deficiency may play a part in the pathogenesis of HIV related cardiomyopathy.

\section{Symmetrical and asymmetrical ${ }^{123}$ I- metaiodobenzylguanidine scans in patients with long QT syndromes}

J S Gill, N J Linken, J Gant, D E Ward, A J Camm Cardiological Science, St George's Hospital Medical School, London

The arrhythmogenic mechanism in congenital long QT syndromes has been proposed to be an imbalance of the cardiac sympathetic innervation. $\mathrm{I}^{123}$ metaiodobenzyl-guanidine (MIBG) is an analogue of noradrenaline which is taken up by the sympathetic nerve terminals and can be used for visualisation of the cardiac sympathetic nerve supply. This study examines the regional sympathetic innervation in patients with long QT syndromes and compares this with the result in normal control patients. Four patients with long QT syndromes (age range 19-55; all women QTc $>0.44 \mathrm{~s}$ in all) were studied and compared with a control group consisting of six patients with atrioventricular reentrant tachycardia and eight patients with normal coronary arteries at angiogram and no evidence of fixed or reversible ischaemia on thallium-201 scanning. Patients attended fasting, and were given 370 mbq per $1.73 \mathrm{~m}^{2}$ of body surface $\mathrm{I}^{123}$ MIBG via a periph- eral vein. SPECT gamma scanning was performed three hours later by a single pass with $45 \mathrm{~s}$ per step $(64 \times 64$ matrix). Left ventricular MIBG uptake was processed into bull's eye target plots. The inferior portion of the scan frequently exhibited artifacts due to uptake of MIBG in the liver or spleen and was not used for statistical analysis. Asymmetry of MIBG uptake was defined as the ratio of uptake in the upper quadrants (posterior:anterior) beyond the range 0.95-1.25 (derived from mean (2 SD) from the control group). None of the patients in the control group had an abnormal ratio. Of the patients with long QT syndromes, two had abnormal scans (ratios 1.31 and 1.29) suggesting decreased sympathetic supply on the septal surface of the left ventricle, the other two patients had symmetrical scans (ratios $1 \cdot 1$ and $1 \cdot 1$ ). The left cervical ganglion supplies the superior and posterior portion of the ventricle and the right cervical ganglion the anterior and septal portion.

The results of this study suggest that some patients with long QT syndromes have relative denervation in the area supplied by the right cervical ganglion, but there are patients who have completely normal scans. This suggests that asymmetry of cardiac sympathetic nerve supply may not be the sole mechanism leading to the genesis of long QT syndromes.

Evidence of asymmetrical cardiac sympathetic innervation in the Romano-Ward syndrome from studies using ${ }^{123}$ I-metaiodobenzylguanidine

M J Metcalfe, S Cross, J Davidson, K Jennings, $S$ Walton

Department of Cardiology, Aberdeen Royal Infirmary, Aberdeen

The Romano-Ward syndrome is a congenital cause of a prolonged QT/U interval and is thought to be due to an imbalance between the left and right sympathetic innervation of the heart. ${ }^{123} \mathrm{I}$-metaiodobenzylguanidine (MIBG) is an analogue of noradrenaline and is taken up into the postsynaptic nerve terminals. The distribution of the sympathetic supply to the heart can thus be assessed by single photon emission computed tomography (SPECT). Eight patients with proven normal coronary arteries and left ventricular contraction, as assessed by cardiac catheterisation, were compared with a family of three patients (mother and two daughters) with Romano-Ward syndrome. Only one of these patients was symptomatic with periodic polymorphic ventricular tachycardia, the others not exhibiting any significant dysrhythmias on either stress or ambulatory electrocardiographic monitoring. Three hour MIBG uptake was assessed both subjectively and objectively using a radial, segmental method of SPECT analysis. Both asymptomatic members of the family had a distribution of isotope comparable with the controls. The symptomatic family member had a significantly asymmetric distribution, with more activity present within the lateral wall.

This finding supports an asymmetric sympathetic innervation of the heart in patients with symptomatic Romano-Ward syndrome. 
Transcatheter occlusion of patent arterial ducts: six years' experience at Guy's Hospital

\author{
A H Tabatabaei, I C Huggon, E J Baker, S A Qureshi, \\ M Tynan \\ Guy's Hospital, London
}

Since 1986, of 147 patients in whom transcatheter occlusion of a patent arterial duct with an umbrella device was attempted, a first device was successfully implanted in $134(91 \%)$ patients. In the remaining 13 , the duct had unusual anatomy in two, was too small in six (two previously surgically ligated), and was too large in three. Abnormal systemic venous anatomy precluded passage of the introducer sheath in two. The age at implantation ranged from seven months to 67 years (median $2 \cdot 87$ years) and weight from $6 \cdot 15$ to $79.6 \mathrm{Kg}$ (median $13.7 \mathrm{Kg}$ ). Two patients had had a previous Senning operation and five had had previous duct ligation. A $12 \mathrm{~mm}$ device was implanted in 93 patients and a $17 \mathrm{~mm}$ device in 41 . A complete arteriovenous guidewire circuit was established to position the introducer sheath in seven patients. Complications of the procedure included embolisation of the device to the femoral artery in one patient and severe haemolysis needing surgical removal of the device in one patient. No case of infective endarteritis occurred. At the latest follow up colour Doppler study confirmed complete occlusion of the duct in $92(69 \%)$ patients after implantation of a single device. Of the 42 patients with residual leak 22 have follow up of less than four months. Of those patients with follow up of more than four months, 17 subsequently had attempted implantation of a second device, which was successful in $16(94 \%)$. The duct in the remaining patient could not be dilated sufficiently to allow passage of the delivery system. After a mean follow up of eight months, 13 ducts are completely occluded on colour Doppler study. Three ducts still have a small residual lead one day, six weeks, and 18 months after the second insertion.

Transcatheter occlusion of a patent arterial duct is feasible and is associated with few complications. There is an important incidence of residual leak and implantation of a second umbrella device is sometimes needed to achieve complete occlusion.

\section{Improved left ventricular subendocardial function after coronary angioplasty}

\author{
M Y Henein, S J D Brecker, H B Xiao, B B Stojnic, \\ D G Gibson \\ Cardiac Department, Royal Brompton National Heart \\ and Lung Hospital, London
}

The subendocardial fibres in human myocardium are longitudinally arranged. To assess whether their resting function is impaired by coronary artery disease, we compared 22 patients before and after successful single vessel coronary angioplasty (13 left anterior descending, two circumflex, and seven right coronary artery), with 25 age matched normal subjects. Long axis function was measured with $M$ mode echocardiography of the left (L) and septal (S) sites of mitral ring motion with simultaneous phonocardiography, together with transverse cavity dimension and wall thickness. M-mode records were digitised. Transmitral flow was assessed by pulsed Doppler. In 18/22 patients, basal long axis function was abnormal when compared with normals, with prolonged systolic shortening (L: 79 (26) ms $v 52$ (10), and S: 81 (29) ms $v 50(10)$, p values <0.05) beyond aortic closure, reduced early diastolic lengthening rates $(\mathrm{L}: 4 \cdot 8(1 \cdot 7) \mathrm{cm} / \mathrm{s}$ $v 7.7(1.9)$, and $\mathrm{S}: 4(1.7) \mathrm{cm} / \mathrm{s} v 6.7(1.8)$, $\mathrm{p}$ values $<0.05)$, and increased A wave amplitude (L:41.0 (7.0)\% total excursion $v 25 \cdot 2(6.5) \%$, and S:43.0 (7.1) v $27.5(5.9) \%$ ). After angioplasty, the duration of aortic closuremaximum shortening-fell to $57.0(21.0) \mathrm{ms}(\mathrm{L})$ and 59.0 $(23.0) \mathrm{ms}(\mathrm{S})$ (p values $<0.001$ ), early diastolic velocities increased to $5.5(1.8) \mathrm{cm} / \mathrm{s}(\mathrm{L})$ and $4.7(1.8) \mathrm{cm} / \mathrm{s}(\mathrm{S})$ (p values $<0.001$ ), and $A$ wave amplitude fell to 37.1 $(8 \cdot 2) \%$ total excursion (L) $(p<0.01)$ and $42.0(7 \cdot 1) \%(S)$ $(\mathrm{p}<0.5)$, at both sites. At the same time, left ventricular posterior wall thinning rate increased to $6.0(2.0) v 4.8(1 \cdot 7)$ $\mathrm{cm} / \mathrm{s}(\mathrm{p}<0.001)$, and the Doppler $\mathrm{E} / \mathrm{A}$ ratio rose from 0.9 $(0.3)$ to $1.1(0.3)(p<0.001)$. There was no significant difference in the rates of minor axis change.

Thus in patients requring angioplasty diastolic subendocardial function is often abnormal even in the absence of angina. These changes are promptly reversible by angioplasty, strongly suggesting that they are the direct effect of coronary stenosis. The results show the interrelations between subendocardial function, left ventricular wall dynamics, and filling pattern.

\section{Are transoesophageal ultrasound studies required to select patients for transcatheter closure of atrial septal defects?}

O Stumper, P Hoffman, G R Sutherland, M J Godman Royal Hospital for Sick Children, Edinburgh

Transoesophageal echocardiography has been shown to be a prerequisite for successful transcatheter clamshell occlusion of atrial septal defects. Its value in patient selection, however, has as yet not been assessed. We studied 38 patients (age 3 to 66 ) with the clinical or precordial ultrasound (PE) diagnosis of atrial septal defect by transoesophageal ultrasound (TEE) techniques to define its relative value in patient selection. Precordial studies included subcostal and parasternal imaging and colour flow mapping; TEE was performed using single plane equipment in 17 patients and biplane equipment in 21 . All studies were performed using a standard examination protocol. Cardiac catheterisation and angiography were carried out in 15 patients. All PE and TEE studies were analysed by two independent observers and patients were assigned to three categories: $(a)$ those who were unsuitable for clamshell occlusion, $(b)$ those who might be, and $(c)$ those who were judged to have a perfect anatomy for device occlusion. On the basis of the TEE study 11 patients were judged to be unsuitable for transcatheter closure, of whom 10 subsequently underwent surgical defect closure. Only five of these were identified by PE. Important information missed by PE in this group included sinus venosus defect in two, posterior defect in one, and multiple large defects in two. Defects with multiple fenestrations were documented by TEE in a further four patients (only 2 by PE), in whom clamshell occlusion, however, appeared to be feasible. Deficiency of the anterosuperior margin of the defect (eight patients), which may preclude effective occlusion of the defect, was better documented by TEE than by PE. Optimal anatomy for device occlusion was suggested by 17 of the 31 patients in whom adequate quality images were obtained. This was confirmed by TEE in 13 and in another three patients in whom PE remained ambiguous. 
Thus TEE imaging allows a comprehensive tomographic assessment of atrial septal defects and their relation to near by structures. In children PE allows for adequate patient selection in the majority of cases. In older children, adolescent, and adult patients TEE appears to be the technique of choice for patient selection; TEE should be performed in all patients in whom PE gives inadequate images or cannot show fully the entire defect margin and its relation to other structures.

\section{Physical activity, hypertension, and risk of heart attack}

A G Shaper, G Wannamethee, $M$ Walker

Royal Free Hospital School of Medicine, London

Physical activity is associated with a reduced risk of heart attack. Several studies have shown, however, that at vigorous levels of physical activity in middle aged men there may be an increase in the risk of heart attack above the level seen in moderate or moderately vigorous men. This phenomenon has been found in the British Regional Heart Study and the data have now been explored to determine possible mechanisms for this unexpected finding. The group comprises 7735 men aged 40 to 59 years drawn from one general practice in each of 24 towns. The men were classified into six broad categories based on their usual reported activity: inactive, occasional, light, moderate, moderately vigorous, and vigorous. All men were followed up for 9.5 years for cardiovascular morbidity and all cause mortality. Men without evidence of pre-existing ischaemic heart disease were separated into normotensive $(n=3888)$ and hypertensive $(n=1806)$ groups, ie systolic $B P>160$ $\mathrm{mm} \mathrm{Hg}$ or diastolic BP $>90 \mathrm{~mm} \mathrm{Hg}$ or on regular antihypertensive treatment. In normotensive men there was an inverse relation between physical activity and heart attack. In hypertensive men there was a decline in risk to levels of moderate and moderately vigorous activity followed by an increase in risk in the vigorously active group. When other risk factors were taken into account, the increased risk of heart attack in vigorously active hypertensive men was more pronounced than before such adjustment. The phenomenon was most apparent in the younger (40 to 49 year old) men.

Analysis of interactions between risk factors suggests that hypertension was the critical factor in determining the increased risk of heart attack in vigorously active younger middle aged men. These findings are highly relevant to the current promotion of physical exercise in the management of hypertension.

\section{Alcohol and sudden cardiac death}

G Wannamethee, A G Shaper

Royal Free Hospital School of Medicine, London

Heavy drinking may induce cardiac arrhythmias and ventricular tachycardia, mechanisms associated with sudden cardiac death. The association, however, between heavy drinking and the risk of sudden cardiac death in the general population is uncertain and reported findings have been inconsistent. The relation between alcohol and sudden death from ischaemic heart disease has been examined in a large prospective study involving 7735 middle aged British men drawn from general practices in 24 towns (The British
Regional Heart Study). During the follow up period of eight years there were 217 deaths from ischaemic heart disease of which $117(54 \%)$ were classified as sudden (death occurring within one hour of the onset of symptoms). Although heavy drinkers ( $>6$ drinks/day) did not show high incidence rates of death from heart attacks, they showed the highest rates of sudden cardiac death. Nondrinkers showed the lowest rates. Deaths which occurred in heavy drinkers were predominantly sudden $(75 \%)$ and this was seen in all men, irrespective of the presence or degree of pre-existing ischaemic heart disease. The increased incidence of sudden death in heavy drinkers was most apparent in men without pre-existing evidence of ischaemic heart disease, with heavy drinkers showing a $60 \%$ increase in risk compared with occasional or light drinkers even after adjustment for age, social class, and smoking. In men free of ischaemic heart disease, heavy drinkers had marginally significantly higher incidence rates of sudden death than all other drinkers combined $(R R=2.00,95 \%$ CI $0.98-4 \cdot 8, p=0.06)$ after adjustment for age, social class, and smoking. Further adjustment for systolic blood pressure reduced the risk to $1 \cdot 7$.

This study suggests that heavy drinking is associated with an increased risk of sudden death, possibly due to a direct effect of alcohol on the myocardium.

\section{Bovine internal mammary artery grafts: do they remain patent?}

I M Mitchell, A R Essop, P J Scott, P Martin, N R Saunders, R U Nair, G J Williams

Killingbeck Hospital, Leeds

A successful outcome after coronary artery bypass surgery depends largely upon the choice of graft conduit. It is well established that the best long-term results are obtained with internal mammary artery grafts but autologous saphenous vein and the gastroepiploic artery are useful alternatives. Other conduits such as cephalic veins and synthetic grafts have proved grossly inferior. With increasing numbers of patients requiring reoperation plus a proportion of new patients presenting with insufficient or poor quality saphenous veins, there is a need to find a more suitable conduit that is readily available, easy to handle, and has good long-term patency. To this end, bovine internal mammary artery grafts have been developed. We have used a total of 26 such grafts in 18 patients since January 1990. There were 13 men and five women, men age 61 (range 52 to 71 ). Two patients died, one at 10 months, due to cardiac failure and the other at four months, after myocardial infarction. Postoperative angiography has been performed in 11 of the other 16 patients, at a mean of 10 months after surgery (range 3 to 23 months). Of 15 bovine internal mammary artery grafts implanted and restudied, three are currently patent $(20 \%)$. We report the results of clotting studies and analysis of lipid state. These patients do not appear to represent an atypical group, either in terms of coagulopathy, native coronary artery size, or the type of vessel disease.

This is the largest experience and longest follow up of bovine internal mammary artery grafts so far reported. Our poor results, however, contrast with the early enthusiasm from other centres. 
Impaired myocardial endothelial and non-endothelial dependent microvascular function in patients with coronary occlusive disease after cardiac transplantation

\section{P A Mullins, A Chauhan, S R Large, J Wallwork, P M Schofield \\ Transplant Unit, Papworth Hospital, Cambridge}

Coronary occlusive disease is the major long-term problem after cardiac transplantation. It is a diffuse disease which also affects smaller coronary vessels and is difficult to assess angiographically. Coronary flow measurements allow investigation of vasodilatory responses in the coronary microvasculature. We investigated the hypothesis that coronary flow responses (CFR) to papaverine (a nonendothelial dependent vasodilator) and acetylcholine (an endothelial dependent vasodilator) are impaired in patients with coronary occlusive disease after cardiac transplantation. The CFR was assessed in 27 cardiac transplant patients with normal coronary anatomy (group 2) and 20 patients with evidence of mild coronary occlusive disease on angiography (mean \% stenosis diameter 23 (SD 6)\%) in a proximal coronary vessel (group 1), with 12 of these in the left anterior descending coronary artery (mean \% stenosis diameter $24($ SD 8$) \%$ ). A Doppler flow probe was inserted into the proximal left anterior descending coronary artery in each patient. Incremental doses of intracoronary papaverine (pap), and glyceryl trinitrate (GTN) followed by acetylcholine (Ach) were given until maximum hyperaemia was achieved. The CFR for each drug was defined as the ratio of resting (RFV) to peak coronary blood velocity. The CFR after papaverine was impaired in group 1 compared with group 2 patients, $(2.5(1.0) v 4.2(1.0) ; \mathrm{p}<0.001))$. The CFR after Ach was impaired in group 1 patients compared with group $2,(1.8(0.8) v 2.9(0.9) ; \mathrm{p}=0.04))$. There was no difference in RFV after GTN (group 1 RFV 6.9 $(5 \cdot 0)$ before and $6.9(6.0) \mathrm{cm} / \mathrm{s}$ after GTN; group $2 \mathrm{RFV} 7 \cdot 4$ $(4 \cdot 7)$ before and $7 \cdot 3(5 \cdot 5) \mathrm{cm} / \mathrm{s}$ after $\mathrm{GTN})$, or in diameter of left anterior descending coronary artery between groups after the vasodilatory drugs.

Coronary endothelial and non-endothelial dependent microvascular responses are impaired in cardiac transplant patients with minor proximal coronary stenoses. This disturbance of cardiac microvascular function may contribute to the late morbidity and mortality seen in cardiac transplant patients with coronary occlusive disease.

\section{Gated F18 fluorodeoxyglucose positron emission tomography: a novel approach for the detection of viable myocardium}

\section{H S Lee, S J Cross, M Norton, S Walton Departments of Cardiology and Medical Physics, Abedeen Royal Infirmary, Aberdeen}

Detection of viable myocardium is important and should aid the decision making in coronary artery revascularisation and cardiac transplantation. A novel approach to detect regions of viable myocardium using gated fluorodeoxyglucose (FDG) positron emission tomography is described. This directly measures cardiac motion simultaneously with regional uptake of FDG. The alternative double isotope methods (FDG + perfusion imaging) increases the patient radiation dose and cannot take account of any alteration in myocardial characteristics occurring in the period between studies. Thirty six patients were studied consisting of 27 patients (mean age (SD) 58 (7.8); 22 men with chronic coronary artery disease and abnormal left ventricular contraction documented by cardiac catheterisation. Eight patients (mean age $55(7 \cdot 1)$; three men) with normal coronary arteries, left ventriculogram, and exercise electrocardiogram acted as controls. One patient had dilated cardiomyopathy. Patients were glucose loaded. Decay compensated scans were performed at six levels, $16 \mathrm{~mm}$ apart to cover the left ventricle. Data collection was gated into eight phases with the three dimensional matrix resliced into eight short axis sections for each phase. Radial shortenings were obtained at 32 angles for each short axis section. The left ventricular conformation was thus defined by $32 \times 8$ radial shortening distances. Conformational changes of radial shortenings and regional uptake of FDG were displayed using a bull's eye presentation. Cine loop images can also be viewed at any section or orientation. Viable myocardium was defined as a region of akinetic myocardium with good FDG uptake (FDG $\geqslant$ normal myocardial region). Viable myocardium was detected in three patients, one in the inferolateral, one in the anterior, and one in the anteroseptal region. Another study of a patient with dilated cardiomyopathy further validated the technique showing good global uptake of FDG but reduced contraction. Left ventricular angiography confirmed these regions of akinesis.

Gated FDG positron emission tomography can simultaneously detect regions of metabolically active myocardium with poor contraction using a single scan: these regions are potentially viable.

\section{Post-infarct septal rupture in the elderly}

G Parry, J Goudevenos, D S Reid, P C Adams Department of Cardiology, Freeman Hospital, Newcastle upon Tyne

Age is often used as a discriminant when considering referral for cardiac surgery on both clinical and economic grounds. This is especially true for acute disorders, as elderly patients are said not to tolerate such surgery as well as younger patients. Rupture of the interventricular septum is a disastrous complication of acute myocardial infarction and carries a high mortality despite surgical intervention. We reviewed the experience of this hospital with elderly patients with this condition and of the referral pattern from peripheral hospitals. From 1980 to 1989,108 patients with postinfarction septal rupture were seen. Twenty two patients were $\geqslant 70$ years of age and 86 were $<70$. Site of infarction before rupture was anterior in $68 \%$ of those $\geqslant 70$ and $63 \%$ in those $<70$. The spectrum of cardiac decompensation after rupture was similar in the two age groups although heart rate was lower (101 v $110 / \mathrm{min}, \mathrm{p}=0.037$ ) and systolic blood pressure higher $(100 v 92 \mathrm{~mm} \mathrm{Hg}, \mathrm{p}=0.1)$ in patients $\geqslant 70$. Extent of coronary artery disease was similar ( $2 v 1.88$ vessel disease). Intention to treat at our coronary care unit was for urgent repair in $6(27 \%) \geqslant 70,(56(65 \%)<70)$; medical management before proposed repair in $12(55 \%) \geqslant 70,(22(26 \%)$ $<70$ ); and four $(18 \%) \geqslant 70$, (eight $(9 \%)<70$ ) were considered unsuitable for surgery $(\mathrm{p}<0.01)$. Thirteen $(59 \%)$ $\geqslant 70$ had surgical repair of whom $10(77 \%)$ survived; 68 $(79 \%)<70$ had surgery of whom $33(49 \%)$ survived. Of the admissions from the local population (79000), 13/34 $(38 \%)$ were $\geqslant 70$; only $9 / 74(12 \%)$ patients referred from peripheral hospitals (population 809000 ) were $\geqslant 70$ years of age $(p<0.01)$. 
Age alone should not deter referral for assessment as careful selection can produce an acceptable surgical mortality that compares favourably with that in a younger population in whom more elaborate surgery is often attempted. Referral rates from peripheral hospitals are low at all ages compared with the frequency of rupture found in the local population but referral of those $\geqslant \mathbf{7 0}$ is disproportionately low.

\section{Automatic recording of defibrillation on general medical wards by day and night: implications for ward design and staffing}

\section{Wright, J Barrister, A F Mackintosh \\ St James's University Hospital, Leeds}

The outcome of ventricular fibrillation on general medical wards could depend upon the speed of defibrillation, particularly at night. Defibrillation was timed accurately on five of the six general medical wards. Each ward had a cardiac arrest call button, and the time of all calls was logged automatically by the hospital switchboard. Most patients were located in four-bed bays within $50 \mathrm{~m}$ of a defibrillator with a Tracker recorder to record automatically the time of switch on and first defibrillation, and all monitored rhythms. In 26 months, 67 recordings were obtained from 69 first episodes of ventricular fibrillation. Twenty eight patients were initially resuscitated, and 17 survived to be discharged. Major non-cardiac illness before ventricular fibrillation was associated with increased mortality $(4 / 41 v 13 / 27,1$ set of data incomplete, $p=0.001)$. Ventricular fibrillation that occurred during the daytime nursing shift (07:30-15:30 hours) was associated with increased survival compared with ventricular fibrillation at other times $(10 / 23 v 7 / 46$, $\mathrm{p}=0.01$ ). No patient who developed ventricular fibrillation between 20:00 hours and 04:00 hours survived. Ventricular fibrillation was evenly distributed over the 24 hour period and, importantly, there was no significant diurnal variation in response intervals. Outcome was not related to the time from cardiac arrest call to monitor connection (median $125 \mathrm{~s}$, range 0-620), or the time from call to defibrillation (median $181 \mathrm{~s}$, range 37-662). Ventricular fibrillation was displayed for longer than $60 \mathrm{~s}$ before the first defibrillatory shock in 27 recordings, and four patients were given further shocks without clear indication.

This automatic recording system provides an effective audit of defibrillation. The poor results at night could be due to late detection of cardiac arrests, or less efficient initial resuscitation. Staffing levels were reduced at night, and night shift staff may have difficulty attending resuscitation training. Survival might be better on open plan wards.

\section{Coronary vasomotor effects of serotonin in patients with angina: relation to coronary stenosis morphology}

D Tousoulis, E McFadden, J Clarke, J C Kaski, A Maseri, G Davies

Cardiology Department, Royal Postgraduate Medical School, Hammersmith Hospital, London

Previous experimental studies have shown that the effect of serotonin (5-HT) on a coronary stenosis depends on whether that stenosis is compliant or fixed. The relation, however, between morphology of coronary stenosis and the response to 5-HT in patients with angina is not known. Using computerised quantitative coronary angiography we studied the effects of intracoronary infusion of 5-HT on 38 coronary stenoses of different morphology (concentric, eccentric, complicated) in 11 patients with stable angina and four with variant angina. In response to the maximum infused concentration of $5-\mathrm{HT}, 100 \%$ of complicated stenoses and $50 \%$ of concentric stenoses constricted by $>20 \% \quad(p<0.05)$. The magnitude of constriction was greater at eccentric stenoses $(32 \cdot 08(4 \cdot 1) \%)$ than concentric stenoses $(15.68(2.8) \%, p<0.05)$, and greater at complicated stenoses $(57.69 \quad(7.6) \%, p<0.05)$ than eccentric stenoses. At complicated stenoses the constriction was greater $(0.85(0.16) \mathrm{mm}, \mathrm{p}<0.05)$ than at the adjacent reference segments $(0.42(0.12) \mathrm{mm})$. It was similar to the reference segment for both concentric and eccentric stenoses. The constriction at the stenosis was greater for complicated lesions than for smooth (concentric and eccentric) lesions in both patients with stable (51.8 $(7.3) v 22.5(4.1) \%, \mathrm{p}<0.001)$ and variant $(77(17) v 28.2$ $(8.1) \%, p<0.05)$ angina. There was a weak correlation $(r=0.38)$ of magnitude of constriction with length of stenosis, but not with severity of baseline stenosis.

In these patients the magnitude of the vasoconstrictor response to 5-HT at the site of an atheromatous coronary plaque depends on the morphological characteristics of the plaque and is more closely related to irregular contour than severity or length of stenosis. This relation suggests that variations in receptor type, or density, or in the smooth muscle cell response to stimulation may determine the response to locally released 5-HT in patients with coronary disease.

\section{Invere relation between serum lipoprotein (a) concentrations and first phase insulin secretion}

M Sidhu, D Crook, I Godsland, C Walton, V Wynn, M Oliver

Wynn Institute for Metabolic Research, London

Elevated serum lipoprotein (a) $(\mathrm{Lp}(\mathrm{a}))$ concentrations are often seen in patients at high risk of coronary heart disease. Recently, raised LP(a) concentrations have been reported in both insulin dependent and non-insulin dependent diabetes mellitus, conditions associated with increased risk of coronary heart disease. Consequently, it has been suggested that raised serum concentrations of $L p(a)$ contribute to the increased risk of coronary heart disease in these subjects. To investigate whether an association exists between serum $\mathrm{Lp}(\mathrm{a})$ concentrations and insulin metabolism, serum $\mathrm{Lp}(\mathrm{a})$ was measured in 146 healthy non-obese men in whom insulin metabolism had been assessed by an intravenous glucose tolerance test with measurement of plasma concentrations of glucose, insulin, and C-peptide. When subjects were stratified according to quartile of serum $\mathrm{Lp}(\mathrm{a})$ distribution, an inverse relation was seen with the first phase insulin response to glucose. This finding was supported by further analysis of these data using mathematical modelling, which showed an inverse relation between serum $\mathrm{Lp}(\mathrm{a})$ and both first phase insulin secretion and responsiveness. No association was seen between serum $L p(a)$ concentration and insulin resistance, implying that $\mathrm{Lp}(\mathrm{a})$ operates independently of the constellation of metabolic disturbances associated with coronary heart disease (Reaven's "Syndrome X"), which centre on the state of insulin resistance. 
These findings suggest a role for $L p(a)$ in the development of chronic atherosclerotic complications in diabetic patients as well as increasing risk of coronary heart disease in healthy subjects.

\section{Mechanisms of intracellular pH recovery after global ischaemia in the perfused heart}

\section{J I Vandenberg, J C Metcalfe, A A Grace \\ Department of Biochemistry, University of Cambridge}

The mechanisms of recovery of intracellular $\mathrm{pH}\left(\mathrm{pH}_{\mathrm{i}}\right)$ on reperfusion after ischaemia are not well understood. We have examined the relative roles of: (a) the $\mathrm{Na}^{+}-\mathrm{H}^{+}$ antiport; (b) $\mathrm{HCO}_{3}{ }^{-}$dependent acid extrusion; and (c) lactate efflux, on the recovery of $\mathrm{pH}_{\mathrm{i}}$ after total global ischaemia in the Langendorff perfused ferret heart. In hearts perfused with either $\mathrm{HCO}_{3}{ }^{-}$or nominally $\mathrm{HCO}_{3}$ free HEPES buffer the $\mathrm{Na}^{+}-\mathrm{H}^{+}$antiport was inhibited by $1 \mu \mathrm{M}$ ethylisopropylamiloride $\left(\mathrm{K}_{\mathrm{i}}=0 \cdot 15 \mu \mathrm{M}\right)$. Total global ischaemia was produced by clamping the inflow line for 10 min. $\mathrm{pH}_{\mathrm{i}}$ was estimated using ${ }^{31} \mathrm{P}$-nuclear magnetic resonance spectroscopy and effluent was collected for $2.5 \mathrm{~min}$ after reperfusion for lactate assay. Results are expressed as mean (SD). In $\mathrm{HCO}_{3}{ }^{-}$-solution $\mathrm{pH}_{\mathrm{i}}$ fell from $7.08(0.02)(n=5)$ to $6.83(0.02)$ after $10 \mathrm{~min}$ ischaemia. On reperfusion $\mathrm{pH}_{\mathrm{i}}$ recovered at $0 \cdot 180(0.05) \mathrm{pH}$ units $/ \mathrm{min}$. In HEPES $\mathrm{pH}_{\mathrm{i}}$ fell from $7.05(0.03)(\mathrm{n}=5)$ to $6.78(0.02)$ after $10 \mathrm{~min}$ ischaemia and recovered at $0.116(0.02) \mathrm{pH}$ units/min on reperfusion ( $\mathrm{p}<0.05 v \mathrm{HCO}_{3}{ }^{-}$). In HEPES with the $\mathrm{Na}^{+}-\mathrm{H}^{+}$antiport blocked $\mathrm{pH}_{\mathrm{i}}$ fell from 7.00 $(0.04)(\mathrm{n}=4)$ to $6.77(0.03)$ after $10 \mathrm{~min}$ ischaemia and recovered at $0.073(0.04) \mathrm{pH}$ units $/ \mathrm{min}$ on reperfusion $\left(\mathrm{p}<0.05 v \mathrm{HCO}_{3}\right.$; NS $v$ HEPES). After $10 \mathrm{~min}$ ischaemia $6(1 \cdot 5) \mu \mathrm{mol}$ lactate $/ \mathrm{g}$ wet weight $(\mathrm{n}=15)$ effluxed from the heart in the first $2.5 \mathrm{~min}$ of reperfusion. Proton coupled lactate efflux under the conditions of these experiments can account for $50 \%$ of $\mathrm{pH}_{\mathrm{i}}$ recovery in $\mathrm{HCO}_{3}{ }^{-}$perfused hearts, $80 \%$ in nominally $\mathrm{HCO}_{3}{ }^{-}$free buffer, and $100 \%$ in the nominal absence of $\mathrm{HCO}_{3}{ }^{-}$with the $\mathrm{Na}^{+}-\mathrm{H}^{+}$antiport blocked (assuming an intracellular proton buffering capacity of approximately $25 \mu \mathrm{mol} / \mathrm{g}$ wet weight).

The $\mathrm{Na}^{+}-\mathrm{H}^{+}$antiport is not the major mediator of the recovery of $\mathrm{pH}_{\mathrm{i}}$ after global ischaemia in the perfused ferret heart. Recovery of $\mathrm{pH}_{\mathrm{i}}$ is principally a result of activity of an eternal $\mathrm{HCO}_{3}{ }^{-}$dependent mechanism and a second process consistent with proton coupled lactate efflux.

\section{Closure of arteriovenous fistulas in renal transplant recipients: long-term haemodynamic benefits}

\section{J C Doig, A Heads, T J H Goodship, R Wilkinson, $S$ Hunter \\ University Department of Cardiology, Freeman Hospital, Newcastle upon Tyne}

The longer-term haemodynamic consequences of planned surgical closure of arteriovenous fistulas in patients after successful renal transplantation have not been described. This study measured exercise performance on treadmill, echocardiographic estimation of chamber dimensions and Doppler cardiac output in a group of 11 patients (mean age 41) with normal renal function at a mean of 21 months after renal transplantation. They were studied before closure and at a mean of five months after closure. Five of these patients were reinvestigated at a mean of 15 months after closure. Exercise duration increased by $10 \%$ by five months with respect to initial values $(621$ (206) to 684 (192), $p=0.004)$. Systolic blood pressure and rate pressure product at peak exercise were not significantly different. Resting systolic blood pressure was unchanged. In those patients followed up for 15 months, there was a further $4 \%$ increase in exercise times $(455(161)$ to 537 (163) $\mathrm{s}, \mathrm{p}=0.021$, to $560(150) \mathrm{s}, \mathrm{p}=0.017$ ). There was no significant change in peak blood pressure or maximal ratepressure product. Stroke distance fell by $13 \%$ by five months $(28.2(5.1)$ to $24.6(4.8) \mathrm{cm}, \mathrm{p}=0.004)$ and remained lower at 15 months. Cardiac output fell by $10 \%$ at five months and remained lower at 15 months $(6 \cdot 1$ (1.6) to $5.5(1.5)$ to $4.8(0.3) 1 / \mathrm{min}, \mathrm{p}=0.024)$. Left ventricular end systolic and end diastolic dimensions were not significantly altered at either follow up time, but there was a trend to lesser left ventricular hypertrophy. Posterior wall thickness fell from $14.6(1.9)$ to $12.9(1.1) \mathrm{cm}$, and septal thickness reduced from $17.4(2.8)$ to $15.0(1.8) \mathrm{cm}$, $\mathrm{p}=0.039$. Medication was constant during the study and patients noticed a subjective improvement.

This study shows that short-term haemodynamic improvements are continued to the longer-term after elective surgical closure of arteriovenous fistulas in patients with normal renal function after renal transplantation. Patients have a lower resting cardiac output than before closure and are able to exercise for longer at the same work expenditure. These findings strengthen the case for routine closures of fistula after successful renal transplantation.

\section{Prolonged QT interval in type 1 diabetes: information from idential twins}

\section{S S S Lo, R D G Leslie, M G St John Sutton}

Diabetic Unit, Westminster Hospital; and Royal

Brompton National Heart and Lung Hospital, London

Type 1 diabetic people suffer sudden death from cardiorespiratory arrest. To determine whether QT interval prolongation may provide a substrate for this sudden death and whether this is related to autonomic neuropathy, we assessed QT interval in 34 monozygotic twin pairs discordant for type 1 diabetes and compared with 34 normal control subjects of similar age and sex. The twins (16 male) were aged from 10 to 42 years and the diabetic twins had a mean disease duration of 15 years. All subjects were normotensive, had no clinical cardiac ischaemia, and were taking no medications except for insulin in the diabetic twins. Autonomic neuropathy was assessed as $(a)$ the fall in systolic blood pressure after one min of standing ( $>20 \mathrm{~mm} \mathrm{Hg}=$ abnormal); and $(b)$ the changes in heart rate during deep breathing, during Valsalva manoeuvre, and on standing. These tests were assessed by computer and graded by a conventional protocol. Resting heart rate, corrected QT interval, and the frequency of autonomic neuropathy were all significantly higher in diabetic twins than their non-diabetic co-twins (81 (13) $v 72$ (15) beats/min, $\mathrm{p}<0.0005 ; 429$ (18) $v 408$ (13) ms, p < 0.005; and $24 \% v 3 \%, \mathrm{p}<0.02$ respectively) but there were no differences between the non-diabetic twins and normal control subjects. Twins with diabetes $>15$ years $(n=17)$ had a significantly longer corrected $Q T$ interval than their non-diabetic co-twins (423 (18) $v 408$ (12) ms, p < 0.005); by contrast, this difference was not detected in twins with shorter duration of disease. Diabetic 
twins with autonomic neuropathy had a similar corrected QT interval $(n=8)$ when compared with diabetic twins without autonomic neuropathy $(n=26)$. Prolongation $(>440 \mathrm{~ms}$ ) of corrected QT interval was present in $4 / 34$ $(12 \%)$ diabetic twins, two of whom did not have autonomic neuropathy. In long standing type 1 diabetes, corrected QT interval may be prolonged, is not related to autonomic neuropathy, and may provide the substrate for sudden death.

\section{A novel monoclonal conjugate with powerful antiplatelet and fibrinolytic properties which localises to damaged endothelium}

\section{R S More, M Brack, S Pringle, A H Gershlick Glenfield General Hospital, Leicester}

We have developed a molecular conjugate (R2) comprising an anti-platelet glycoprotein, a IIb/IIIa (M735) monoclonal antibody (MAb), an antidamaged endothelial cell MAb (P14G11), and urokinase (Ur) using succinimidyl 3-(2-pyridyldithio) propionate (SPDP) as a coupling reagent. Specific Ur activity of R2 was 10000 $\mathrm{IU} / \mathrm{mg}$ protein. Fibrinolytic activity of the conjugate was confirmed with fibrin plates and a chromogenic substrate assay for Ur. Whole platelet preparations confirmed targeting of $R 2$ for platelets. Localisation of $R 2$ to damaged endothelial cells was shown using scratch damaged endothelial monolayers and peroxidase coupled second antibody technique. Inhibition of platelet aggregation was assessed with $20 \mu \mathrm{M}$ adenosine diphosphate and $4 \mu \mathrm{g} / \mathrm{ml}$ collagen as agonists and five $\mu \mathrm{g}$ of $\mathrm{R} 2$ completely inhibited platelet aggregation (Ag) induced by either of these reagents. By contrast $12 \mu \mathrm{g}$ of $\mathrm{M} 735$ alone was required to inhibit platelet aggregation by collagen and this dose only decreased platelet aggregation with adenosine diphosphate to $66 \%$ of control. The effect of $\mathrm{R} 2$ on coagulation was shown to be at least equivalent to Ur and M735 alone (whole blood clotting ratio for $\mathrm{R} 2=1 \cdot 26, \mathrm{Ur}=1 \cdot 13$, M735 = 1.18).

A conjugate with enhanced anti-platelet and thrombolytic properties has been developed that localises to sites of damaged endothelium such as occur after coronary angioplasty. Such molecules will be tested for their effects on acute closure and medium term restenosis.

\section{Real time, echocardiographic analysis of ventricular function by automatic boundary detection}

\section{J Monaghan, M R Thomas, L K Michalis,} D E Jewitt

Department of Cardiology, King's College Hospital, London

Left and right ventricular function may be studied in real time by new methods of echo signal processing based upon tissue characterisation. Identification and separation of blood and myocardial tissue structures is performed automatically down each image scan line. From this, the blood tissue interface is recognised over the entire image and used to create an endocardial boundary. An operator defined region of interest may be drawn over the left or right ventricles and the ventricular area is continuously calculated for each image frame. A real time graphic display of ventricular area is displayed together with the fractional area change (FAC), an ejection fraction analogue, which is updated for each cardiac cycle. Assessment of ventricular contractility or filling rates is obtained by real time analysis of rate of area change (dA/dT). This system has been currently evaluated in 20 unselected patients undergoing routine echocardiographic study. A total of 160 parasternal short axis or apical four chamber imaging planes were evaluated for ventricular function analysis. Two operators scanned each patient to allow analysis of inter and intraobserver variability. 51 $(31 \%)$ image studies were rejected because successful tracking of at least $75 \%$ of the endocardial boundary could not be obtained. In those studies where good endocardial tracking was obtained, inter and intraobserver variability respectively for ventricular area $(15 \%, 12 \%)$, FAC $(16 \%$, $11 \%)$, and $\mathrm{dA} / \mathrm{dT}(13 \%, 11 \%)$ were obtained. The average study time was $4 \cdot 25(1 \cdot 1)$ minutes.

Thus this new system provides rapid real time, noninvasive assessment of ventricular function and it may be used successfully in most patients with acceptable variability. It is clearly of potential value in the study of the effect of therapeutic interventions and therefore warrants further validation and clinical assessment in this context.

\section{High apolipoprotein(a) concentrations are associated with increased cardiac risk in patients undergoing peripheral vascular surgery}

P H Groves, N P Lewis, R J Whiston, E A Jones, W D Evans, A Rees, M Ruttley, I F Lane, R J C Hall Departments of Cardiology, Medicine, Radiology and Surgery, University Hospital of Wales, Cardiff

Apolipoprotein(a) (apo(a)) concentrations are genetically determined and are associated with the presence of coronary artery disease in patients with peripheral vascular disease. The close structural homology that exists between apo(a) and plasminogen has implied an important link between lipoprotein metabolism and the clotting/ fibrinolytic system. We have investigated the role of apo(a) analysis in the preoperative cardiac risk assessment of 39 consecutive patients undergoing peripheral vascular surgery. Apo(a) concentration was measured in fasting serum by an immunoradiometric assay with monoclonal antibodies to two different epitopes of apo(a). Coronary arteriography and dipyridamole thallium scintigraphy were also performed in all subjects. Perioperative cardiac complications occurred in six patients $(15 \%$; one of which was fatal). Of these six patients, five had apo(a) concentrations that were greater than the cohort median (197 U/1; sensitivity $v$ specificity $83 \% v 52 \%$ ), five had appreciable coronary artery disease ( $\geqslant$ one vessel $\geqslant 50 \%$ diameter stenosis; $83 \%$ v 36\%), and four had one or more reversible thallium defects $(67 \%, v 13 \%)$. Patients with apo(a) concentrations $>197 \mathrm{U} / 1$ had a 5.3-fold increase in the prevalence of cardiac complications. All three tests were abnormal in the patient who died.

These results indicate that high concentrations of apo(a) are associated with an increased incidence of perioperative cardiac complications in patients undergoing peripheral vascular surgery. The measurement of apo(a) concentrations may prove to be a simple and reliable means of identifying those at greatest risk and may emerge as a useful additional tool in the preoperative assessment of this group of patients. 
Ventricular interactions in pulmonary hypertension: impaired left ventricular filling caused by prolonged right ventricular systole

S J D Brecker, B B Stojnic, H B Xiao, D G Gibson Cardiac Department, The Royal Brompton National Heart and Lung Hospital, London

To examine the effects of pulmonary hypertension ( $\mathrm{PH})$ upon left ventricular diastolic events, we studied 29 patients with $\mathrm{PH}$ and 10 controls with an enlarged right ventricle but normal pulmonary artery pressure, with echocardiography and Doppler and phonocardiography. Septal thickness, cavity dimensions, right ventricularatrial (RV-RA) gradient, isovolumic relaxation time, and left ventricular filling patterns were measured. The peak RV-RA gradient was 60 (16) $\mathrm{mm} \mathrm{Hg}$ in PH patients, and 18 (5) $\mathrm{mm} \mathrm{Hg}$ in controls. The time intervals of pulmonary closure to the end of the tricuspid regurgitation and to the onset of tricuspid flow were both prolonged in patients with PH compared with controls $(115$ (60) $\mathrm{ms}$ and 120 (40) $v 40(15)$ and $45(10)$ (mean (SD)), $\mathrm{p}$ values $<0.001$ ). On transmitral Doppler, 23 of 29 patients with $\mathrm{pH}$ had a dominant A wave; all controls had a dominant $E$ wave. Left ventricular isovolumic relaxation time was prolonged in PH compared with controls, both as aortic closure to mitral valve opening (80 (25) $\mathrm{ms} v 50(15))$, and to the onset of mitral flow $(105(30) \mathrm{ms} v 60(15)$, p values $<0.001)$. The delay from mitral opening to the onset of transmitral flow was longer in PH patients compared with controls (30 (15) ms $v 10(10), p<0.001)$. At the time of mitral opening, there was a RV-RA gradient of $12(10) \mathrm{mm} \mathrm{Hg}$ in $\mathrm{PH}$, and on $\mathrm{M}$-mode, 20/29 patients had evidence of posterior septal displacement during isovolumic relaxation. The RV-RA gradient was negligible in controls $(0.4(0.3), \mathrm{p}<0.001)$, and none had septal displacement $(0 / 10 v 20 / 29$, $\mathrm{p}<0.0001$ ).

Prolonged right ventricular tension decline, the direct result of severe $\mathrm{PH}$, causes prolonged tricuspid regurgitation. It persists until after mitral opening on the left side of the heart, where events during isovolumic relaxation are disorganised, and subsequent filling impaired. These effects are likely to be mediated through the interventricular septum; this right and left ventricular asynchrony may represent an unrecognised mode of ventricular interaction.

\section{Balloon dilatation of valves in infants and children with carbon dioxide as the contrast agent}

\author{
N Sreeram, K Walsh, I Peart, R Arnold \\ Heart Clinic, Royal Liverpool Children's Hospital, \\ Liverpool
}

We used carbon dioxide gas $\left(\mathrm{CO}_{2}\right)$ as the contrast agent for balloon dilatation of valves in 47 infants and children (25 male, 22 female). In addition to the peak systolic withdrawal gradient at catheterisation, the peak instantaneous gradient was also derived using continuous wave Doppler ultrasound 24 hours before, and 24 hours after the procedure. Balloon dilatation of the aortic valve was performed in 20 patients (including four neonates and four patients who had previously undergone surgical valvo- tomy) at a median age of 43 months (range 0.07-264) with a balloon to annulus ratio (mean (SEM) of $1 \cdot 15(0 \cdot 05)$ ). The peak systolic withdrawal gradient fell from 59 (5) to 22 (3) $\mathrm{mm} \mathrm{Hg} \quad(p<0.01)$. New or increased aortic regurgitation occurred in 13 patients, but was < grade 3 angiographically in all but two cases. The peak instantaneous gradient fell from 78 (5) to 34 (3) $\mathrm{mm} \mathrm{Hg}$ $(\mathrm{p}<0.01)$, and has remained virtually unchanged (40 (6) $\mathrm{mm} \mathrm{Hg}$ ) over a follow up period of 5 (1) months. In 14 patients with pulmonary stenosis (median age 18 months; range 5-129) and with a balloon to annulus ratio of 1.46 $(0.07)$ balloon dilatation reduced the peak systolic withdrawal gradient from 54 (9) to 20 (4) $\mathrm{mm} \mathrm{Hg}$ $(\mathrm{p}<0.01)$, and the peak instantaneous gradient from $67(7)$ to 27 (5) $\mathrm{mm} \mathrm{Hg}(\mathrm{p}<0.01)$. In one patient with predominant infundibular stenosis there was no change in the gradient. At follow up (mean 5 (1) months) the mean peak instantaneous gradient was $23(6) \mathrm{mm} \mathrm{Hg}$. In 11 patients with tetralogy of Fallot (median age 3 months; mean balloon to annulus ratio $1 \cdot 8(0.03)$ ) balloon dilatation of the pulmonary valve produced an immediate increase in systemic arterial oxygen saturation from $75 \%$ (16) to $87 \%$ (12) $(p<0.01)$. Over a follow up period of $6 \cdot 6(1 \cdot 2)$ months two patients have required further palliation with an aortopulmonary shunt.

$\mathrm{CO}_{2}$ allows for rapid balloon inflation and deflation at low inflation pressures, with minimal disturbance of systemic blood pressure and cardiac rhythm. The greatly reduced inflation and deflation times enable the use of larger diameter balloons on smaller shafts, with less vascular trauma.

Effect of intravenous magnesium on tachycardia and cardiac arrest in acute myocardial infarction

\section{S M Horner \\ Middlesex Hospital, London}

The use of intravenous magnesium in acute myocardial infarction is currently undergoing investigation in those treated with thrombolysis. The trials predating thrombolysis, however, are important as they allow the effect of magnesium alone to be quantified, against which its effect with thrombolysis can be judged, and they also provide the only information available on the effects of magnesium in those in whom there is a contraindication to thrombolysis. Nine randomised controlled trials concerning 984 patients with acute myocardial infarction were analysed. Eight of these were double blind and one was single blind. Intravenous magnesium administration was associated with a $49 \%$ reduction in ventricular fibrillation and tachycardia ( $p=0.0004,95 \%$ CI $24-66 \%$ ). Five trials (467 patients) also noted the incidence of other forms of cardiac arrest and supraventricular tachycardia. There was a $72 \%$ (8 to $93 \%$ ) reduction in the incidence of electromechanical dissociation $(p=0.02$, and a $60 \%$ $(-86 \%$ to $11 \%)$ reduction in the incidence of asystole $(p=0.04)$. The incidence of supraventricular tachycardias was reduced by $49 \%(0.3$ to 0.89$)(p=0.009)$, in the group treated with magnesium.

In the subset of patients not receiving thrombolysis for acute myocardial infarction intravenous magnesium would be expected to substantially reduce the incidence of tachyarrhythmias and cardiac arrest. 
Rapid exclusion of acute myocardial infarction in patients without SR elevation by serial enzyme analysis with novel analysers: myoglobin, creatine kinase, and creatine kinase-MB enzymes

H S Lee, S J Cross, P Garthwaite, K P Jennings Cardiac Department, Aberdeen Royal Infirmary; and Department of Mathematical Science, University of Aberdeen

Early exclusion of acute myocardial infarction within six hours of onset in patients without ST elevation is desirable and should have important impact on the management of coronary care units, accident and emergency units, and the appropriate use of thrombolysis. This study set out to determine: (a) If myoglobin, creatine kinase, and creatine kinase-MB can be used for the early diagnosis of myocardial infarction (within six hours) in patients without ST elevation. (b) The optimal timing of blood collection for enzyme analysis. (c) If rapid analysers are accurate enough to provide the diagnosis. Fifty nine consecutive patients with suspected myocardial infarction were recruited in a prospective study. They consisted of 13 patients with myocardial infarction (seven without ST elevation) and 48 patients in whom infarction was subsequently excluded. Enzyme assays were performed by novel quantitative rapid analysers (result within 10 minutes) situated in the coronary care unit. Optimal timing for blood collection was determined by hourly sampling for six hours. A myoglobin threshold of $120 \mu \mathrm{g} / 1$ at four hours after the onset of chest pain gave a sensitivity of $88.56 \%$ for diagnosing myocardial infarction in patients without ST elevation, a specificity of $99.80 \%$, and predictive accuracy of $96.80 \%$. At six hours this was $97.92 \%, 99.73 \%$, and $98.65 \%$ respectively. No patient with myoglobin $<120$ $\mu \mathrm{g} / 1$ at four hours was found subsequently to have infarcted. For creatine kinase-MB a threshold of $20 \mathrm{U} / 1$ at six hours gave corresponding figures of $81.55 \%, 92.25 \%$, and $93 \cdot 18 \%$

Myocardial infarction can be excluded within four hours by myoglobin analysis or six hours by creatine kinase-MB analysis. This permits patients at low risk of events to be moved early from the coronary care unit freeing beds for other patients.

\section{Recovery of coronary resistive vessel function after successful coronary angioplasty}

N G Uren, T Crake, D C Lefroy, R de Silva, G J Davies, A Maseri

Hammersmith Hospital, London

Eleven male patients (mean age 54, range 44-67) with single vessel, left anterior descending (LAD) coronary artery disease, positive exercise electrocardiograms, and normal left ventricular function were studied before and after successful percutaneous transluminal coronary angioplasty (PTCA). The coronary vasodilator response (CVR = maximal $/$ basal myocardial blood flow (MBF) to intravenous dipyridamole $(0.5 \mathrm{mg} / \mathrm{kg}$ over four min) was determined from Doppler measurement of coronary flow velocity and quantitative angiography. There was no significant change in CVR immediately after PTCA (mean (SD) $1.62(0.44)$ before PTCA v $1.66(0.60)$ after PTCA).
In nine patients (eight with negative exercise electrocardiograms at seven days), basal and maximal regional $\mathrm{MBF}$, and CVR, were determined at one, seven and 100 days after PTCA using dynamic 15 slice positron emission tomography with $\mathrm{H}_{2}{ }^{15} \mathrm{O}$. Regional $\mathrm{MBF}$ measurements from myocardial regions subtended by the LAD (PTCA region) were compared with those from a myocardial region subtended by an angiographically normal artery (remote region). At one, seven, and 100 days after PTCA in eight patients without restenosis, basal MBF was 1.25 $(0.26), 1 \cdot 11(0 \cdot 16)$, and $1.07(0.07) \mathrm{ml} / \mathrm{min} / \mathrm{g}$ respectively in the PTCA region, and $0.93(0.16)(\mathrm{p}<0.01), 0.87(0.15)$ $(\mathrm{p}<0.05)$, and $0.96(0.11) \mathrm{ml} / \mathrm{min} / \mathrm{g}$ in the remote region. At one, seven, and 100 days after PTCA, MBF after dipyridamole was $2.13(0.64), 1.97(0.61)$, and $2.29(1.00)$ $\mathrm{ml} / \mathrm{min} / \mathrm{g}$ in the PTCA region, and $2.73(0.90)(\mathrm{p}<0.05)$ $2.46(1.07)$, and $2.32(0.57) \mathrm{ml} / \mathrm{min} / \mathrm{g}$ in the remote region, respectively. Thus the calculated CVR was $1.76(0.60)$, $1.76(0.48)$, and $2.15(0.97)$ in the PTCA region and 2.99 $(1.03)(p<0.01), 2.82(1.07)(p<0.05)$, and $2.42(0.56)$ in the remote region at one, seven and 100 days respectively.

Hence, in these patients, the CVR in the PTCA region is impaired immediately after successful PTCA and this impairment persists for up to one week. This impaired CVR within the first 24 hours is due both to an increase in basal MBF and a decrease in maximal resistive vessel dilatation, and at seven days it is due to a persistent increase in basal MBF. By 100 days, in the absence of restenosis, resistive vessel function has returned to normal.

\section{Independent value of heart rate variability for predicting sudden death after myocardial infarction}

O Odemuyiwa, M Malik, T Farrell, A Staunton, J Poloniecki, T Millane, A J Camm

Department of Cardiological Sciences, St George's Hospital Medical School, London

The independent prognostic value of reduced heart rate variability for sudden death after myocardial infarction is still controversial. Therefore, predischarge clinical assessments and left ventricular angiography were carried out in 411 survivors of a first myocardial infarction who were followed up for between one month and five years. Heart rate variability was derived from predischarge Holter recordings as the baseline width of the frequency histograms of normal to normal RR intervals. Of the 411 patients, $226(55 \%)$ received thrombolytic therapy and 173 $(42 \%)$ were discharged on $\beta$ blockers. There were 103 $(25 \%)$ patients with left ventricular ejection fractions $<40 \%: 82(20 \%)$ of the patients were treated for heart failure in hospital. The predictive value of reduced heart rate variability $(<150 \mathrm{~ms}$ ) for sudden death was examined using proportional hazards analysis. There were $13(3 \%)$ sudden deaths. The estimated probability of sudden death in the first year was $4 \%$ without heart rate variability in the model. Given heart rate variability, the estimated probability of sudden death in the first year was 9\% if heart rate variability was $<150 \mathrm{~ms}$, but only $1 \%$ if $>150 \mathrm{~ms}$ (relative risk $8 \cdot 3 ; 95 \% \mathrm{CI}: 2 \cdot 2-30 \cdot 7$; proportional hazards regression coefficient $-2 \cdot 12$ ).

Reduced heart rate variability provides independent prognostic information in survivors of acute myocardial infarction. 
Giving up smoking: how rapidly does risk of heart attack disappear?

Jin-ling Tang, D G Cook, A G Shaper

Royal Free Hospital School of Medicine, London

There is disagreement about the time required for the smoking associated risk of ischaemic heart disease to disappear after stopping smoking. The 9.5 year data from the British Regional Heart Study, a prospective study of cardiovascular disease among 7735 men age 40 to 59 years randomly drawn from one general practice group in each of 24 British towns, were examined to determine how rapidly the excess risk of ischaemic heart disease disappears after stopping smoking. The risk of ischaemic heart disease relative to never smokers was $2 \cdot 1(95 \%$ CI $0 \cdot 8-1 \cdot 6)$ in current cigarette smokers and ex-smokers respectively, after excluding men with a doctor's diagnosis of angina or previous heart attack and adjusting for age, systolic blood pressure, and serum total cholesterol concentration. Primary pipe or cigar smokers appear to carry no excess risk (relative risk $=1 \cdot 1(0 \cdot 5-2 \cdot 4)$ whereas secondary pipe or cigar smokers showed a relative risk of $1.8(2 \cdot 7-1 \cdot 2)$ which is nearly as high as in current cigarette smokers. Separating ex-smokers according to the number of years since stopping smoking before the initial screening, we found a significant trend of declining risk with increasing years since smoking. Thus, ex-smokers had some benefit in the first two and 3 to 5 years since smoking and most of the excess risk disappeared in 6 to 10 years. In light ex-smokers ( $<20$ cigarettes/day) no excess risk was seen after five years since smoking, but in heavy ex-smokers $(\geqslant 20$ cigarettes a day) the increased risk continued for up to 10 to 20 years after stopping. The benefit of stopping smoking was found to be more gradual than usually thought to be the case and switching from cigarettes to pipe or cigars does not appear to reduce the risk.

Although the benefit from stopping smoking should be stressed, emphasis should be put on educating the young not to start smoking and on advising current smokers to stop smoking altogether as early as possible.

\section{Altered coronary flow reserve and metabolism in myocardium subtended by normal arteries in patients with coronary artery disease}

N G Uren, P Marraccini, R Lorenzoni, D Nevola, $\mathbf{R}$ de Silva, $T$ Crake, $P$ G Camici

Medical Research Council Cyclotron Unit, Hammersmith Hospital, London

Myocardial blood flow (MBF) in regions subtended by angiographically normal arteries (ANA) was assessed in 13 patients (mean age 60,10 men) with single vessel disease by dynamic positron emission tomography with ${ }^{15} \mathrm{O}$-labelled water at rest and after intravenous dipyridamole $(0.56$ $\mathrm{mg} / \mathrm{kg}$ over four $\mathrm{min}$ ). Seven patients had left anterior descending disease (LAD) and six had right coronary artery or left circumflex disease (RCA/LCX). The coronary vasodilator reserve (CVR; dipyridamole $\mathrm{MBF}$ : resting $M B F$ ) in regions subtended by a stenosis was 1.87 $(0 \cdot 86)$, and in regions subtended by ANA it was $(2.90$ $(1.39))$. Both values were lower than the average CVR $(3.92$ $(0.89), \mathrm{p}<0.05$ for both comparisons) measured in 12 control men (mean age 30). Another group of 10 patients (mean age 60; nine men), five with LAD disease and five with RCA disease, and a control group of six patients (mean age 47; four men) underwent simultaneous arterial and great cardiac vein blood sampling during maximal atrial pacing to measure glucose extraction, alanine extraction, lactate extraction, and myocardial oxygen extraction in regions subtended by a diseased artery (LAD) or by an ANA, that is, the LAD in patients with RCA disease. Oxygen extraction was the same in the diseased region $(69(3 \%))$, the ANA region (67 (6)), and in controls (64 (7)). There was a decrease in lactate extraction in the LAD region $(-18(27) \%), p<0.05$ compared with the ANA region (38 $(17) \%)$, or controls $(26(11) \%)$. Glucose and alanine extraction were increased in both the diseased region, (8 (6)\% and $6(6) \%)$ respectively, and the ANA region (6 (3)\% and $4(3) \%)$, compared with controls (2 (3) $\%$ and $-1(3) \%$ ) ( $\mathrm{p}<0.05 v$ both diseased and ANA regions).

Thus CVR is reduced in regions subtended by ANA in patients with coronary artery disease elsewhere compared with control subjects; in addition, regions subtended by ANA are characterised by different glucose and alanine handling during pacing, although they do not show metabolic signs of ischaemia.

\section{Radiofrequency catheter ablation for atrioventricular accessory pathways}

\section{K Y Wong, V P Vassilikos, A W Nathan}

St Bartholomew's Hospital, London

Radiofrequency catheter ablation is an emerging treatment for arrhythmias due to atrioventricular accessory pathways (APs) such as those seen in the Wolff-Parkinson-White (WPW) syndrome. Forty seven patients, with 52 APs, had AP ablation performed over a one year period, and were followed up for one to 13 (mean six) months. Electrophysiological study was performed before ablation in 34 cases (two at other institutions); 13 had combined electrophysiological studies and ablation. Patients were aged 16 to 56 (mean 30), and 28 were men. Symptom duration was 0.2 to 40 (mean 11) years, and eight had suffered syncope. Patients had taken nought to six (mean 1.7) antiarrhythmic drugs, one had an anti-tachycardia pacemaker, and one had previous arrhythmia surgery. Thirty seven APs were overt (WPW) with a minimum pre-excited PR RR of 170 to 330 , mean $235 \mathrm{~ms}$, and 15 were concealed. Ebstein's anomaly was present in one, other conduction disease in one, and one had a large coronary sinus diverticulum. Patients received two to 43 (mean 12) $500 \mathrm{kHz}$ radiofrequency applications of up to $45 \mathrm{~W}$. Procedure time varied from 80 to 330 (mean 206) $\mathrm{min}$, and fluoroscopy time was eight to 125 (mean 57) min. Success in overt AP was $31 / 37(84 \%)$, but was only $6 / 15(40 \%)$ in concealed APs. Overall success was $37 / 52(71 \%)$ APs, and $33 / 47(70 \%)$ patients. Left free wall APs were successfully treated in $21 / 28,75 \%(17 / 20,85 \%$ in WPW), posterior septal in $9 / 13$, $69 \%(7 / 9,78 \%$ in WPW $)$, anterior septal in $6 / 8,75 \%(6 / 6$, $100 \%$ in WPW), and right free wall in $1 / 3,33 \%(1 / 2,50 \%$ in WPW). Reasons for failure included a coronary sinus diverticulum in one and complete failure to reach the appropriate part of the atrio-ventricular ring in four. Catheter manipulation was very difficult in several of the other failures-only one size, shape, and reach of deflectable tip catheter was available during this series. There was only one complication, a small pneumothorax after subclavian stab, which was treated medically. Three failures have been treated surgically, two await surgery, one has elected for medical treatment, and eight await another ablation. Patient success has improved from $66 \%$ in the first half of patients treated, to $74 \%$ in the second half.

Radiofrequency catheter ablation is an increasingly successful treatment for patients with APs. 


\section{Clinical experience with selective radiofrequency ablation in the treatment of atrioventricular nodal reentrant tachycardia}

\author{
S C Heald, Y Bashir, S O’Nunain, M Hamouda, \\ A J Camm, D E Ward \\ St George's Hospital, London
}

Transcatheter application of radiofrequency current is a technique with sufficient precision to allow selective ablation of slow or fast pathway conduction in patients with atrioventricular nodal reentrant tachycardia (AVNRT). We have attempted selective atrioventricular (AV) nodal modification in seven patients (aged 26-55; all women) with drug-refractory AVNRT. For fast pathway ablation, the catheter was positioned across the tricuspid valve to record the His potential then withdrawn slightly before the application of repeated radiofrequency pulses until persistent PR prolongation was obtained. In the slow pathway technique, mapping was performed along the septal leaflet of the tricuspid valve between the coronary sinus and His region and radiofrequency delivered at sites of possible pathway potentials. Six patients underwent AV nodal modification by the fast pathway approach, receiving 4 to 49 discharges. PR prolongation developed after 13/163 deliveries and transient high grade AV block was seen in 28/163. Mean $P R$ interval was prolonged by 79 (range 60-120) $\mathrm{ms}$ and AH interval by 50 (25-95) $\mathrm{ms}$. In four patients, ventriculoatrial conduction was abolished and AVNRT rendered non-inducible. In the other two, AVNRT was still inducible; in these patients, tachycardia persisted despite induction of high grade AV block with further radiofrequency pulses, presumably indicating interruption of $\mathrm{AV}$ nodal conduction below the tachycardia circuit; one required a permanent pacemaker. In two patients, slow pathway modification was attempted in the first instance. In one case, AV nodal duality was successfully abolished with no change in PR or AH interval and AVNRT was rendered non-inducible. In the other, this method was unsuccessful but fast pathway modification was subsequently effective. The five patients in whom AV nodal modification was successful by either approach have remained free of AVNRT during follow up 1 to 52 weeks.

Selective radiofrequency modification is an effective treatment for AVNRT. The fast pathway approach, however, may result in interruption of AV nodal conduction below the tachycardia circuit, allowing persistence of tachycardia despite PR prolongation.

\section{Catheter ablation for the cure of atrioventricular nodal reentrant tachycardia by destruction of the slow pathway}

\section{A W Nathan, V P Vassilikos, C K Y Wong \\ St Bartholomew's Hospital, London}

Atrioventricular nodal reentrant tachycardia (AVNRT) is one of the two common mechanisms for paroxysmal supraventricular tachycardia. Its circuit is thought to include a normal AV nodal ("fast") pathway, and an abnormal "slow" pathway. Selective ablation of the slow pathway should provide a physiological cure, whereas fast pathway ablation is associated with complete AV block in up to $10 \%$ of cases. We have treated six patients with recurrent AVNRT using radiofrequency ablation of the slow pathway. The patients were all women, aged 14 to 49 (mean 31 ), with symptom duration of nine to 38 (mean 17) years. Four had suffered syncope, and all had required multiple stays in hospital for their arrhythmias. They had been refractory to one to six (mean 3.7) anti arrhythmic drugs. One had an anti-tachycardia pacemaker. At electrophysiological study, AVNRT was induced in all, and dual anterograde pathway physiology was seen in all but one patient. Mapping was performed with a deflectable tipped, $2 \mathrm{~mm}$ spaced, electrode catheter. Slow pathway activity was thought to be evidenced by a discrete electrical potential, between the atrial and ventricular electrograms, always closer to the atrial electrogram, but preceding the His bundle electrogram detected on another catheter electrode. This site was on the inferior margin of Koch's triangle, superior and anterior to the ostium of the coronary sinus, 1 to $2.5 \mathrm{~cm}$ distant from the electrode recording the $\mathrm{His}$ bundle. At this site $500 \mathrm{kHz}$ radiofrequency energy of up to $45 \mathrm{~W}$ was delivered. The patients received four to nine (mean 6.2) bursts of energy. All procedures were performed with local anaesthesia, except in the 14 year old child. In the five patients with demonstrable AH jumps before the procedure, these were abolished in three, and were grossly exaggerated in the other two. At the end of the procedure, AVNRT could not be induced in any of the patients, despite the administration of atropine. Total procedure time, including electrophysiological study, was 135 to 270 (mean 182) minutes, with fluoroscopy times of 17 to 51 (mean 30) minutes. The only complication was a small pericardial effusion in one case, thought to be due to a right ventricular pacing electrode. All patients remain well and symptom free during follow up, except for one, who has had a single recurrence of AVNRT, and is awaiting a second procedure.

\section{Radiofrequency ablation of accessory} atrioventricular pathways: which electrogram characteristics are useful in the identification of successful target sites?

Y Bashir, S C Heald, S O'Nunain, M Hamouda, D Katritsis, A J Camm, D E Ward

St George's Hospital, London

Radiofrequency current represents a safe power source for ablation of accessory pathways (APs) but requires precise mapping for success. Radiofrequency ablation was attempted in 50 patients (age 11 to 66) with 51 APs (seven concealed) to control refractory or life threatening arrhythmias. Locations of APs were left free wall (26), posterior or mid septal (20); anteroseptal (one) and right free wall (four). Conduction was successfully abolished in 46/51 pathways $(90 \%)$ and has recurred in $3 / 46(7 \%)$ during follow up of 6 (5) months. Median duration of procedure (including $30 \mathrm{~min}$ for observation) was 140 (range 35-360) min and screening time was 44 (4-187) $\mathrm{min}$. The number of radiofrequency pulses varied from one to 51 (median 12) per session. Complications included pericarditis, (three cases) and complete heart block (one case). The optimum mapping criteria for recognition of appropriate target sites have not been established. Accordingly, we analysed the local bipolar electrograms from 406 consecutive sites of radiofrequency delivery in 30 patients with pre-excitation, all recorded via the ablating catheter $(4 \mathrm{~mm}$ tip, $2.5 \mathrm{~mm}$ interelectrode spacing) during anterograde mapping. Local atrioventricular time $(\mathrm{A}-\mathrm{V})$, time from delta wave onset to local ventricular activation ( $Q R S-V$ ), and the presence of a possible AP potential were noted. Comparing the 28 successful sites with all 378 unsuccessful sites, $A-V$ time was similar (41 (15) v 46 (14) ms; NS), QRS-V was more negative ( -3 (11) v 10 (15) $\mathrm{ms} ; \mathrm{p}<0.0001$ ), and AP 
potentials were commoner $(8 / 28 v 27 / 378 ; \mathrm{p}<0.001)$, but compared with 38 sites at which transient AP block developed, there were no significant differences in electrogram characteristics. A QRS-V $\leqslant 10$ ms identified successful sites with a sensitivity of $96 \%$ but positive predictive accuracy (PPA) was only 9.9\%. A QRS-V $\leqslant 0$ ms identified only $75 \%$ of successful sites (PPA $12 \cdot 1 \%$ ). Combination of QRS-V $\leqslant 10$ ms plus AP potentials was even less sensitive (29\%) and only slightly more specific (PPA 23\%).

Radiofrequency ablation of APs is safe and effective, but the poor specificity of electrogram characteristics for identifying appropriate target sites contributes to the lengthy procedure times required in some cases.

\section{A novel technique using a bipolar right ventricular/left ventricular electrode configuration for radiofrequency ablation of "difficult" septal pathways}

\author{
Y Bashir, S C Heald, D Katritsis, S O’Nunain, \\ A J Camm, D E Ward \\ St George's Hospital, London
}

Although the overall success rate for radiofrequency ablation of accessory pathways is high, particular difficulty may be encountered with accessory pathways located in the anatomically complex posterior septal region, including intermediate septal accessory pathways, some of which require lengthy procedures with increased radiation exposure and yet still result in failure. We describe a new technique for ablation of these "refractory" accessory pathways by delivery of radiofrequency current between sites of early ventricular activation on either side of the septum. This bipolar electrode configuration has been used in eight patients (aged 13-53; six women, two men) with intermediate or posteroseptal accessory pathways (two concealed), two of whom had previously undergone unsuccessful radiofrequency procedures. Ablating catheters ( $4 \mathrm{~mm}$ tip, $2.5 \mathrm{~mm}$ spacing) were introduced into the right and left ventricles and the septal region was mapped. Although favourable electrograms could always be recorded from both sides of the septum, multiple (up to 44) unipolar radiofrequency discharges from right or left ventricles to a surface indifferent electrode were ineffective or only produced transient accessory pathway block. By contrast, bipolar radiofrequency delivery between right and left ventricular electrodes permanently abolished accessory pathway conduction in all eight patients within $10 \mathrm{~s}$ : in five cases bipolar delivery succeeded with the same electrode positions at which unipolar radiofrequency pulses had failed. The first bipolar pulse was effective in five patients, and five to nine bipolar pulses were required in the other three. The time from delta wave onset to local ventricular activation ranged from -15 to $10 \mathrm{~ms}$ at the right ventricular sites and -20 to $0 \mathrm{~ms}$ at the left sites. Local atrioventricular times were $30-60 \mathrm{~ms}$ in the right ventricle (continuous activity in five of eight electrograms) and $20-65 \mathrm{~ms}$ in the left ventricle (continuous activity in four of eight). Accessory pathway conduction has not recurred in any patient during mean follow up of 16 (range two to 22) weeks.

Use of the bipolar right and left ventricular technique may successfully ablate septal accessory pathways where multiple unipolar radiofrequency pulses have been ineffective, and potentially could reduce procedure time, radiation exposure and overall failure rate in these difficult cases.

\section{Detailed mapping of the posteroseptal space for catheter ablation of posteroseptal accessory pathways}

\section{E Rowland, J M Morgan, D T Connelly, C S Nandra,} A D Cunningham

Royal Brompton National Heart and Lung Hospital, London

The posteroseptal space has complex anatomical relations and localising accessory pathways in this region is difficult. Between 1988 and 1991 we have undertaken catheter ablation of posteroseptal accessory pathways in 19 patients (12 men, aged 19 to 57 , mean 35 ). All patients had had palpitation (for seven to 24 (mean 15) years), four had been syncopal during tachycardia (two rescued from sudden cardiac death) and all were identified on the basis of standard electrocardiographic appearances and the finding of earliest activation at the mouth of the coronary sinus during previous routine electrophysiological study. At catheter ablation, localisation was performed during atrioventricular re-entrant tachycardia or ventricular pacing in all, and in 15 where pre-excitation was overt, by measurement of local ventricular electrogram to surface delta in sinus rhythm. Detailed recordings were made from the posterior right freewall, post-eustachian space, septal aspect of the tricuspid annulus, and proximal portion of the coronary sinus. When earliest activation occurred in the coronary sinus left sided mapping on the medial aspect of the mitral annulus (MA) was also performed. Coronary sinus angiograms were also obtained; at the site of the pathway there was a stricture in one case, and a diverticulum in another. Lesions were created using low energy direct current in 16 patients and radiofrequency in three and successfully ablated the pathway in 14 (follow up two to 18 median nine months). The ablation site was the posterior right free wall in one, post-eustachian space in three, tricuspid annulus in one, proximal portion of the coronary sinus in six, and a combined proximal portion of the coronary sinus-mitral annulus approach was required in three patients.

Posteroseptal accessory pathways are not uniformly located despite similar electrocardiographic appearances and routine electrophysiological findings. Detailed mapping of the posteroseptal space is feasible and can identify the site of the pathway with sufficient precision to permit successful catheter ablation in most cases.

\section{Endothelium-derived relaxing factor (EDRF) modulates contraction in the intact heart}

S Fort, A M Shah, M J Lewis

Cardiovascular Research Group (Departments of

Cardiology and Pharmacology and Therapeutics), University of Wales College of Medicine, Cardiff

Endocardial endothelium modulates myocardial contraction in isolated papillary muscle preparations through the release of a novel contraction prolonging agent, "endocardin", and endothelium-derived relaxing factor (EDRF) that reduces contractile duration, neither agent having any major effect on the early phase of contraction. The influence of EDRF on contraction of the intact heart was investigated. Isolated paced ejecting guinea pig hearts were studied at constant filling pressure and afterload (with $1 \mu \mathrm{M}$ indomethacin and $0 \cdot 1 \mu \mathrm{M}$ acebutolol). Bradykinin $(0 \cdot 1 \mu \mathrm{mol} / 1)$, which releases EDRF from vascular endo- 
thelium, shortened the duration of the left ventricular pressure profile without influencing early systole: ejection duration decreased by $6 \%$ and cardiac output by $31 \%$ $(\mathrm{n}=6$, both $\mathrm{p}<0.05)$, with no change in peak left ventricular pressure, end diastolic pressure or peak rate of pressure rise. Coronary effluent nitrite concentrations (a measure of EDRF production) increased from $3.7(0.4)$ to $6.5(0.8) \mathrm{nmol} / \mathrm{min}$, and coronary flow from $7.6(0.9)$ to 9.2 $(1 \cdot 1) \mathrm{ml} / \mathrm{min}$ (both $\mathrm{p}<0.05)$. When EDRF synthesis was inhibited by $30 \mu \mathrm{M} L-N^{G}$ monomethylarginine, all these effects of bradykinin were almost abolished (ejection duration $+0.4 \%$, cardiac output $+1.3 \%$, coronary nitrite $1 \cdot 2(0 \cdot 2)$ to $2 \cdot 2(0 \cdot 3) \mathrm{nmol} / \mathrm{min})$.

These data show that EDRF modulates contractile duration in the intact heart as it does in isolated papillary muscle preparations. They suggest a novel regulation of cardiac pump function by the coronary vascular endothelium.

\section{Endothelium-derived factor(s) reduce contraction amplitude of isolated functioning cardiac myocytes}

A J B Brady, J B Warren, T J Williams,

P A Poole-Wilson, S E Harding

National Heart and Lung Institute, London

Recently, the endothelial lining of cardiac muscle has been shown to modulate contraction of isolated papillary muscle preparations, and the contractility of isolated cardiac myocytes can be reduced by the nitric oxide donor, sodium nitroprusside. Furthermore, 5-hydroxytryptamine releases nitric oxide from the coronary microvasculature. We have developed a novel coculture model of functioning cardiac myocytes sited on confluent endothelial cells to investigate whether nitric oxide from endothelium modifies myocyte contractility. Endothelial cells were prepared non-enzymatically from bovine aorta and cultured on glass coverslips. A coverslip was mounted on the stage of an invertd microscope and superfused with Krebs-Henseleit buffer, containing $2 \mathrm{mM}$ calcium, at $32^{\circ} \mathrm{C}$. Guinea pig cardiac myocytes were prepared, added to the coverslip, and allowed to adhere to the endothelial cell layer. Contraction amplitude and velocity of shortening of electrically stimulated $(0.5 \mathrm{~Hz})$ myocytes were recorded using a videomicroscopy length detection system and analysed by computer signal averaging. Myocyte contraction amplitude was calculated as a percentage of the resting length. Under control conditions myocytes shortened by $5.3 \%(0.6)$ (mean (SEM), $n=12$ ). Subsequently, $100 \mathrm{mM}$ bradykinin, used to generate nitric oxide from the endothelial cells, reduced myocyte shortening by $10.9(3.5) \%(n=12$ experiments; $p<0.03)$. This effect was not found in the presence of $10^{-4} \mathrm{M} L$ nitroarginine methyl ester (L-NAME), an inhibitor of nitric oxide synthesis $(n=7)$. Maximal contraction velocity and relaxation time were not affected. Myocyte contraction was not modified either by the presence of unstimulated endothelium, or by bradykinin alone.

The contraction of cardiac myocytes is modulated by bradykinin stimulated, but not quiescent endothelium. This small effect is abolished by L-NAME, suggesting that the effect is mediated by endothelial cell nitric oxide derived. This interaction may be of importance as there is an intimate relation between the microvasculature and cardiac myocytes.
Modulation of myocardial contraction via activation of $K^{+}$channels by an agent released from coronary vascular endothelium

\section{S Fort, M J Lewis}

Cardiovascular Research Group (Departments of Cardiology and Pharmacology and Therapeutics), University of Wales College of Medicine, Cardiff

The role of the coronary vascular endothelium in the regulation of myocardial contraction was investigated in isolated, externally paced Langendorff perfused ferret hearts. Hearts from pentobarbitone anaethetised ferrets were excised and perfused with Krebs-Henseleit buffer $\left(37^{\circ} \mathrm{C}, 1.25 \mathrm{mM} \mathrm{Ca}{ }^{2+} ; 95 \% \mathrm{O}_{2}: 5 \% \mathrm{CO}_{2}\right.$ ) containing $0.01 \mathrm{mM}$ acebutolol and indomethacin. Left ventricular pressure was recorded via a fluid filled latex balloon. Continuous intracoronary infusion of bradykinin $\left(10^{-6} \mathrm{M}\right)$ induced a transient (two min) reduction in peak pressure of $16 \cdot 2(3 \cdot 6) \%$ and maximum rate of pressure development of $14.7(2.5) \%$ (both $\mathrm{p}>0.05, \mathrm{n}=6$; Student's $t$ test), associated with a fivefold increase in myocardial cyclic guanosine monophosphate (GMP) concentrations ( $n=6$, $\mathrm{p}<0.05$ ). Earlier $30 \mathrm{~min}$ infusion of $\mathrm{N}^{\mathrm{G}}$-nitro $\mathrm{L}$-arginine $\left(10^{-4} \mathrm{M}\right)$, to inhibit release of endothelium-derived relaxing factor, abolished the elevation of myocardial cyclic GMP concentrations but not the inoptropic response to bradykinin. Earlier bolus injection of $0.1 \mathrm{ml} 0.5 \%$ triton $\mathrm{X}-100$, to remove endothelium, abolished both the cyclic GMP and inotropic responses to bradykinin. Earlier five min infusion of the $\mathrm{K}^{+}$-channel blockers glibenclamide $\left(10^{-6} \mathrm{M}\right)$, or 4-aminopyridine $\left(10^{-3} \mathrm{M}\right)$ likewise inhibited the inotropic response to bradykinin.

These results suggest that myocardial contractile performance can be influenced by a coronary endotheliumderived factor that acts by opening myocardial $\mathrm{K}^{+}$ channels.

\section{Mechanism of hypoxic vasorelaxation of precontracted isolated porcine coronary arteries}

Y Butt, T Higenbottam, J Pepke-Zaba, G Cremona, M Takao, A T Dinh-Xuan

Department of Respiratory Physiology, Papworth Hospital, Cambridge

Although hypoxic vasodilatation of coronary arteries may be important in sustaining myocardium distal to stenoses, little is known of the mechanism. To study the phenomena we have used 62 porcine coronary artery rings ( 2 to $5 \mathrm{~mm}$ in diameter), the heart was obtained within five minutes of death from nine animals. Rings were studied in duplicate, one with endothelium $(E+)$ and one where endothelium was mechanically removed $(E-)$. The tension was studied isometrically in $20 \mathrm{ml}$ organ baths containing Krebs-Ringer bicarbonate solution at $37^{\circ} \mathrm{C}$. Initially $95 \% \mathrm{O}_{2}$ and $5 \% \mathrm{CO}_{2}$ gas mixture was continuously bubbled through the solution giving a $\mathrm{Po}_{2}$ of 68.4 $(2.9) \mathrm{kPa}$. The rings were precontracted with prostaglandin $\mathrm{F}_{2 \alpha}\left(10^{-7}\right.$ to $\left.10^{-5} \mathrm{M}\right)$ to achieve a stable plateau of contraction by a mean period of 33 minutes. Then rings were treated with nothing (control); $\mathbf{N}^{\omega}$-nitro-L-arginine methyl ester ( $\mathrm{L}$ NAME) $\left(10^{-4} \mathrm{M}\right)$, which inhibits nitric oxide synthase; ouabain $\left(10^{-6} \mathrm{M}\right)$, which inhibits $\mathrm{Na}^{+}-\mathrm{K}^{+}$-ATPase; tetraethylammonium (TEA) $\left(5 \times 10^{-3} \mathrm{M}\right)$, which inhibits the voltage dependent $\mathrm{K}^{+}$channels or tolbutamide $\left(10^{-4} \mathrm{M}\right)$, which inhibits ATP- $\mathrm{K}^{+}$channels. After achieving a further stable plateau of tension the bubbling gas mixture was 
switched to $95 \% \mathrm{~N}_{2}$ and $5 \% \mathrm{CO}_{2}$ to give a final $\mathrm{Po}_{2}$ of $8 \cdot 8$ $(0.3) \mathrm{kPa}$ and stable relaxation in 46 minutes (range 14-82 $\mathrm{min})$. We have found that hypoxia caused complete relaxation of the control coronary artery rings $(113.2(7 \cdot 0) \%$ and 114.6 $(12.1) \%$ in $\mathrm{E}+$ and $\mathrm{E}-$ rings respectively, NS). In comparison with control $\mathrm{E}+$ rings there was a significant reduction in relaxation of rings treated with ouabain (59.4 $(7.3) \%$ in $\mathrm{E}+; \mathrm{p}<0.001$ and $73.8(11.0) \%$ in $\mathrm{E}-$ rings; $\mathrm{p}<0.01)$, TEA $(79.6(7.5) \%$ in $\mathrm{E}+; \mathrm{p}<0.01$ and 77.2 $(12.9) \%$ in $\mathrm{E}-$ rings; $\mathrm{p}<0.02)$ and L-NAME (79.9 $(11 \cdot 1) \%$ in $\mathrm{E}+$ rings; $\mathrm{p}<0.02)$. There was no significant reduction in hypoxic relaxation of rings treated with tolbutamide (104.5 (13.8)\% in E and $93.4(24.2) \%$ in $\mathrm{E}$ - rings).

Going from hyperoxia to hypoxia causes a comparable relaxation of coronary artery rings with and without endothelium. The predominantmechanism involves vascular smooth muscle $\mathrm{Na}^{+}-\mathrm{K}^{+}$-ATPase and to a lesser degree voltage dependent $\mathrm{K}^{+}$channels. Also, in coronary artery rings with endothelium, release of nitric oxide contributes to relaxation.

\section{Nitric oxide (NO) release is responsible for the accommodation of the pulmonary vasculature to increased flow}

\section{G Cremona, T Higenbottam, A Wood, E A Bower}

Respiratory Physiology Department, Papworth Hospital, Cambridge

It is believed that increased rate of perfusion of the lungs is accommodated by passive recruitment of pulmonary arteries. In large mammals such as humans, sheep, and pigs, nitric oxide is continuously released from pulmonary endothelium and determines the low basal pulmonary arterial tone (Cremona G, et al. Am Rev Respir Dis 1991). Here we describe the effects of inhibition of nitric oxide production the adaptation of the vasculature of isolated lungs to increased rate of perfusion. The lungs were "harvested" from eight anaesthetised pathogen free sheep using a method developed to preserve heart and lungs for transplantation (Hakim $M$ et al, J Cardiovasc Thorac Surg 1989). The lungs were ventilated with $20 \% \mathrm{O}_{2}, 5 \% \mathrm{CO}_{2}$ and perfused through a pulmonary artery cannula with either Krebs-dextran (35\% dextran and 5\% albumin) or autologous blood (mean haematocrit 35\%). A Doppler flow probe was used to monitor flow rate into the pulmonary artery and a perfusion pressure was recorded with a transducer connected to a catheter inserted into the pulmonary artery. The perfusate was allowed to drain freely into the venous reservoir kept at constant temperature $\left(37^{\circ} \mathrm{C}\right)$, the rate of perfusion being altered by varying the speed of the roller pump. Indomethacin $\left(10^{5} \mathrm{M}\right)$ was added to the reservoir in all studies. Blood and Krebs-dextran perfusates were studied with and without pre-treatment with $\mathrm{N}^{(\omega)}$-nitro-L-arginine methyl ester $(\mathrm{L}-$ NAME) $\left(10{ }^{4} M\right)$, which inhibits nitric oxide synthase, and subsequently each was studied after addition of the exogenous nitrovasodilator sodium nitroprusside $\left(10^{4} \mathrm{M}\right)$. A linear relation was found between perfusion pressure and flow rate. The regression of perfusion pressure on flow rate had significantly steeper slopes with blood than Krebs-dextran perfusate (blood 8.7 (0.8); Krebs-dextran $5 \cdot 15(0 \cdot 2)$, this difference in slope was maintained after L-NAME. Inhibition of nitric oxide production with L-NAME significantly increased the intercept value of pressure at which flow started (Krebsdextran $0.8(0.3)$ to $7.9(1)$; blood 3.1 (1) to $8.8(1.5)$. In both Krebs-dextran and blood perfused lungs slopes also increased with L-NAME (Krebs-dextran 10 (0.6); blood 13.6 (1). Sodium nitroprusside causes the curves to return to normal.

These data suggest that active release of nitric oxide is a determinant not only of low pulmonary vascular tone but also of the critical opening pressure of pulmonary arteries recruited by increased flow.

\section{Distribution and regulation of binding sites for ${ }^{125} \mathrm{I}$-calcitonin gene related peptide in bovine coronary arteries}

J Wharton, G A Knock, J A R Gaer, K M Taylor, J M Polak

Royal Postgraduate Medical School, Hammersmith Hospital, London

It is uncertain whether neuropeptides, such as calcitonin gene related peptide (CGRP), exert a significant tonic influence on vascular peptide receptors and if regional variation in the vascular activity of peptides may be due to differences in the distribution of receptors. We have examined the distribution and characteristics of ${ }^{125}$ I-labelled human CGRP ( ${ }^{125} \mathrm{I}$-hCGRP) binding sites in the left anterior descending coronary artery of innervated and denervated bovine hearts. Complete functional denervation of the heart was achieved by a novel cryosurgical technique two to four weeks before excising the heart. Denervation was confirmed by direct nerve stimulation, assaying tissue catecholamine concentrations, and by immunohistochemistry. ${ }^{125}$ I-hCGRP binding sites were localised and analysed using quantitative in vitro autoradiographic techniques. Specific high affinity $\left(\mathbf{K}_{\mathbf{d}}\right.$ $0.39 \mathrm{nM}(95 \%$ CI $0.26-0.52 \mathrm{nM}){ }^{125}$ I-hCGRP binding sites were localised to the media of both epicardial and intramyocardial bovine coronary arteries. A differential distribution of binding was found, with a higher density of binding sites occurring on distal compared with proximal epicardial regions of the left arterial descending coronary artery $(p=0.0041)$ and on intramyocardial compared with epicardial arteries $(p=0.0001)$. The binding sites exhibited a higher affinity for hCGRP (K $0.85 \mathrm{nM}$ (0.51-1.19) than for hCGRP $_{8-37}\left(\mathrm{~K}_{\mathrm{i}} 7.49 \mathrm{nM}\right.$, $(4 \cdot 22-10 \cdot 76)$ ) and the linear analogue (Cys (ACM) ${ }^{2 \cdot 7}$ ) hCGRP $\left(K_{\mathrm{i}} 30.44 \mathrm{nM},(20.84-40.04)\right)$, suggesting that they correspond to the $C_{G R P}$ receptor subtype. Addition of guanosine-5-O-(3-thiotriphosphate) to the incubation buffer reduced the association and increased the dissociation of ${ }^{125} \mathrm{I}-\mathrm{hCGRP}$ binding, indicating that the binding sites are coupled to a guanine nucleotide binding protein. Extrinsic cardiac denervation resulted in a depletion of CGRP-like immunoreactive nerve fibres associated with coronary arteries and an increase in the density $(p=0.0092)$, but not in the affinity of coronary artery ${ }^{125}$ I-hCGRP binding sites.

The CGRP released from local nerve terminals may exert a tonic influence on coronary artery receptors, and regional variation in the vasodilatory activity of CGRP may reflect a differential distribution of binding sites. It has yet to be determined whether the changes found in binding site density after extrinsic cardiac denervation have a functional consequence. 
Radionuclide angiography with a multiwire camera and tantalum-178: an advance over conventional imaging techniques

\section{S R Vallely, N P S Campbell, J D Laird, R Ferguson, W McNair \\ Royal Victoria Hospital, Belfast}

This investigation assesses the use of a portable, high speed digital multiwire camera and tantalum-178, a short lived isotope $\left(\mathrm{t}^{\frac{1}{2}}=9.3 \mathrm{~min}\right)$ with significantly improved patient dosimetry over conventional agents, for radionuclide angiography. Tantalum-178 is obtained by elution of a generator system housed within the camera and containing the parent isotope tungsten-178 $\left(\mathrm{t}^{\frac{1}{2}}=22\right.$ days) creating a self contained portable imaging system that can be taken to the patient's bedside or to the cardiac catheterisation laboratory in the acute setting. The properties of the camera isotope combination permit the use of larger doses of isotope than with conventional systems while simultaneously decreasing the effective patient radiation dose. As part of the initial assessment of the system, 27 patients underwent supine measurement of ventricular function at rest by gated blood pool imaging with a single crystal camera and technetium- $99 \mathrm{~m}$, and by first pass angiography using a multiwire camera and tantalum-178. Also, 17 of these patients had first pass angiography using the single crystal camera and technetium-99m. Each patient had all investigations performed within a period of two weeks and with no change in medication. Left ventricular ejection fractions using the multiwire system showed a significant correlation with those obtained by gated blood pool imaging $(r=0.86, p<0.001)$, and with those obtained by conventional first pass angiography $(r=0.69$, $p=0.002$ ). The correlation coefficient between the conventionally obtained gated blood pool and first pass images was $0.74(p<0.001)$. The average dose of isotope with the multiwire system was $969 \mathrm{MBq}$ and with the conventional system $555 \mathrm{MBq}$. With the multiwire camera the effective radiation dose was decreased by a factor of 11.4. Average counts in the left ventricular end diastolic frame increased by a factor of 15 with the multiwire camera when compared with conventional first pass angiography (from 374 to 5694).

The multiwire system accurately assesses ventricular function while offering major advantages in terms of improved patient dosimetry.

\section{Assessment of myocardial viability by ${ }^{15}$ O-labelled water and positron emission tomography (PET) in patients successfully thrombolysed for acute myocardial infarction}

R de Silva, Y Yamamoto, C G Rhodes, H Iida, E Rechavia, L I Araujo, P Nihoyannopoulos, D Hackett, T Jones, A Maseri

Medical Research Council Cyclotron Unit, Hammersmith Hospital, London

The study aim was to predict the improvement in regional wall motion after successful thrombolysis for an acute myocardial infarction by positron emission tomography (PET). Eleven patients treated with intracoronary thrombolysis were studied in whom vessel patency was confirmed at angiography 24 hours later. Echocardiography and PET studies were performed three to six days after acute myocardial infarction, at which time 14 asynergic segments were identified. Eleven control segments were selected on the basis of having normal wall motion and an angiographically normal arterial supply. Follow up echocardiography was performed four to six months later to assess contractile recovery. The PET imaging protocol consisted of a transmission scan, ${ }^{15} \mathrm{O}$-labelled carbon monoxide (blood volume) and ${ }^{15} \mathrm{O}$ water emission scans. Regional myocardial blood flow was calculated from the ${ }^{15} \mathrm{O}$ water data set by a previously validated protocol. Manipulation of the above PET data enabled calculation of the perfusable tissue index (PTI) for each myocardial region of interest. The PTI is defined as that proportion of the myocardium within a region of interest that is perfusable by water, and may therefore represent an index of tissue viability. Follow up echocardiography identified $9 / 14$ recovery and 5/14 non-recovery segments. Myocardial blood flow in control regions was 1.02 $(0.24) \mathrm{ml} / \mathrm{min} / \mathrm{g}$, but was reduced to a similar degree in both the recovery and non-recovery segments $(0.68(0.32)$ and $0.60(0.18) \mathrm{ml} / \mathrm{min} / \mathrm{g}, \mathrm{p}<0.02 v$ control $)$. The PTI in the control segments was $0.98(0.08)$, indicating that all the myocardium in these regions was perfusable by water. In the non-recovery regions, PTI was $0.53(0.11)(p<0.02 v$ control), whereas in the recovery segments PTI was relatively preserved $(0 \cdot 88(0 \cdot 10)$, NS $v$ control $)$. In the recovery regions, PTI was always $>0.7$ indicating that at least $70 \%$ of the myocardium should be perfusable by water to enable contractile recovery.

These data indicate that myocardial blood flow alone is not suitable for assessment of myocardial viability and that PTI may represent a valuable means of predicting improvement in regional wall motion after successful thrombolysis.

\section{Myocardial viability after $Q$ wave infarction: evaluation using early and late thallium reinjection imaging}

E Prvulovich, S R Underwood, D E Jewitt King's College Hospital, Denmark Hill, London; and Royal Brompton Hospital, London

Reinjection of thallium after stress and redistribution tomography, improves the detection of viable myocardium in patients with chronic coronary artery disease and left ventricular dysfunction. The optimal time for imaging after reinjection is unknown because of slow redistribution to ischaemic myocardium. To evaluate the potential of thallium reinjection after acute infarction, and to compare early with late reinjection imaging, 30 patients (age 57 (10) y 35 (7) days after a first transmural myocardial infarction were assessed. All patients underwent exercise thallium tomography with image acquisition immediately after stress, after four hours redistribution, and immediately after reinjection of thallium at rest. In a subset of 15 patients, images were also acquired three hours after reinjection. Nine segments per patient were analysed with a visual grading score of tracer uptake from 1 for absent to 5 for normal. One hundred and twenty nine of 270 segments had stress perfusion defects. Of these, $88(68 \%)$ were fixed, and $41(32 \%)$ showed redistribution at four hours before reinjection. Forty two of the fixed defects $(48 \%)$ showed an improvement immediately after reinjection. Delayed imaging after reinjection provided additional evidence of reversible ischaemia in only $9 / 145$ $(6 \%)$ segments.

Imaging early after reinjection detected reversible ischaemia not identified by conventional imaging in 21/30 $(70 \%)$ patients, and provided the only evidence of reversible ischaemia in five $(17 \%)$ patients. These findings have important implications when revascularisation is considered. Late imaging after reinjection, although theoretically attractive, is unwarranted. 
Differences in regional cardiac autonomic innervation detected by metaiodobenzylguanidine imaging

\section{F Shakespeare, C J Page, M J O'Doherty, T O}

Nunan, I C Cooper, D J Coltart, M M Webb-Peploe Cardiac Department, St Thomas's Hospital, London

Metaiodobenzylguanidine (MIBG) is a noradrenaline analogue that can be used to determine cardiac sympathetic innervation. Cardiac sympathetic dysfunction is thought to be a mechanism accounting for the absence of symptoms in silent ischaemia. We aimed to determine if there are regional differences in cardiac sympathetic innervation and whether it is reduced in silent ischaemia. Patients with angiographic evidence of coronary artery disease and no evidence of infarction were allocated to silent and symptomatic ischaemic groups, on the basis of detectable Holter ischaemia with or without symptoms. Patients with Holter silent ischaemia (group $1, \mathrm{n}=6$ ), Holter symptomatic ischaemia (group $2, \mathrm{n}=6$ ), and normal controls $(n=6)$ underwent ${ }^{123}$-MIBG imaging by planar and single photon emission tomography (SPECT) at four hours. Visual analysis of cardiac planar images was also performed. Cardiac uptake was normalised for liver and quantified per short axis area at the base, mid-ventricle and apex. From base to mid-ventricle, uptake increased in group $1(\mathrm{p}<0.006)$, group $2(\mathrm{p}<0.01)$, and controls $(p<0.004)$. From mid-ventricle to apex, uptake increased significantly only in the controls $(p<0.001)$. Visual analysis showed greater anterior than inferior ventricular wall uptake in 14 patients. In group 1 , anterior ventricular uptake was greater than inferior uptake at the midventricle $(p<0.03)$ and apex $(p<0.05)$. In group 2 , the same relation was shown at mid-ventricle $(\mathrm{p}<0.01)$ and apex $(\mathrm{p}<0.05)$. Group 2 patients had a trend towards greater uptake than group 1 , significant only at the midventricle $(p<0.05)$.

These data suggest that sympathetic innervation is greatest in the mid-ventricle and the apex of the left ventricle. Innervation is also greater in the anterior compared with the inferior. There is indirect evidence of reduced sympathetic innervation in silent ischaemia.

\section{Combined assessment of left ventricular wall motion, ejection fraction, and acute myocardial necrosis with a single dose of technetium-99m antimyosin antibody}

R Senior, S Bhattacharya, W Wegener, P Manspeaker, $\mathrm{H}$ Weisman, A Lahiri

Northwick Park Hospital, Harrow, Middlesex

In a prospective study of 26 patients with first $Q$ wave acute myocardial infarction, infarct avid imaging, and radionuclide ventriculography, were performed after injection of technetium-99m (740 MBq) labelled antimyosin within 72 hours of chest pain. The early blood phase of technetium-99m labelled antimyosin was used for equilibrium radionuclide ventriculography in the three standard views. Planar images for the detection of myocardial necrosis were obtained earlier, and at 24 hours, in the same three views. Images were evaluated blind by two experienced physicians. Myocardial antimyosin uptake was seen in 21 out of 26 patients $(81 \%)$. Radionuclide ventriculography was evaluated in 19 out of 21 patients with positive uptake of antimyosin. Mean (SD) left ventricular ejection fraction was $42(9) \%$ (range $25 \%-59 \%$ ). Wall motion abnormality was present in all the 19 patients and this matched with the location of antimyosin uptake. Seven $(47 \%)$, however, had wall motion abnormality extending beyond the area of antimyosin uptake which may represent stunned or hibernating myocardium. In a further four $(21 \%)$ patients, the extent of antimyosin uptake exceeded the area of wall motion abnormality, which may suggest myocardial viability in the area of injury. These data suggest that with a single dose of technetium-99m antimyosin, simultaneous assessment of left ventricular function, and delineation of the extent and location of myocardial necrosis may be achieved, improving prognostic evaluation in patients with acute myocardial infarction.

\section{Technetium-99m tetrofosmin (P53) myocardial perfusion imaging in coronary heart disease: comparison with thallium-201 imaging}

B S Sridhara, R Itti, S Bratt, P Rigo, P Cload, U Raval, D Hinge, A Lahiri

Northwick Park Hospital, Harrow, Middlesex

Technetium (Tc)-99m Tetrofosmin (Amersham International) is a lipophilic , cationic diphosphine (technetiumD) that has been developed for myocardial imaging. We have compared Tc-D with thallium (Tl)-201 imaging for the detection of coronary heart disease, and assessed redistribution characteristics of Tc-D. Twenty three patients were injected with Tc-D during bicycle exercise and at rest four hours later. A thallium-201 exercise and redistribution study was also performed. Multiple planar views were obtained at five $\mathrm{min}, 30 \mathrm{~min}$, two $h$ and four $h$ after injection, and all images were assessed by a blinded panel to score patients with myocardial ischaemia, infarction, or normal scan interpretation. Quantitative defect to normal wall ratio was generated to assess redistribution with technetium-D. Thallium-201 detected myocardial infarction in 13 and reversible ischaemia in nine and a normal scan was found in one patient. Technetium-D detected all correctly except one patient with ischaemia at five min. There was no statistical difference between defect: normal wall ratio between different time points. There was significant increase in lung uptake of thallium-201, but not technetium-D during exercise. Excellent quality diagnostic images were noted as early as five min and up to four $h$, and there was no evidence of any redistribution with technetium-D. Technetium-D compares well with thallium-201 for the diagnosis of coronary heart disease.

The physical characteristics, low cost, and availability of technetium-ligands make them more attractive for myocardial imaging. Thus technetium-D is a unique, new agent for evaluation of patients with coronary heart disease. 
Self-expanding stents for diseased saphenous vein
coronary artery bypass grafts

J T Stewart, M G Williams, J-J Goy, L Denne, N P Buller, A F Rickards, U Sigwart

Royal Brompton National Heart and Lung Hospital, London

Dilatation of stenotic segments of saphenous vein coronary artery bypass grafts by conventional balloon angioplasty is associated with a high restenosis rate, which may be more than $60 \%$ for lesions involving the proximal graft segment. Intravascular stents may reduce restenosis rates in both native coronary arteries and coronary artery bypass grafts. We have attempted implantation of 91 self-expanding stents into 66 coronary artery bypass grafts in 58 patients (mean age 61) during 68 separate procedures. One stent was implanted in 33 patients, two stents were implanted in 17 patients, and three stents in eight patients. Stent diameter ranged from 3.5 to 6.0 (mean 4.7 ) $\mathrm{mm}$ and stent length from 15 to 30 (mean 24.2) mm. Most stents (62\%) were implanted in the body of the graft, but $29 \%$ were implanted in the proximal segment, frequently involving the aortic anastamosis. There was failure to deploy the stent in one of the 68 procedures, a procedural success rate of $98.5 \%$, although implantation was difficult in a further nine cases $(13 \%)$. In hospital occlusion of the stent requiring disobliteration occurred in two cases, with $Q$ wave infarction in one. Bleeding from the femoral puncture site of sufficient severity to warrant transfusion with or without surgical repair occurred after five procedures $(7.5 \%)$. There was one in hospital sudden death in an elderly patient with poor left ventricular function, and four late deaths ( $>3$ months). Necropsy of two of these four patients showed stent patency. During long-term follow up (mean 11 months) restenosis or occlusion was shown in seven $(11 \%)$ of stented vessels in seven patients $(12.3 \%)$, with evidence of myocardial infarction in one. Four patients $(7 \%)$ have undergone repeat intervention (angioplasty or graft).

Use of the self expanding stent increases the scope of non-surgical treatment of diseased coronary artery bypass grafts, is associated with an acceptable complication rate, and has a much lower restenosis rate than conventional percutaneous transluminary coronary angioplasty.

\section{Experience with the Palmaz-Schatz intracoronary stent}

\section{J W Dean, S W Davies, J Clague, M McCool,} M T Rothman

Cardiac Department Royal London Hospital; and London Chest Hospital, London

Intracoronary stents are increasingly used to treat acute dissection and prevent restenosis after percutaneous coronary angioplasty. We have used the Palmaz-Schatz balloon expandable coronary stent in 30 patients. Indications were acute dissection 11 (nine angioplasty, two diagnostic angiography), restenosis seven, vein graft stenosis seven, poor angioplasty result three, ostial stenosis one, and chronic dissection one. Seven were rescue or emergency procedures. A good angiographic result was achieved in all patients but nine technical problems occurred in eight patients; balloon rupture three, vessel dissection proximal to stent site two, thrombosis requiring streptokinase one, misplaced stent two, and dropped stent in coronary artery one. Early complications were few, one patient developed major femoral haematoma due to a displaced collagen plug, and one patient required bypass grafting for acute closure of an undilated vessel. During follow up (0.5-14 months) there have been three deaths; one rescue case died after two days, the other two patients occluded their only supply vessel having had previous restenosis. Twenty three of 27 patients remain asymptomatic, the four symptomatic patients developed restenosis; seven patients had no restenosis at routine reangiography at six months.

Thus the Palmaz-Schatz coronary stent may be used safely in selected cases, producing excellent angiographic results. We have had no significant acute stent related complications. Late complications are usually heralded by symptoms, whereas asymptomatic patients have had no angiographic restenosis.

\section{Temporary stenting as a bale out procedure during coronary angioplasty}

J S R Gibbs, I A Simpson, J E Nordrehaug, A F Rickards, U Sigwart, N Buller

Royal Brompton National Heart and Lung Hospital, London

Autoperfusion balloon catheters that are used to maintain coronary perfusion after inadvertent coronary dissection during coronary balloon angioplasty may limit distal coronary perfusion and obstruct side branches. We have evaluated a temporary stent (Flow Support Catheter, ACS), which provides an internal scaffold to the artery wall when deployed. A radiopaque, braided wire cage, $30 \mathrm{~mm}$ in length, and permanently affixed to the distal end of the shaft can be expanded up to a maximum diameter of 4.0 $\mathrm{mm}$ and collapsed via a manipulator on the proximal end of the catheter. The shaft of the catheter is a 3.7 French rapid exchange design, allowing the catheter to be deployed over the guide wire. Four patients (age range 41-69) who developed acute coronary dissection during routine balloon angioplasty underwent temporary stenting as an emergency bale out procedure to restore coronary blood flow. Angioplasty was being performed to the left anterior descending coronary artery in three patients and right coronary artery in one patient. Before the temporary stent expansion, flow distal to the dissection was thrombolysis in Myocardial Infarction Trial (TIMI) grades 0-2. During stent expansion (30-209 min) TIMI 3 flow was maintained to both the artery and its side branches covered by the stent. In one patient the device resulted in successful treatment of the dissection, two patients required permanent stenting after its use and in one patient it was used as a bridge to coronary artery surgery. No patient developed enzymatic or electrocardiographic evidence of myocardial infarction after the procedure.

Although worldwide experience of this device is limited, the temporary stent was simple to use and maintained good coronary artery flow while perfusing side branches. The rapidity with which it can be deployed, and its ease of use make it a useful bale out technique and bridge to other definitive treatment. 
Results of stent implantation for the management of aorto-ostial stenosis

\author{
J E Nordrehaug, N Chronos, L Denne, A F Rickards, \\ N Buller, U Sigwart \\ Royal Brompton National Heart and Lung Hospitals, \\ London
}

Angioplasty of stenosis in the ostium of the right coronary artery and saphenous vein grafts carry a high rate of complications and restenosis. These may be reduced by the use of intravascular stents. We report the results of aortoostial stenting in 10 patients (nine men), mean age 61 (8). Eight self expanding stents were implanted in saphenous vein grafts and two were implanted in the right coronary artery. Nine were elective procedures. The mean stent length was $27.6(6 \cdot 1) \mathrm{mm}$ (range 20-40) and the diameter $5.0(0.65) \mathrm{mm}$ (range $4 \cdot 0-5 \cdot 5)$. One patient had a slight enzyme rise after procedure and none developed $Q$ wave infarction. There were no in hospital occlusions. A satisfactory primary angiographic result was obtained in all patients. The mean follow up was 16 (12) months (range 4-38). During this time five patients remained symptom free and three others were improved compared with before the procedure. The five symptomatic patients underwent repeat angiography. Of these, two had developed stent restenosis and three had new lesions in other vessels requiring intervention. One patient died from a non-stent related cause.

These preliminary data suggest that stent implantation may be a useful strategy for the management of aorto-ostial stenosis.

\section{Containment of dissection after percutaneous transluminal coronary angioplasty by an implantation of a Palmaz-Schatz coronary artery stent}

\section{S P Verma, M R Rees, U M Sivananthan, P Thorley,} I Sabroe

Departments of Cardiology, Cardiac Radiology and Cardiac Research Unit, Killingbeck Hospital, Leeds

Twenty two Palmaz-Schatz coronary stents were implanted in 20 patients ( 16 men, four women; mean age 53 (range 48-76) to contain dissection after percutaneous transluminal coronary angioplasty (dissection compromising flow 14, dissection with acute occlusion four, dissection with thrombosis two). Percutaneous transluminal coronary angioplasty was performed (left anterior descending coronary artery 13, right coronary artery 7) for severe angina (rest pain in 10, severe exertional angina with positive stress test in five, and chest pain and reversible thallium defect in five). The coronary artery disease comprised 14 stenoses, four chronic occlusions, two stenoses with occlusion (dissection of a normal vessel occurred in one of these). Dissection was successfully contained in 14 patients (one stent in 12 patients, two stents in two), restoring flow: in the remaining six, failure to deliver a stent occurred in two patients, and failure to contain dissection in the other four (three required emergency coronary artery bypass graft). A stent occlusion occurred in five patients (within 24 hours in three, at day five in one, and at day 10 in one) one of whom sustained a myocardial infarction.

Although in most patients, flow threatening dissection after percutaneous transluminary coronary angioplasty can be contained with Palmaz-Schatz coronary artery stents, early occlusion may present a serious problem.

\section{Percutaneous cardiopulmonary support in resuscitation}

\author{
T Browne, U M Sivananthan, M R Rees, S P Verma, \\ G A Davies
}

Departments of Cardiac Radiology, Cardiac Surgery and the Cardiac Research Unit, Killingbeck Hospital, Leeds

Percutaneous cardiopulmonary support has a significant role in the emergency situation. Percutaneous cardiopulmonary support renders haemodynamic stability and maintains perfusion to vital organs thereby enabling intervention to be carried out. Emergency percutaneous cardiopulmonary support was instituted in eight patients (seven men, one woman; mean age 66 range (54 to 82)). Four were in asystole and four were in cardiogenic shock, all after cardiac arrest. Six underwent coronary angiography and subsequent coronary angioplasty, two patients also had intracoronary streptokinase. In two other patients percutaneous cardiopulmonary support was instituted, one after cardiac surgery who could not be removed from bypass, and the other after prolonged resuscitation. Treatment was discontinued as they remained unresponsive with severe left ventricular dyskinesia. In all patients vascular access was obtained by percutaneous cannulation of the femoral vessels using $17 \mathrm{f}$ cannulae. The portable percutaneous cardiopulmonary support system consists of a non occlusive vortex pump, heat exchanger, hollow fibre oxygenator, and a control console. Three of the six patients who underwent emergency supported angioplasties survived and were discharged from hospital.

Emergency percutaneous cardiopulmonary support provides a powerful resuscitation tool after which intervention can successfully be undertaken.

\section{Major risk factors for ischaemic heart disease in type 1 (insulin dependent) diabetes: a study of identical twins}

S Dubrey, M Seed, D Lane, H Ireland, M O'Donnell, D Reevely, B O'Connor, R D G Leslie

Academic Unit of Cardiovascular Medicine, and Department of Medicine, Westminster Hospital, London

The major cause of excess mortality in insulin dependent diabetes is cardiovascular disease. Potential risk factors for this excess mortality include blood pressure, body mass index, smoking, lipids, lipoproteins, and coagulation factors. Many of these factors are subject to powerful genetic control. Therefore, to establish whether these risk factors are altered by diabetes we studied identical twins discordant for insulin dependent diabetes. Forty five pairs of twins (24 male pairs), mean (SD) age 38 (11) years, mean disease duration 17 (8) years, were selected because they were aged 25 to 85 and without antihypertensive treatment or significantly increased albumin excretion rates, serum creatinine, or hypertension. Non-fasted blood was tested for all risk factors including lipoprotein (a) by ELISA (enzyme linked immunosorbent assay), and apolipoproteins (apo), fibrinogen, and factor VII by nephelometric methods. As expected, diabetic twins had significantly higher blood glucose and glycated haemoglobin compared with their non-diabetic co-twins ( $p<0.0001)$. Frequency of cigarette smoking was similar in diabetic $(33 \%)$ and non-diabetic $(40 \%)$ twins. Genetic influences were probably important for all other risk 
factors as they were strongly correlated between twins for blood pressure (systolic and diastolic), body mass index, cholesterol, high density lipoprotein cholesterol, low density lipoprotein cholesterol, triglyceride, apo A1 and B, lipoprotein (a), factor VII, and fibrinogen concentrations ( $p<0.01$ in all cases). Apo (a) isoforms were identical in twin pairs. There were no significant differences between diabetic and non-diabetic twins for body mass index, diastolic blood pressure, cholesterol, low density lipoprotein cholesterol, triglyceride, apo B, lipoprotein (a), and factor VII concentrations. By contrast, four variables were significantly higher in diabetic twins compared with their non-diabetic co-twins-namely, systolic blood pressure $(p<0.01)$, high density lipoprotein cholesterol (p < 0.05), apo Al $(\mathrm{p}<0.05)$, and fibrinogen $(\mathrm{p}<0.05)$. There was no change in risk factor profile when only 34 twin pairs without retinopathy or microalbuminuria were considered.

The degree of heritability of these cardiovascular risk factors is considerable in patients with diabetes, but diabetes itself adversely affects only blood pressure and fibrinogen concentration.

\section{Snooze-induced excitation of sympathetic triggered activity (SIESTA): the effects of an afternoon nap on the circadian patterns of heart rate and blood pressure}

\section{Mulcahy, C Wright, D Curcher, D Cunningham,} J Sparrow, H Purcell, K M Fox

Royal Brompton National Heart and Lung Hospital, London

The surge in ischaemia and onset of myocardial infarction in the morning hours is particularly related to the period after waking and commencing activities. It is suggested that the surge in heart rate and blood pressure at this time is associated with these events. Many people have a siesta in the afternoon (an assumed healthy practice); however, it is not clear whether similar haemodynamic changes occur after such a practice. We performed forty eight hours of ambulatory heart rate and blood pressure monitoring on two consecutive working days in 10 healthy subjects. After a pasta and wine (two glasses) lunch (12 30 hours), subjects were randomised to return immediately to work (1330 hours) or to a two hour siesta (1330 hours) lying in a dark room before work, and to alternate the next day. Subjects performed similar activities each evening after work. Heart rate (max, min, mean) was recorded every five minutes during the two days, and blood pressure every 10 minutes from 0600 to 2200 hours and hourly from 2200 to 0600 hours. Circadian patterns were constructed for rate pressure product in addition to individual variables. The rate of change of heart rate and blood pressure after a siesta was similar to that recorded after waking and commencing activities in the morning, and followed appreciable drops in both parameters during the siesta.

It is possible that for those with coronary artery disease, the sudden changes in the determinants of myocardial oxygen demand after a siesta may act as a trigger for cardiac events, much as has been shown in the early hours after waking in the morning.
Dissociation of coronary stenoses progression from changes in left ventricular function in chronic stable angina pectoris

D Tousoulis, T Crake, D Lefroy, J C Kaski, A W Haider, A Maseri

Cardiovascular Unit, Hammersmith Hospital, London

Although left ventricular function is a major prognostic factor in patients with chronic stable angina, it is uncertain whether left ventricular function deteriorates in the absence of acute myocardial infarction. We studied twenty eight patients with stable angina and angiographically documented coronary artery disease who underwent repeat angiography after a mean (SD) interval of 27.3 (1.3) months. Ejection fraction and percentage severity of stenosis were measured on the first and second angiograms by a computerised system. Stenosis progression was defined as an increase of $\geqslant 20 \%$ diameter reduction in a pre-existing stenosis of $\geqslant 50 \%$, or an increase of $30 \%$ in a pre-existing stenosis $<50 \%$, or any increase in lesion severity that resulted in total coronary occlusion, or a new lesion at second angiography $\geqslant 50 \%$. Fifteen of $86(17 \cdot 4 \%)$ stenoses progressed in 12 patients, (in nine of these $(32 \%$ ) to total or subtotal occlusion). Ejection fraction was unchanged at the time of the first and second angiograms both in patients with stenosis progression and in those without $(70 \cdot 1(2 \cdot 2) \% v 67(3) \%$ and $68.3(1.8) \% v 67.8$ $(2 \cdot 1)$, respectively). In patients with stenosis progression the heart rate blood pressure product at $1 \mathrm{~mm} \mathrm{ST} \mathrm{segment}$ depression during exercise testing, was similar at the time of first and second angiography $\left(204(26) \times 10^{2}\right.$ and 193 (29) $\times 10^{2}$ beats $/ \mathrm{min} \times \mathrm{mm} \mathrm{Hg}$ respectively). Increased collateral circulation (grade 3 according to Rentrop scoring) was found in the second angiogram of nine patients who developed total coronary occlusion compared with the first.

In patients with chronic stable angina, coronary stenoses may progress even to total occlusion without clinical deterioration, modification of exercise capacity, or change in the resting left ventricular function. Development of collateral vessels may contribute to the preservation of left ventricular function in patients in whom stenoses progress to total coronary occlusion.

Is life-long anti-arrhythmic therapy necessary to prevent ventricular tachycardia after myocardial infarction?

S K Jain, R D Levy, N H Brooks, D H Bennett Cardiothoracic Centre, Wythenshawe Hospital, Manchester

It is not known whether it is necessary to continue amiodarone long-term to prevent ventricular tachycardia that has occurred $>10$ days after a myocardial infarction. This question is important because of the high incidence of side effects of amiodarone treatment. In this pilot study, amio ( $200 \mathrm{mg}$ once daily, $\mathrm{n}=7400 \mathrm{mg}$ once daily $\mathrm{n}=1$ ) was withdrawn from eight patients, (mean age 63; seven men (range 56-71) after mean 72 (range 46-108 months). Patients were on no other antiarrhythmic drugs and were assessed by electrocardiography, ambulatory monitoring, exercise testing, signal averaged electrocardiogram (SAECG), electrophysiological testing, and drug concentrations. The electrocardiogram showed left bundle branch block in two patients. Left ventricular ejection 
fraction, assessed by echocardiogram, was $<30 \%$ in three and $>30 \%$ in five patients. Ambulatory monitoring showed no VT before stopping amio, at six weeks or at six months after cessation of treatment. Exercise duration at six weeks increased by mean $44 \%$ (range 25-90). All had late potentials on SAECG at entry, persisting at six weeks and at six months after stopping treatment. High frequency low amplitude and mean root mean square voltages were unchanged, but QRS duration (ms) shortened between six weeks (unfiltered 129 R103-144, filtered 157 R143-172) and six months (unfiltered 108 R91-126, filtered 135 R120-149). Electrophysiological testing was performed at six weeks $(n=6)$ and at six months $(n=4)$. Up to two extrastimuli were used, drive cycle length 600 and $450 \mathrm{~ms}$ at the right ventricular apex and in the right ventricular outflow tract. At six weeks, ventricular tachycardia was not inducible in five patients and the ventricular effective refractory period (VERP) was a mean $265 \mathrm{~ms}(\mathrm{R} 240-300)$ at drive cycle length $600 \mathrm{~ms}$. One patient had sustained ventricular tachycardia, was treated with sotolol, and was subsequently non-inducible. At six month mean VERP was $240 \mathrm{~ms}$ (R190-260). Amiodarone concentration was $1 \cdot 1(0.47) \mathrm{mg} / \mathrm{l}$ at entry $(\mathrm{n}=8), \quad 0.45 \quad(0.27)$ at 6 weeks $(\mathrm{n}=6)$ and $0.15(0.13)(n=4)$ at six months. Desethylamiodarone concentration was $1 \cdot 1(0.50), 0.67(0.39)$, and $0.16(0.16)$ respectively. Sleep disturbances were abolished and photosensitivity improved. Thyroid and liver function tests returned to normal. One patient with three previous myocardial infarctions died suddenly at 24 weeks with a fresh myocardial infarction.

These preliminary data show that not all patients with sustained ventricular tachycardia that occurred more than 10 days after a myocardial infarction require life-long antiarrhythmic therapy.

\section{Hypersensitivity reactions to streptokinase in patients with high pretreatment antistreptokinase antibody and neutralisation titres: experience with 189 consecutive patients with suspected myocardial infarction}

H S Lee, S Yule, A McKenzie, S J Cross, T Reid, R Davidson, K P Jennings

Departments of Cardiology, Immunology and

Haematology, Aberdeen Royal Infirmary, Aberdeen

Antistreptokinase antibody $(\mathrm{AB})$ and neutralisation titres (NT) can be raised for at least four years after previous streptokinase. No previous study has shown the association of these antibodies to hypersensitivity reactions with streptokinase. We measured pretreatment $\mathrm{AB}$ and NT in 189 consecutive patients (mean age (SD) 59 (9.8); 130 men) with suspected myocardial infarction. One hundred and three patients $(54.5 \%)$ had confirmed myocardial infarction. Fifty seven patients were treated with streptokinase or anistreplase. One hundred and eighty two patients had not received previous streptokinase (mean (SEM) AB, 25 (3), NT, $0 \cdot 14(0.08) \mathrm{MU}$ ) and seven had (AB 801 (332), NT 3.3 $(1 \cdot 7) \mathrm{MU})$. Nine of the $182(4.95 \%)$ without previous streptokinase and anistreplase had a raised $\mathrm{AB}$ titre of 160 and NT $\geqslant 0.3 \mathrm{MU}$. Three patients had documented hypersensitivity reactions to streptokinase. All of these had raised levels of AB and NT. One patient had acute facial flushing and dizziness minutes after streptokinase infusion. His systolic blood pressure later dropped to $70 \mathrm{~mm} \mathrm{Hg}$ (admission $130 / 70 \mathrm{~mm} \mathrm{Hg}$ ). He received hydrocortisone and chlorpheniramine before streptokinase. His prestreptokinase $A B$ was 160 and NT was $0.48 \mathrm{MU}$. The second patient (AB 160, NT 1.1 MU) did not infarct but received streptokinase. He developed serum sickness with fever, pleural rub, and raised liver enzymes. The third patient's streptokinase was stopped after $150000 \mathrm{U}$ with a blood pressure drop to $70 / 40 \mathrm{~mm} \mathrm{Hg}$ (AB 160, NT 1.5 $M U)$. She developed complete heart block and died from cardiac failure. It is possible that she had an allergic reaction to streptokinase. No patients with low AB or NT had documented reactions with streptokinase. One patient received streptokinase nine months previously, he reinfarcted and received a further dose of streptokinase. His pre-treatment concentrations of both $A B$ and NT were low (AB 10, NT 0.13 MU). He did not suffer an allergic response to the second dose of streptokinase.

High pre-treatment $\mathrm{AB}$ and NT are associated with hypersensitivity reactions to streptokinase or anistreplase.

\section{Complications of epidural spinal electrical stimulation (ESES) for angina}

J E Sanderson, B Ibrahim, D Waterhouse,

R B G Palmer

Department of Cardiology, Taunton and Somerset Hospital, Taunton

We have implanted epidural spinal electrical stimulation units in 23 patients (aged 46-81) with angina unresponsive to standard medical and surgical treatment. Follow up has been for nine to 50 months. Symptomatic improvement was significant with a change of New York Heart Association grade from a mean $3(0.75)$ to $1.85(0.75)$ and glyceryltrinitrate consumption fell. These effects were maintained over the follow up period. There were, however, significant complications: (a) electrode displacement occurred in 16 patients. Six different types of electrodes were used; the Sigma long displaced eight times in six patients. A new electrode with a spiral S-shape and tines has not displaced (seven patients). (b) Fractures of insulation or core occurred nine times in three patients. (c) Infections at the site of stimulator implant developed in three and a superficial infection at the site of epidural insertion in one patient. No infections of the epidural space occurred. (d) New stimulator units were needed in five because of end of life (one), infection (one), programming failure (two), and elective (one). (e) Voltage adjustments and reprogramming were needed in eight patients. (f) Two patients died during the follow up period; one during angioplasty and one from cardiogenic shock after a myocardial infarction.

Thus epidural spinal electrical stimulation is a useful technique for the occasional patient with continuing anginal pain despite standard medical and surgical treatments. It appears to be safe but there are complications, with a high incidence of lead displacements. New lead designs may overcome this particular problem.

\section{Reduction of global ventilation-perfusion mismatch is related to exercise capacity in chronic heart failure}

N G Uren, S W Davies, A G Irwin, S L Jordan, A J W Hilson, J E Agnew, D P Lipkin Royal Free Hospital, London

Increased mismatch of ventilation to lung perfusion $(\mathrm{V} / \mathrm{Q})$ has been suggested as a possible mechanism for the 
disproportionate increase in minute ventilation to carbon dioxide production $\left(\mathrm{VCO}_{2}\right)$ seen at exercise in patients with chronic heart failure. The inability to match regional lung perfusion to ventilation because of reduced cardiac output has been suggested as one of the limiting factors of exercise in chronic heart failure. To show ventilation to perfusion matching at rest and at peak exercise, eight male patients (mean age 66, range 52-73; mean left ventricular ejection fraction 30\%, range 16-45) in New York Heart Association class II underwent maximal symptom limited bicycle ergometry (starting at $50 \mathrm{~W}$, increasing by $10 \mathrm{~W} \mathrm{~min}^{-1}$ ). Minute ventilation, $\mathrm{VCO}_{2}$ and maximal oxygen consumption $\left(\mathrm{Vo}_{2 \max }\right)$ were measured from expired gas by mass spectrometry. $\mathrm{Vo}_{2 \max }$ under basal conditions ranged from 11.3 to $24 \cdot 1 \mathrm{ml} \cdot \mathrm{kg}^{-1} \cdot \mathrm{min}^{-1}$ (mean 14.6). On separate days, V/Q gamma camera imaging (inhaled krypton-81m gas/intravenous technetium-99m macroaggregated albumin) was performed at rest, and from $80 \%$ (previous peak exercise) heart rate during bicycle ergometry. Right lung vertical profiles (normalised to $100 \%=$ total lung radioactivity) were subtracted (V-Q) to estimate the vertical distribution of $\mathrm{V} / \mathrm{Q}$ mismatch. Summed differences (sign ignored) gave a numerical index of global mismatch. A V/Q mismatch was present at rest in all patients. The ability to reduce global $\mathrm{V} / \mathrm{Q}$ mismatch from rest to peak exercise correlated positively with $\mathrm{Vo}_{2 \max }$ on both treadmill exercise $(r=0.91, p<0.01)$ and bicycle ergometry $(\mathrm{r}=0.89, \mathrm{p}<0.01)$. The reduction in $\mathrm{V} / \mathrm{Q}$ mismatch also correlated with the mean arterial pressure at peak exercise $(\mathrm{r}=0.76, \mathrm{p}<0.05)$, but no correlation was shown with either peak systolic blood pressure, rate pressure product, change in mean arterial pressure from rest or resting left ventricular ejection fraction.

This suggests that the ability to match ventilation to perfusion regionally may be an important determinant of maximal exercise capacity.

\section{Platelet membrane glycoprotein (GPIIb-IIIa) expression and size are increased in acute myocardial infarction}

R E A Smith, H Giles, N Carden, J F Martin

Kings College School of Medicine, London; and The Wellcome Foundation Research Laboratories, Beckenham, Kent

Platelet aggregation, which plays a pivotal part in acute myocardial infarction (AMI), is mediated by the membrane glycoprotein (GP)IIb-IIIa. This study measured the relative number of GPIIb-IIIa complexes on platelets in AMI compared with control platelets. Blood was sampled within 24 hours of admission and AMI diagnosed by history, electrocardiography, and cardiac enzymes. The control group were admitted at the same time and AMI excluded. Platelet rich plasma was prepared from citrated samples and GPIIb-IIIa quantified using a specific fluorescent antibody and flow cytometry. To eliminate differences in the activation state of the platelets between the groups GPIIb-IIIa was measured before and after stimulation with $0.1 \mathrm{mM}$ adenoside diphosphate (ADP), or the thromboxane mimetic U46619 $(1.0 \mu \mathrm{M})$. There were, on average, $26 \%$ more GPIIb-IIIa complexes on platelets in AMI compared with controls (AMI $=23 \cdot 7(1 \cdot 7), \mathrm{n}=11$ and control $=18 \cdot 8(1 \cdot 3), n=10$, arbitrary units $p<0.05)$. Stimulation with ADP or U46619 significantly increased GPIIb-IIIa expression by a similar proportion (22-32\%) in each group. Expression of GPIIb-IIIa remained significantly greater in AMI platelets compared with controls ( $\mathrm{p}<0.05$ for both ADP and U46619 stimulated platelets). Forward scatter, measured as an index of platelet size, was $15 \%$ greater in AMI patients compared with controls $\quad(A M I=4.40 \quad(0.16), \quad$ control $=3.83 \quad(0.10)$, $\mathrm{p}<0.05$, arbitrary units).

Platelets in AMI have more GPIIb-IIIa complexes and are larger. As platelet size and protein content are determined at thrombopoiesis these platelets must have been circulating before AMI. Such platelets may be more reactive and, therefore, causally related to AMI.

Circadian variation in white cell aggregation and free radical markers in men with stable ischaemic heart disease

\section{A B Bridges, N A Scott, G P McNeill, T H Pringle, J J F Belch \\ Departments of Medicine and Cardiology, Ninewells Hospital, Dundee}

The fibrinolytic activity of blood has a circadian variation with increased thrombotic tendency in the morning. This may be a contributory factor to the circadian variation in the time of onset of thrombotic events. There has, however, recently been increasing interest into the role of the white blood cell (WBC) and free radicals (FRs) in thrombosis. We have previously reported a circadian variation in WBC aggregation and FR state in normal volunteers. No one has yet studied possible circadian variations in these parameters in patients with stable ischaemic heart disease. Ten men with stable ischaemic heart disease had blood samples collected at four hourly intervals from midday until midday the next day. The patients were ambulant until 00:00 at which time they went to bed and remained in bed until 08:00. The following were measured on each sample: WBC aggregation and malondialdehyde (MDA), which is a product of lipid peroxidation by FRs, the FR scavengers, plasma thiol (PSH), red blood cell glutathione, and superoxide dismutase, which are all altered in the presence of increased FR activity. Aggregation of WBC and PSH concentration had significant circadian variations $(p<0.015$ and $\mathrm{p}<0.001$ respectively). The WBC aggregation peak was at 12:00 and the trough at 08:00, the PSH peak was at 00:00, and the trough at 04:00. Concentration of MDA did not show any variation. The largest rise in WBC aggregation occurred from 08:00 to 12:00; such an increase in aggregation could predispose to microcirculatory occlusion. The concentration of the scavenger PSH was lowest at 04:00, at this time FR products as measured by MDA in the ischaemic heart disease patients did not fall, this is by contrast with normal volunteers.

This combination of low level of a plasma scavenger and a relatively raised $\mathrm{MDA}$ concentration may predispose to thrombosis at this time. 
Circadian variation in tissue plasminogen activator, its inhibitor, von Willebrand factor, and prostacyclin stimulating factor in men with stable ischaemic heart disease

\section{A B Bridges, M McLaren, T H Pringle, G P McNeill, J J F Belch \\ Departments of Medicine and Cardiology, Ninewells Hospital, Dundee}

A circadian variation in the fibrinolytic activity of blood has been reported in normal volunteers and patients with myocardial infarcts and unstable angina. This circadian variation in the thrombotic tendency is said to contribute to the increased incidence of thrombotic events in the morning. No one, however, has investigated circadian variations in tissue plasminogen activator antigen (tPA $\mathrm{Ag}$ ), plasminogen activator inhibitor (PAI), von Willebrand factor (fVIII vWFAg) or prostacyclin stimulating factor $\left(\mathrm{PGI}_{2} \mathrm{SF}\right)$ in patients with stable ischaemic heart disease. We therefore studied the circadian variation of tPA $\mathrm{Ag}, \mathrm{PAI}$, fVIII vWFAg, and $\mathrm{PGI}_{2}$ concentrations in 10 men with stable ischaemic heart disease on no regular drug treatment. Seven blood samples were collected at fourhourly intervals from 12:00 until the same time the next day. The subjects were ambulant from 08:10 until 00:00 at which time they went to bed. They remained in bed until 08:00 the next morning. Concentrations of PAI had a significant circadian variation $(p<0.001)$, peaks occurred at 04:00 and troughs at 20:00, no significant circadian variations were detected in fVIII vWFAg, or $\mathrm{PGI}_{2} \mathrm{SF}$ concentrations. These results show similar circadian patterns to those of our normal volunteers. Our data indicate, however, for the first time that a significant circadian variation of PAI concentrations exists in patients with stable ischaemic heart disease, which predisposes to thrombosis in the early morning hours.

Furthermore, interestingly, tPA Ag concentrations did not vary significantly during the study period, which is by contrast with the results obtained in normal volunteers. This observation suggests that patients with ischaemic heart disease are at risk early in the morning through both a rise in PAI concentrations and failure of increase in tPA Ag concentrations.

\section{Evidence of free radical production after successful thrombolytic therapy in acute myocardial infarction}

\section{J A Purvis, I S Young, E Trimble, A A Adgey Royal Victoria Hospital, Belfast}

In animal models, myocardial ischaemia and reperfusion are associated with free radical production although the relevance of such studies to the human myocardium is unclear. We have indirectly assessed free radical production after thrombolytic treatment for acute myocardial infarction. Sixty six patients seen within six hours of a myocardial infarction received two bolus injections of alteplase separated by 30 minutes. Coronary artery patency was assessed by angiography 90 minutes after the first bolus. Malondialdehyde (MDA), a marker of lipid peroxidation and the major lipid phase antioxidant vitamin $\mathrm{E}$, were measured at times $0,30,60$, and 90 minutes, six and 24 hours from $5 \mathrm{ml}$ of venous blood using high performance liquid chromatography. At 90 minutes, a statistically significant rise was seen in mean MDA concentrations compared with baseline $(0.91$ to 0.99 $\mu \mathrm{mol} / \mathrm{l} ; \mathrm{p}<0.05)$ and a significant fall was seen in mean vitamin $E$ concentration $(7 \cdot 15$ to $6.42 \mu \mathrm{mol} / \mathrm{mmol}$ cholesterol) among the 52 patients with thrombolysis in myocardial infarction trial (TIMI) 2 or 3 patency. These results are consistent with significant free radical generation. No significant change was detected amongst the 14 patients with TIMI 0 or 1 patency at 90 minutes.

The potential use of antioxidant agents to modify these changes should be investigated.

\section{Physical training improves skeletal muscle} metabolic abnormalities in patients with chronic heart failure: phosphorus-31 magnetic resonance spectroscopy study

S Adamopoulos, A J C Coats, L Arnolda, F Brunotte, C Thompson, T Meyer, J Stratton, G Radda, B Rajagopalan

Cardiac Department, John Radcliffe Hospital; and Medical Research Council NMR Unit, Oxford University

Muscle deconditioning has been considered one of the possible mechanisms for the intrinsic metabolic changes in skeletal muscle in chronic heart failure. To investigate the effects of physical training on skeletal muscle metabolism we studied 12 patients with ischaemic chronic heart failure (New York Heart Association II/III, ejection fraction 24 (3.4) $\%$, aged $62.4(2.6)$ ). Muscle $\mathrm{pH}$, inorganic phosphate $(\mathrm{Pi})$, phosphocreatine $(\mathrm{PCr})$ utilisation (expressed by the ratio $\mathrm{PCr} / \mathrm{PCr}+\mathrm{Pi}$ ), and adenosine diphosphate (ADP) concentrations were evaluated before and after eight weeks of home based bicycle exercise training $(20 \mathrm{~min} /$ day, six days/week) in a crossover randomised order. Serial ${ }^{31} \mathbf{P}$ spectra of the calf muscle were obtained at rest, throughout plantarflexion at incremental workloads until exhaustion and during the recovery period in both patients and a control group of five age matched healthy men. Training produced an increase in incremental plantarflexion exercise time $(9.4(8) v 7.4(7) \mathrm{min}, \mathrm{p}<0.005)$. Utilisation of $\mathrm{PCr}$ and concentrations of $\mathrm{ADP}$ during exercise were reduced significantly $(p<0.005$, ANOVA for repeated measures) by training, starting from the first minute $(0.81$ $(0.03) v 0.71(0.02)$ and $19.6(4.5) v 34.9(5.1)$ respectively), at all matched submaximal workloads and even at peak exercise $(0.59(0.05) v 0.48(0.05)$ and $30.8(4.3) v 51.1(7.8)$ respectively). The $\mathrm{PCr}$ and ADP recovery half times were significantly faster after training $(0.596(0.1) v 0.754(1)$, $\mathrm{p}<0.05$ and $0.3(0.04) \quad v 0.41(0.07) \mathrm{min}, \mathrm{p}<0.05$ respectively). Training produced no significant difference in plantarflexion induced changes in $\mathrm{pH}$. There were no significant differences in $\mathrm{PCr}$ and ADP concentrations between chronic heart failure patients after training and controls during the part of the plantarflexion exercise completed by all subjects. There was also no difference in PCr recovery half time between patients after training and controls.

Physical training can reverse the excessive $\mathrm{PCr}$ depletion and reduce $\mathrm{ADP}$ concentrations during exercise, and accelerate the rate of $\mathrm{PCr}$ resynthesis and ADP disappearance during recovery, indicating an improvement in the impaired oxidative metabolism of skeletal muscle seen in chronic heart failure consistent with increased mitochondrial content or improved function of the existing mitochondria. 